UPR-1249-T

\title{
F-Theory Compactifications with Multiple U(1)-Factors: Constructing Elliptic Fibrations with Rational Sections
}

\author{
Mirjam Cvetič ${ }^{1,2}$, Denis Klevers ${ }^{1}$ Hernan Piragua ${ }^{1}$ \\ ${ }^{1}$ Department of Physics and Astronomy, \\ University of Pennsylvania, Philadelphia, PA 19104-6396, USA \\ ${ }^{2}$ Center for Applied Mathematics and Theoretical Physics, \\ University of Maribor, Maribor, Slovenia
}

cvetic at cvetic.hep.upenn.edu, klevers at sas.upenn.edu, hpiragua at sas.upenn.edu,

\begin{abstract}
We study F-theory compactifications with $\mathrm{U}(1) \times \mathrm{U}(1)$ gauge symmetry on elliptically fibered Calabi-Yau manifolds with a rank two Mordell-Weil group. We find that the natural presentation of an elliptic curve $\mathcal{E}$ with two rational points and a zero point is the generic Calabi-Yau onefold in $d P_{2}$. We determine the birational map to its Tate and Weierstrass form and the coordinates of the two rational points in Weierstrass form. We discuss its resolved elliptic fibrations over a general base $B$ and classify them in the case of $B=\mathbb{P}^{2}$. A thorough analysis of the generic codimension two singularities of these elliptic Calabi-Yau manifolds is presented. This determines the general $\mathrm{U}(1) \times \mathrm{U}(1)$ charges of matter in corresponding F-theory compactifications. The matter multiplicities for the fibration over $\mathbb{P}^{2}$ are determined explicitly and shown to be consistent with anomaly cancellation. Explicit toric examples are constructed, both with $\mathrm{U}(1) \times \mathrm{U}(1)$ and $\mathrm{SU}(5) \times \mathrm{U}(1) \times \mathrm{U}(1)$ gauge symmetry. As a by-product, we prove the birational equivalence of the two elliptic fibrations with elliptic fibers in the two blow-ups $B l_{(1,0,0)} \mathbb{P}^{2}(1,2,3)$ and $B l_{(0,1,0)} \mathbb{P}^{2}(1,1,2)$ employing birational maps and extremal transitions.
\end{abstract}

March, 2013 


\section{Contents}

1 Introduction and Summary of Results 2

1.1 Summary of Results . . . . . . . . . . . . . . . . 3

2 Basics of Abelian Gauge Sectors in F-theory 7

2.1 Elliptic Curves with Multiple Rational Points . . . . . . . . . . . 7

2.2 Elliptic Calabi-Yau Manifolds with Rational Sections . . . . . . . . . . 8

2.3 Abelian Gauge Sectors in F-Theory . . . . . . . . . . . . . . . . . 11

3 Elliptic Fibrations with Two Rational Sections 12

3.1 Constructing an Elliptic Curve with two Rational Points . . . . . . . . . 13

3.2 Resolved Elliptic Curve in $d P_{2}$ and its Elliptic Fibrations . . . . . . . . 21

4 Matter Spectrum: Codimension Two Singularities 24

4.1 Factorized Weierstrass Form: charges $(1,0)$ and $(0,1) \ldots \ldots$

4.2 Doubly-Factorized Weierstrass Form: charge $(1,1) \ldots$. . . . . . . . 29

4.3 Singular Rational Sections: charges $(-1,1),(-1,-2),(0,2) \ldots \ldots$

4.4 Calculating Matter Multiplicities . . . . . . . . . . . . . 36

5 Anomaly Cancellation: a Consistency Check 39

6 Toric Elliptic Calabi-Yau Manifolds with Two

Rational Sections

6.1 Example 1.............................. 43

6.2 Example $2 \ldots \ldots \ldots \ldots \ldots$. . . . . . . . . . . . . . . . . . . . . . .

6.3 Example with $\mathrm{SU}(5) \ldots \ldots \ldots \ldots$. . . . . . . . . . . . . . . .

7 Equivalence of $B l_{(1,0,0)} \mathbb{P}^{2}(1,2,3)$ and $B l_{(0,1,0)} \mathbb{P}^{2}(1,1,2)$ Elliptic Fibrations 49

8 Outlook

A More Details on the Elliptic Curve in $d P_{2}$

B Nagell's Algorithm: Cubic to Weierstrass 58 


\section{Introduction and Summary of Results}

In recent years significant progress has been made on the construction of phenomenologically appealing models of particle physics from F-theory compactifications, initiated by the local model approach of [1, 2, 3, 4,. The road to the construction of semi-realistic compact F-theory GUTs models with $\mathrm{SU}(5)$ or $\mathrm{SO}(10)$ gauge group has been paved in [5, 6, 7, 8, 9], although a model passing most phenomenological tests has yet to be constructed. Our understanding of both the local approach as well its embedding into global compactifications has improved vastly over the last years [10, 11, 12]. Since the development of F-theory [13, 14, 15] the construction of non-Abelian gauge symmetry in F-theory compactifcations is well understood due to the full classification of codimension one singularities of elliptically fibered Calabi-Yau manifolds [16, 17, 18]. A recent reconsideration and careful analysis has closed remaining gaps in the understanding of singularities at higher codimension [19, 20, 21]. Constructive algorithms for the explicit construction of non-Abelian gauge symmetries in toric Calabi-Yau manifolds are developed [22, 23] and have recently been employed for an exhaustive exploration of $6 \mathrm{~d}$ gauge symmetries from F-theory on toric elliptic fibrations over $\mathbb{P}^{2}$ [24].

In contrast to this, the construction of Abelian gauge symmetries in F-theory is much less understood. One reason is the dependence of this question on the global geometry of the elliptic fibration. However, aspects of the physics of U(1)-symmetries can already be studied in local F-theory models employing spectral cover methods [25, 26, 6, 27, 28, 29, 30, 31. F-theory compactifications with an Abelian gauge theory sector arise from compactifications on elliptic Calabi-Yau manifolds $X$ with general fiber being an elliptic curve with rational points. Elliptic curves $\mathcal{E}$ with a so-called non-trivial Mordell-Weil group of rational points are a classical subject in mathematics [32, 33, 34, 35, 36]. These rational points lift to rational sections of the fibration $X$, that contribute new harmonic two-forms to the cohomology that support Abelian gauge fields in the F-theory effective action. The number of Abelian gauge fields is set by the rank of the Mordell-Weil group of the elliptic curve and its torsion subgroup gives rise to non-simply laced groups [15, 37, 38. Rank one Mordell-Weil groups in compact elliptic fibrations have been studied recently in the F-theory literature in varying contexts [39, 40, 41, 42, 43, 44, 45, 46].

The gauge theory arising from an F-theory compactification changes significantly in the presence of U(1)-gauge symmetries. Additional codimension two singularities support

singlet fields only charged under the U(1)-gauge fields and the charged matter fields of the non-Abelian sector split into multiple fields differing by their U(1)-charges. Furthermore, 
the structure of Yukawa-couplings are dominated by U(1)-selection rules. This additional structure has rich phenomenological implications in Beyond the Standard Model (BSM) model building, where it can be used to avoid dangerous proton decay operators, engineer realistic Yukawa textures or provide a solution to the $\mu$-problem [6, 27, 47, 39, 48, 30].

To broaden the applicability of F-theory to BSM physics it is desirable to construct Abelian sectors of higher rank in F-theory. For example, conservative SM-extensions by only a single $\mathrm{U}(1)$, e.g. in models $G_{S M} \times \mathrm{U}(1)$ with a $Z^{\prime}$-boson, have an Abelian sector $\mathrm{U}(1) \times \mathrm{U}(1)_{Y}$ of rank two and would require the understanding of rank two Mordell-Weil groups in F-theory. In addition, a classification of the possible Abelian gauge sector in F-theory analogous to the well-studied non-Abelian sector is lacking. However, see [49] for a systematic study of rational sections on toric K3-surfaces. We make some progress in this direction by constructing F-theory compactifications with a $\mathrm{U}(1) \times \mathrm{U}(1)$-sector ${ }^{1}$.

\section{$1.1 \quad$ Summary of Results}

In this work we construct for the first time an elliptic curve $\mathcal{E}$ and a resolved elliptic Calabi-Yau fibration $\hat{X}$ with Mordell-Weil (MW) group of rational sections of rank two. We systematically derive by purely algebraic methods the concrete presentation of the elliptic curve $\mathcal{E}$ as the Calabi-Yau onefold in the toric del Pezzo surface $d P_{2}^{2}$, An Ftheory compactification on $\hat{X}$ will have two $\mathrm{U}(1) \times \mathrm{U}(1)$-gauge symmetry and charged matter. We determine the full matter spectrum including matter multiplicities for the first time and show consistency with six-dimensional anomaly cancellation. The general procedure employed here can readily be generalized to higher rank MW-groups, and might be relevant for the classification of the possible Abelian sectors in F-theory.

It is the pivotal result of our analysis that it is necessary to depart from the Weierstrass and Tate form to study the curve $\mathcal{E}$ and its corresponding elliptic fibrations. The reasons for this can be summarized by the following key findings of our analysis:

i) Abandoning the paradigm of a holomorphic zero section: General models with two rational sections require that the zero section is only a rational, not a holomorphic section.

ii) Departure from Tate/Weierstrass form: The zero section in the Tate or Weierstrass model is always holomorphic. Thus we have to work in a realization of elliptic fibrations that naturally incorporate a rational zero section such as toric geometry.

\footnotetext{
${ }^{1}$ For a study of Tate models with multiple rational points at $z=0$ see 45 .

${ }^{2}$ We note that a model with elliptic fiber in $d P_{1}$ has been studied in [46].
} 
iii) Toric elliptic curves: Toric geometry greatly facilitates the construction of some higher rank MW-groups. Rational sections are directly realized as toric divisor ${ }^{3}$.

iv) Birational maps: Due to i) the map of the toric elliptic curve iii) with rational zero section to the Weierstrass model ii) with holomorphic zero section is rational.

We emphasize that it is also imperative to present $\mathcal{E}$ as the hypersurface in $d P_{2}$ because the Weierstrass model of the elliptic fibration $\hat{X}$ will be generically singular. The birational map from the Weierstrass form to $d P_{2}$ will automatically resolve all singularities in any codimension, which is in accord with the smoothness of generic toric Calabi-Yau hypersurfaces [50]. Thus, the understanding of the rational map involved is also the key to analyze higher codimension singularities which determine the F-theory matter spectrum.

In the following we summarize the key points of our program leading to the construction of the curve $\mathcal{E}$ in $d P_{2}$ and the analysis of the associated elliptic fibrations $\hat{X}$, its rational sections, its Tate $\AA^{4}$ and Weierstrass form and codimension two singularities.

- We first derive the representation of an elliptic curve $\mathcal{E}$ over a field $K$ with two rational points $Q, R$ and a zero point $P$. To this end we consider a degree three line bundle $M=\mathcal{O}(P+Q+R)$ with holomorphic sections $u, v$ and $w$ and find a cubic relation among these holomorphic sections. This naturally leads to an embedding of the elliptic curve $\mathcal{E}$ as the non-generic cubic curve in $\mathbb{P}^{2}$, which is resolved into the Calabi-Yau onefold in the del Pezzo surface $d P_{2}$. We determine the birational map of this elliptic curve in $d P_{2}$ to its Tate form and Weierstrass form with respect to the zero point $P$. This birational map allows us to determine the coordinates of the rational points $Q$ and $R$ in Weierstrass form. Then we construct an elliptically fibered Calabi-Yau threefold $\mathcal{E} \rightarrow \hat{X} \rightarrow B$ over a base $B$ with general fiber being the elliptic curve $\mathcal{E}$ in $d P_{2}$. The point $P$ becomes the zero section $\hat{s}_{P}$ of the fibration and the rational points $Q, R$ lift to rational sections $\hat{s}_{R}, \hat{s}_{Q}$.

- We find the matter content of an F-theory compactification on $\hat{X}$ by analyzing the codimension two singularities of the elliptic fibration, by deriving the Weierstrass representation of the fibration and the rational sections. The resolved fiber in $d P_{2}$ over all codimension two loci is a reducible $I_{2}$ curve, i.e. the elliptic curve $\mathcal{E}$ splits into two rational curves $\mathcal{E}=c_{1}+c_{2}$ intersecting in two points. This can be viewed

\footnotetext{
${ }^{3}$ This fact has also been exploited in [49, 43, 45, 46].

${ }^{4}$ As will be evident from the concrete exposition in section 3.1. a global Tate model might not exist for all elliptic fibrations.
} 
as the Dynkin diagram of an extended SU(2) group arising in codimension two. The behavior of the rational sections is crucial for the analysis of matter. In the generic elliptic fibration with our $d P_{2}$-fiber we identify six different codimension two loci. Along three loci one of the sections $\hat{s}_{P}, \hat{s}_{Q}$ and $\hat{s}_{R}$ are ill-behaved and we have to resolve $\hat{X}$ in the base $B$ along the corresponding loci. In the resolved space the section wraps an entire fiber component. Physically, these loci are distinguished as the loci of matter of more exotic $\mathrm{U}(1) \times \mathrm{U}(1)$-charges with charges $(-1,1),(-1,-2)$ and $(0,2)$. Further matter is located whenever one of our two rational sections $\hat{s}_{Q}$, $\hat{s}_{R}$ intersect the zero section $\hat{s}_{P}$. These degenerations contribute matter of charges $(1,0)$ and $(0,1)$ depending on which section touches the zero section. Finally, a third type of singularity arises when $\hat{s}_{Q}$ and $\hat{s}_{R}$ coincide and touch the zero section $\hat{s}_{R}$. This is still a codimension two phenomenon leading to matter of charge $(1,1)$.

- Finally, we determine the multiplicities of matter fields. We demonstrate that this counting is comparably involved since the various codimension two loci corresponding to different matter fields intersect. Concretely, assuming matter of charges $(-1,1),(-1,-2)$ and $(0,2)$ is located at a collection of points $\operatorname{loc}_{1}$ and matter with charges $(1,0),(0,1)$ and $(1,1)$ is located at points $l o c_{2}$, we find that $l o c_{2}$ is automatically obeyed at $l o c_{1}$, i.e. $l o c_{1} \cap l o c_{2} \neq 0$. The appropriate multiplicity of the latter matter fields at $l o c_{2}$ is then determined by subtracting the multiplicities of the former matter fields at $l o c_{1}$, taking into account the order of vanishing of $l o c_{2}$ along $l o c_{1}$. This complication also arose in [43]. We show that the appropriate treatment involves the application of the resultant of $l o c_{2}$ with $l o c_{1}$ as its root.

We would also like to emphasize that we find all six-dimensional anomalies to be canceled for the matter charges and multiplicities found. This gives us some evidence for the completeness of our analysis of codimension two singularities of the fibration $\hat{X}$. Since the matter content is a codimension two phenomenon, we expect our results for the matter charges to hold in general, in particular for Calabi-Yau fourfolds.

We construct three global models as toric Calabi-Yau hypersurfaces with base $\mathbb{P}^{2}$. We present two different models with $\mathrm{U}(1) \times \mathrm{U}(1)$ gauge group, that differ in the complexity of the behavior of the rational sections $\hat{s}_{Q}, \hat{s}_{R}$. In the first model both sections never intersect the zero sections and only charge $(1,0),(0,1),(1,1)$ matter is realized. In the second model we allow for a non-trivial behavior of the section $\hat{s}_{R}$ and confirm the existence of all six different matter multiplets. In both cases we confirm by anomaly cancellation the consistency and completeness of our analysis. We explicitly write down 
the $4 \mathrm{~d}$ polytope realizing a model with $\mathrm{SU}(5) \times \mathrm{U}(1)^{2}$ gauge group. We observe that matter in the $\mathbf{5}$-representation splits into five matter representation $\boldsymbol{5}_{\left(q_{1}, q_{2}\right)}$ differing by their $\mathrm{U}(1)^{2}$-charges. The $\mathbf{1 0}$ matter curves does not split.

Finally we exploit the use of birational geometry to study extremal transitions between elliptic fibrations of two different fiber types [51] yielding two equivalent, but different descriptions of the same F-theory vacua with one $\mathrm{U}(1)$. We perform the extremal transition from the elliptic curve in $\mathbb{P}^{2}(1,2,3)$, which is the standard Tate form, to the curve in $B l_{(1,0,0)} \mathbb{P}^{2}(1,2,3)$, which is still in Tate form, but with Tate coefficient $a_{6} \equiv 0$. Ultimately, simple toric techniques allow us to readily obtain the birational map from the latter model to the resolved quartic curve in $B l_{(0,1,0)} \mathbb{P}^{2}(1,1,2)$, which immediately reproduces the map found in [43]. Extending this birational map to elliptic fibrations, this thereby establishes the birational equivalence of the U(1)-restricted Tate-model [39] and the fibration with elliptic curve in $B l_{(0,1,0)} \mathbb{P}^{2}(1,1,2)$. We emphasize, however, that this equivalence only holds for elliptic fibrations of a certain class.

This paper is organized as follows. In section 2 we introduce the basic concepts and constructions for elliptic fibrations with a Mordell-Weil group for F-theory compactifications with an Abelian gauge theory sector. In section 3 we derive the form of the general elliptic Calabi-Yau manifold $\hat{X}$ with two rational sections. For this purpose we first derive the presentation of an elliptic curve $\mathcal{E}$ over a field $K$ with two rational points as the hypersurface in $d P_{2}$ and then readily generalize to Calabi-Yau elliptic fibrations, that we classify for base $B=\mathbb{P}^{2}$. We elucidate the structure of the resolved geometry of the elliptic curve $\mathcal{E}$ in $d P_{2}$. In section 4 we study in detail the general matter spectrum of the F-theory compactification on the resolved threefold $\hat{X}$. We show that both its singular Weierstrass model as well as the resolution to $\hat{X}$ with $d P_{2}$-fiber are crucial to understand the structure of all codimension two singularities. We determine both $\mathrm{U}(1) \times \mathrm{U}(1)$-charges and matter multiplicities. In section 5 we check that our spectrum is in general consistent with anomaly cancellation in $6 \mathrm{~d}$. We present three concrete toric realizations of elliptic threefolds $\hat{X}$ over $B=\mathbb{P}^{2}$ in section 6 . Two examples have $\mathrm{U}(1) \times \mathrm{U}(1)$ gauge symmetry whereas we add an SU(5)-GUT sector in a third example. We conclude by an application of birational maps in section 7 to show the equivalence of elliptic fibrations with fibers of two different types, both of which with rank one MW-group. Section 8 contains our outlook. We have two appendices $\mathrm{A}$ and $\mathrm{B}$ with details on the Weierstrass model of the elliptic curve $\mathcal{E}$ and Nagell's algorithm of the cubic.

While we were preparing this manuscript the paper [52] appeared which has some overlap with our discussion. 


\section{Basics of Abelian Gauge Sectors in F-theory}

In this section we review the notion of the Mordell-Weil group as the group of rational points on an elliptic curve in section 2.1. Then we discuss the geometry of Calabi-Yau manifolds formed as elliptic fibrations with general fiber given by such an elliptic curve in section 2.2. The rational points are lifted to rational sections of the elliptic fibration and contribute additional cycles to the homology group of these manifolds. The Abelian sector of an F-theory compactification on these elliptically fibered Calabi-Yau manifolds, as explained in section 2.3, is determined by the structure of these rational sections. The reader interested in the results of our analysis can skip this section and directly proceed with section 3.1.

\subsection{Elliptic Curves with Multiple Rational Points}

To set the stage for our later discussion, we begin with the most concrete definition of an elliptic curve $\mathcal{E}$ over a field $K$ in terms of a Weierstrass model. The Tate form of the Weierstrass model reads

$$
y^{2}+a_{1} x y z+a_{3} y z^{3}=x^{3}+a_{2} x^{2} z^{2}+a_{4} x z^{4}+a_{6} z^{6}
$$

with $a_{i}$ denoting numbers in a field $K$. In elliptic fibrations of $\mathcal{E}$ over a base $B, K$ will be identified with the function field of $B$. In the chosen projectivization (2.1) is a one-dimensional Calabi-Yau hypersurface in the weighted projective space $\mathbb{P}^{2}(1,2,3)$ with homogeneous coordinates $[z: x: y]$. The Tate form can be brought into the reduced Weierstrass form

$$
\tilde{y}^{2}=\tilde{x}^{3}+f \tilde{x} z^{4}+g z^{6}
$$

by the variable transformation

$$
\tilde{x}=x+\frac{1}{12} b_{2} z^{2}, \quad \tilde{y}=y+\frac{1}{2} a_{1} x z+\frac{1}{2} a_{3} z^{3}
$$

with the following definitions

$$
\begin{aligned}
f & =-\frac{1}{48}\left(b_{2}^{2}-24 b_{4}\right), \quad g=-\frac{1}{864}\left(-b_{2}^{3}+36 b_{2} b_{4}-216 b_{6}\right) \\
b_{2} & =a_{1}^{2}+4 a_{2}, \quad b_{4}=a_{1} a_{3}+2 a_{4}, \quad b_{6}=a_{3}^{2}+4 a_{6} \\
\Delta & =-16\left(4 f^{3}+27 g^{2}\right)=-8 b_{4}^{3}+\frac{1}{4} b_{2}^{2} b_{4}^{2}+9 b_{2} b_{4} b_{6}-\frac{1}{4} b_{6} b_{2}^{3}-27 b_{6} .
\end{aligned}
$$


Here the quantity $\Delta$ defined in the last line of $(2.4)$ is the discriminant of the Weierstrass equation (2.2). If $\Delta=0$ then the elliptic curve defined by (2.2) is singular.

Both the Tate form 2.1) as well as the reduced Weierstrass form 2.2 have one distinguished point $P$ at $[z: x: y]=[0: 1: 1]$, referred to as the zero point. In general, a rational point on the elliptic curve $\mathcal{E}$ is defined as a point with coordinates in the designated field $K$. The set of rational points on $\mathcal{E}$ form an Abelian group under addition that is naturally defined in the Weierstrass form (2.2). The group of rational points, with $P$ as the zero, is the Mordell-Weil group of the curve $\mathcal{E}$. The Mordell-Weil group is finitely generated, i.e. it is the sum of a torsion subgroup and $\mathbb{Z}^{r}$, and $r$ is the Mordell-Weil rank. We introduce a basis of the torsionless subgroup of the Mordell-Weil group as $Q_{m}, m=1, \ldots, r$.

We note that the presence of a rational point can imply a factorization of the Weierstrass form. A case of particular interest later in this work is a point $Q$ of the form $[z: \tilde{x}: \tilde{y}]=[1: A: B]$ for given numbers $A, B$ in $K$. In this case, the Weierstrass equation (2.2) factorizes as

$$
\left(\tilde{y}-B z^{3}\right)\left(\tilde{y}+B z^{3}\right)=\left(\tilde{x}-A z^{2}\right)\left(\tilde{x}^{2}+A \tilde{x} z^{2}+C z^{4}\right),
$$

as is easily checked by plugging in the point $Q$. This implies that the coefficients $f, g$ in (2.2) as well as the discriminant in (2.4) can be parametrized as

$$
f=C-A^{2}, \quad g=B^{2}-A C, \quad \Delta=16\left(27 B^{2}\left(2 A C-B^{2}\right)+\left(A^{2}-4 C\right)\left(2 A^{2}+C\right)^{2}\right)
$$

This parametrization will prove useful in the discussion of matter with only charge 1 under the Abelian gauge field corresponding to the rational point $Q$ [43], i.e. codimension two singularities only due to the presence of the point $Q$ in the fiber $\mathcal{E}$ alone. We add that factorization properties of the Tate form (2.1) due to rational points of the form $z=0$ have been discussed in [45].

\subsection{Elliptic Calabi-Yau Manifolds with Rational Sections}

In this section we briefly discuss the geometry of an elliptically fibered Calabi-Yau threefold $X$, although we note that the following holds for general complex dimension of $X$.

By definition an elliptic fibration over a base $B$ is defined by a holomorphic projection $\pi: X \rightarrow B$ to the base $B$. We will be interested in fibrations with general fiber $\mathcal{E}=\pi^{-1}(p t)$ over a generic point $p t$ in $B$ given by elliptic curve with a zero point and 
a number of rational points. As mentioned before we can always describe an elliptic fibration over $B$ by its Weierstrass model (2.2), where the field $K$ is replaced by the function field of $B$. Concretely, by the Calabi-Yau condition $f, g$ are sections of the line bundles $K_{B}^{-4}$ respectively $K_{B}^{-6}$, where $K_{B}$ denotes the canonical bundle of the base. If it exists globally we can also construct the Tate form ${ }^{5}$ (2.1) where the coefficients $a_{i}$ take values in $K_{B}^{-i}$. The holomorphic zero section in the Tate and Weierstrass form is given by $z=0$.

However, when having global questions in mind, such as the global resolution of singularities of the elliptic fibration $X$ or the construction of rational sections, it is of advantage to consider elliptic fibrations $X$ with the general elliptic fiber $\mathcal{E}$ given as the Calabi-Yau hypersurface in one of the 16 two-dimensional toric varieties. It is always possible, as discussed at the end of section 2.1, to obtain the Weierstrass form of these fibrations by a birational map. In this note we will focus on the elliptic fibration with general fiber in $d P_{2}$, cf. section 3.1.

When we fiber an elliptic curve $\mathcal{E}$ over a base $B$ its zero point $P$ becomes the zero section $\hat{s}_{P}$ and its rational points $Q_{m}$ lift to rational sections $\hat{s}_{m} \equiv \hat{s}_{Q_{m}}$ of the elliptic fibration $\pi: X \rightarrow B$. All of these sections define injective maps $\hat{s}_{P}, \hat{s}_{m}: B \hookrightarrow X$ and the group generated by the $\hat{s}_{m}$ is the Mordell-Weil group of the elliptic fibration $X$. A holomorphic section, that is in the literature typically denoted by $\sigma$, defines a holomorphic injection $\sigma: B \hookrightarrow X$ on all of $B$. However, a rational section does in general not vary holomorphically over the base $B$. Indeed, over codimension two or higher the rational section can be ill-defined and wrap components of the reducible fiber over the singular loci [43, 46]. Thus, rational sections $\hat{s}_{m}$ can only be defined on the blow-up $\pi_{B}: \hat{B} \rightarrow B$ of $B$ along the singular loci of $\hat{s}_{m}$ with $\pi_{B}$ denoting the blow-down map. Consequently, a rational section defines only a birational map $\hat{s}_{m}: \hat{B} \rightarrow B \hookrightarrow X$ of the base $B$ into $X$.

In sections 3.1 and 4, we study elliptic fibrations with general fiber given by an elliptic curve $\mathcal{E}$ with two rational points $Q$, and $R$ and a zero section $P$. As we see there, in these cases even the zero section $\hat{s}_{P}$ is not defined over codimension two and wraps fiber components of the elliptic fiber. A Calabi-Yau elliptic fibration without a holomorphic zero section still defines a valid F-theory background, although these most general fibrations have only recently drawn attention in the F-theory literature. As we discuss in section 4 , the behavior of the three rational sections $\hat{s}_{P}$ and $\hat{s}_{Q}, \hat{s}_{R}$ leads to a rich structure of charged matter.

\footnotetext{
${ }^{5}$ See $[53$ for a reconsideration of the validity of the Tate model in global F-theory compactifications.
} 
The group of divisors, or its dual group $H^{(1,1)}(\hat{X})$, on the smooth elliptic Calabi-Yau manifold $\hat{X} \rightarrow X$ arising from $X$ by resolving all singularities, is generated by divisors $D_{A}$ that fall into four different classes of divisors:

- the zero section $\hat{s}_{P}$ of the fibration with homology class $S_{P}$, which in the case of a holomorphic section $\sigma$ agrees with the class of the base $B$,

- the rational sections $\hat{s}_{m}, m=1, \ldots, r$, with divisor classes $S_{m}$ generating the Mordell-Weil group of rational sections of the elliptic fibration of $X$,

- the vertical divisors $D_{\alpha}=\pi^{*}\left(D_{\alpha}^{\mathrm{b}}\right), \alpha=1, \ldots, h^{(1,1)}(B)$, of the fibration that are inherited from divisors $D_{\alpha}^{b}$ in the base $B$,

- exceptional divisors $D_{i_{I}}$ resolving singularities of $X$ from singularities in its elliptic fibration at irreducible components $\Delta_{I}=0$ of the discriminant locus $\Delta=0$ in $B$.

We summarize this basis of divisors on $\hat{X}$ as

$$
D_{A}=\left(B, S_{m}, D_{\alpha}, D_{i}\right), \quad A=0,1, \ldots, h^{(1,1)}(\hat{X}) .
$$

We conclude with some key intersection properties of the divisors $D_{A}$. The exceptional divisors $D_{i_{I}}$ over one common discriminant locus intersect as

$$
D_{i_{I}} \cdot D_{i_{J}} \cdot D_{\alpha}=-C_{i_{I} j_{I}}^{(I)} S_{(I)} \cdot B \cdot D_{\alpha}
$$

where $C_{i_{I} j_{I}}^{(I)}$ denotes the Cartan matrix of an ADE-group $G_{(I)}$ in case of an ADEsingularity in the fibration. In this case we denote the $D_{i_{I}}$ as the Cartan divisors of the corresponding ADE group. Here the divisors $S_{(I)}=\pi^{*}\left(S_{(I)}^{\mathrm{b}}\right)$ are related to the components of the discriminant and the $S_{(I)}^{\mathrm{b}}$ are the loci in the base $B$ wrapped by 7-branes supporting a gauge group $G_{(I)}$ in F-theory. Upon intersecting the Cartan divisors $D_{i_{I}}$ with a divisor $\tilde{D}$ in $B$ that intersects $S_{(I)}$ in a point, we obtain a rational curve that is localized over $S_{(I)}$. The relation (2.8) teaches us that this curve is to be identified with minus the root $\alpha_{i_{I}}$ of the Lie-algebra of the ADE-group under consideration, i.e.

$$
\mathcal{C}_{-\alpha_{i_{I}}}:=D_{i_{I}} \cdot \tilde{D} \quad \text { for } \quad \tilde{D} \cdot S_{(I)} \cdot B=1 .
$$

Next we turn to the divisors $S_{P}$ and $S_{m}$ of the sections. By construction the divisors corresponding to a section will intersect the general fiber $F \cong \mathcal{E}$ as

$$
S_{P} \cdot F=S_{m} \cdot F=1 .
$$

In addition we note that any section of $X$ also has to obey [43]

$$
B^{2}=-\left[c_{1}(B)\right] \cdot B, \quad S_{m}^{2} \cdot D_{\alpha}=-\left[c_{1}(B)\right] \cdot S_{m} \cdot D_{\alpha},
$$

where the first relation holds in homology and $c_{1}(B)$ is the first Chern class of the base. 


\subsection{Abelian Gauge Sectors in F-Theory}

In an F-theory compactification on $X$ gauge fields $A^{a}$ arise by expanding the M-theory three-form $C_{3}$ in the dual M-theory compactification ${ }^{6}$ along appropriate $(1,1)$-forms $\omega_{a}$,

$$
C_{3}=\sum_{a} A^{a} \omega_{a} .
$$

These are gauge fields along the Cartan generators of all $G_{(I)}$ and along U(1) groups. The U(1)-gauge fields correspond to rational sections $\hat{s}_{m}$ and are constructed from the cohomology classes dual to the divisors $S_{m}$ of the rational sections by the Shioda map.

The Shioda map roughly speaking describes an orthogonalization procedure in the (co)homology group of $\hat{X}$. It is defined as the map from the Mordell-Weil group to $H^{(1,1)}(\hat{X})$ given by [43, 55, 44]

$$
\sigma\left(\hat{s}_{m}\right):=S_{m}-\tilde{S}_{P}-\left(S_{m} \cdot \tilde{S}_{P} \cdot D_{\alpha}\right) \eta^{\alpha \beta} D_{\beta}+\sum_{I}\left(S_{m} \cdot \mathcal{C}_{-\alpha_{i_{I}}}\right)\left(C_{(I)}^{-1}\right)^{i_{I} j_{I}} D_{j_{I}},
$$

where the curves $\mathcal{C}_{-\alpha}$ have been defined in 2.9$)$ and $C_{(I)}^{-1}$ is the inverse of the Cartan matrix determined in (2.8). The divisor $\tilde{S}_{P}=S_{P}+\frac{1}{2} \pi^{*} c_{1}(B)$ has been introduced for convenience, and is of relevance for the match of the F- and M-theory dual effective actions [56, 57]. The matrix $\eta^{\alpha \beta}$ is the inverse of the intersection form of the divisors on the base,

$$
\eta_{\alpha \beta}=D_{\alpha}^{\mathrm{b}} \cdot D_{\beta}^{\mathrm{b}} .
$$

The Shioda map (2.13) maps into the orthogonal complement $7^{7}$ in $H^{(1,1)}(\hat{X})$ generated by the zero section $S_{P}$, the vertical divisors $D_{\alpha}$ and the Cartan divisors $D_{i_{I}}$. Thus it ensures that the gauge field associated to $\sigma\left(\hat{s}_{m}\right)$ by the reduction (2.12) along the $(1,1)$-form $\omega_{m}$ defined as the Poincare dual of $\sigma\left(\hat{s}_{m}\right)$ is a U(1)-gauge field.

Having defined the Shioda map (2.13) it is straightforward to calculate U(1)-charges of matter fields in F-theory. The matter fields arise from M2-branes wrapping rational curves $c$ in the fiber of the elliptic fibration that are localized in codimension two in the base $B$. Under the assumption of a holomorpic zero section with $S_{P}=B$, the charge of the M2-brane state under the $\mathrm{U}(1)$-gauge field corresponding to the rational section $\sigma_{m}$ is then calculated as [43]

$$
\sigma\left(\hat{s}_{m}\right) \cdot c=\left(S_{m} \cdot c\right)+\sum_{I}\left(S_{m} \cdot \mathcal{C}_{-\alpha_{i_{I}}}\right)\left(C_{(I)}^{-1}\right)^{i_{I} j_{I}}\left(D_{j_{I}} \cdot c\right) .
$$

\footnotetext{
${ }^{6}$ See [54] for a general discussion of this and a derivation of the full F-theory effective action.

${ }^{7}$ The inner product is the Néron-Tate height pairing [35.
} 
This follows readily using the definition of the Shioda map (2.13) and the fact, that the isolated curves $c$ neither intersect vertical divisors nor the holomorphic zero section.

We note that the intersection matrix between the $\sigma\left(\hat{s}_{m}\right)$ projected into the homology of the base by $\pi: \hat{X} \rightarrow B$ is $\pi\left(\sigma\left(\hat{s}_{m}\right) \cdot \sigma\left(\hat{s}_{n}\right)\right)=\pi\left(S_{m} \cdot S_{n}\right)+\left[K_{B}\right]-\pi\left(S_{m} \cdot B\right)-\pi\left(S_{n} \cdot B\right)+\left(\mathcal{C}_{(I)}^{-1}\right)^{i_{I} j_{I}}\left(S_{m} \cdot \mathcal{C}_{-\alpha_{i_{I}}}\right)\left(S_{n} \cdot \mathcal{C}_{-\alpha_{j_{I}}}\right) S_{(I)}^{\mathrm{b}}$.

Here, we introduced the definition

$$
\pi(\mathcal{C})=\left(\mathcal{C} \cdot D_{\alpha}\right) \eta^{\alpha \beta} D_{\alpha}^{\mathrm{b}}
$$

of the Néron-Tate height pairing for a curve $\mathcal{C}$ and employed the normalized coroot matrix of the $I$-th 7 -brane gauge group

$$
\mathcal{C}_{i_{I} j_{I}}^{(I)}=\frac{2}{\lambda_{I}\left\langle\alpha_{i_{I}}, \alpha_{j_{I}}\right\rangle} C_{i_{I} j_{I}}^{(I)} .
$$

In this expression we denote the inner product on the Lie-algebra by $\langle\cdot, \cdot\rangle$ and introduced the length squared $\lambda_{I}=\frac{2}{\left\langle\alpha_{0}, \alpha_{0}\right\rangle}$ of the maximal root $\alpha_{0}$. The intersections (2.16) will be relevant for the discussion of anomaly cancellation in section 5 .

We conclude by noting that if the zero section $\hat{s}_{P}$ is only rational, the formula for the $\mathrm{U}(1)$-charge has to be modified. The reason for this is that we have to relax the condition $B \cdot c=0$ and in general assume a non-trivial intersection

$$
S_{P} \cdot c \neq 0,
$$

where $S_{P}$ denotes the homology class of the rational zero section. Then 2.15 has to be replaced by

$$
\sigma\left(\hat{s}_{m}\right) \cdot c=\left(S_{m} \cdot c\right)-\left(S_{P} \cdot c\right)+\sum_{I}\left(S_{m} \cdot \mathcal{C}_{-\alpha_{i_{I}}}\right)\left(C_{(I)}^{-1}\right)^{i_{I} j_{I}}\left(D_{j_{I}} \cdot c\right) .
$$

In contrast, the formula $(2.16)$ for the intersections of sections does not have to be modified. We note that (2.20) has also been used in [46].

\section{Elliptic Fibrations with Two Rational Sections}

In this section we determine an elliptic fibration with two rational sections and a zero section, i.e. an elliptic curve with Mordell-Weil group of rank two. This curve serves as 
the model for the general elliptic fiber in an elliptically fibered Calabi-Yau manifold. As discussed in section 2 the F-theory compactification on such a Calabi-Yau admits two $\mathrm{U}(1)$-gauge groups.

We first find in section 3.1 that any elliptic curve $\mathcal{E}$ with three marked points (two rational and the zero point) has a representation as a non-generic cubic in $\mathbb{P}^{2}$. We argue further that this elliptic curve should be properly viewed as the generic CalabiYau onefold in the del Pezzo surface $d P_{2}$, which is the blow up of $\mathbb{P}^{2}$ at two generic points. However, we first focus on the singular model of $\mathcal{E}$ by neglecting the blow-ups in $\mathbb{P}^{2}$. This allows us to compute the Weierstrass model for $\mathcal{E}$ with respect to one of the three rational points on it and derive the location of the two other rational points in Weierstrass coordinates.

Then, in section 3.2, we discuss the resolution geometry of $\mathcal{E}$ in $d P_{2}$ that we obtain by performing the two blow-ups in $\mathbb{P}^{2}$. The understanding of the resolved geometry is relevant to resolve elliptic fibrations with general fiber being the curve $\mathcal{E}$. We conclude by constructing elliptic fibrations with $\mathcal{E}$ and find that all elliptic fibrations with base $B=\mathbb{P}^{2}$ are classified by two integers $n_{2}$ and $n_{12}$. We will consequently denote the families of $d P_{2}$-fibrations over $\mathbb{P}^{2}$ we have found as $d P_{2}\left(n_{2}, n_{12}\right)$.

Our discussion partly follows and extends the techniques of appendix B of [43].

\subsection{Constructing an Elliptic Curve with two Rational Points}

Our discussion in this section is based on the following basic mathematical fact: Given an algebraic variety $\mathrm{X}$ with a very ample line bundle $8 M$ defined over it, we can find an embedding of $X$ into projective space. If this is the case, we will have enough independent global sections $a_{0}, \cdots, a_{n}$ such that for every point $x \in X$ there exists at least one section not vanishing at this point. Thus, there is an immersion

$$
f: \quad X \rightarrow \mathbb{P}^{n} \quad x \mapsto\left[a_{0}(x): \cdots: a_{n}(x)\right]
$$

with $M \cong f^{*}(\mathcal{O}(1))$.

The algebraic variety we are interested in is an elliptic curve $\mathcal{E}$ over a field $K$. We find the embedding of the curve $\mathcal{E}$ into projective space, or more generally a toric variety, explicitly as a hypersurface. For this purpose we consider the global sections of powers

\footnotetext{
${ }^{8} \mathrm{~A}$ very ample line bundle is a line bundle that has "enough" global sections so that its base variety is embedable into projective space.
} 
of $M^{k}$. For sufficiently large $k$, in our case $k=3$, not all sections are independent and the relation between them yield the desired hypersurface equation.

As a warm-up we use this logic to derive the Tate form (2.1) of an elliptic curve $\mathcal{E}$ with only the zero point $P$ as follows. We introduce the degree one line bundle $M=\mathcal{O}(P)$ over $\mathcal{E}$ and consider the homogeneous coordinates $[z: x: y]$ on $\mathbb{P}^{2}(1,2,3)$ as sections of the line bundles $M, M^{2}$ and $M^{3}$, respectively. We recall that in the case of an elliptic curve $\mathcal{E}$ the Riemann-Roch theorem tells us that the number of independent global holomorphic sections of a line bundle $M$ of degree $d$ is $h^{0}(X, M)=d$. Then the bundle $M^{6}$ has six independent holomorphic sections, however, we can construct seven sections from $z, x$, $y$. Thus, there has to be a relation between these sections which yields precisely the Tate form 2.1$)$ in $\mathbb{P}^{2}(1,2,3)$.

The same strategy applies to finding the equation that describes an elliptic curve with rational points $Q_{m}$. Concretely, we first find the sections $[z: x: y]$ of $\mathcal{O}(k P)$, $k=1,2,3$, and then determine the relation between sections of higher degree generated from $[z: x: y]$. We perform this procedure in the following for an elliptic curve $\mathcal{E}$ with a zero point $P$ and two rational points denoted $Q$ and $R$. In the same spirit as before we first start with a general line bundle $M$ of degree three that we then specialize to $M=\mathcal{O}(P+Q+R)$. The group of holomorphic section $H^{0}(M)$ is generated by three sections denoted $u, v, w$. The space $H^{0}(2 M)$ has dimension six with sections $u^{2}, v^{2}, w^{2}$, $u v, v w$ and $w u$. The space $H^{0}(3 M)$ must have nine independent sections, however we know ten of them. Consequently, there has to be a linear relation of the form

$$
s_{1} u^{3}+s_{2} u^{2} v+s_{3} u v^{2}+s_{4} v^{3}+s_{5} u^{2} w+s_{6} u v w+s_{7} v^{2} w+s_{8} u w^{2}+s_{9} v w^{2}+s_{10} w^{3}=0,
$$

with coefficients $s_{i}$ in the field $K$. This equation can be interpreted as the cubic hypersurface in $\mathbb{P}^{2}$. Even more it is a section of its anti-canonical bundle $\mathcal{O}(3 H)$, see figure 1. where $H$ denotes the hyperplane class in $\mathbb{P}^{2}$. Thus the zero of this section defines the one-dimensional Calabi-Yau manifold, i.e. the torus?

Now we specialize to $M=\mathcal{O}(P+Q+R)$. Let us assume that the section $u$ vanishes at the three points $P, Q$ and $R$, then equation $(3.2)$ simplifies to

$$
s_{4} v^{3}+s_{7} v^{2} w+s_{9} v w^{2}+s_{10} w^{3}=0 .
$$

Performing appropriate shifts of the coordinates $v$ and $w$ we can always get rid of the coefficients $s_{4}$ and $s_{10}$. However, these variable transformations involve square roots of

\footnotetext{
${ }^{9}$ There is a one-to-one correspondence between elliptic curves and two-tori.
} 

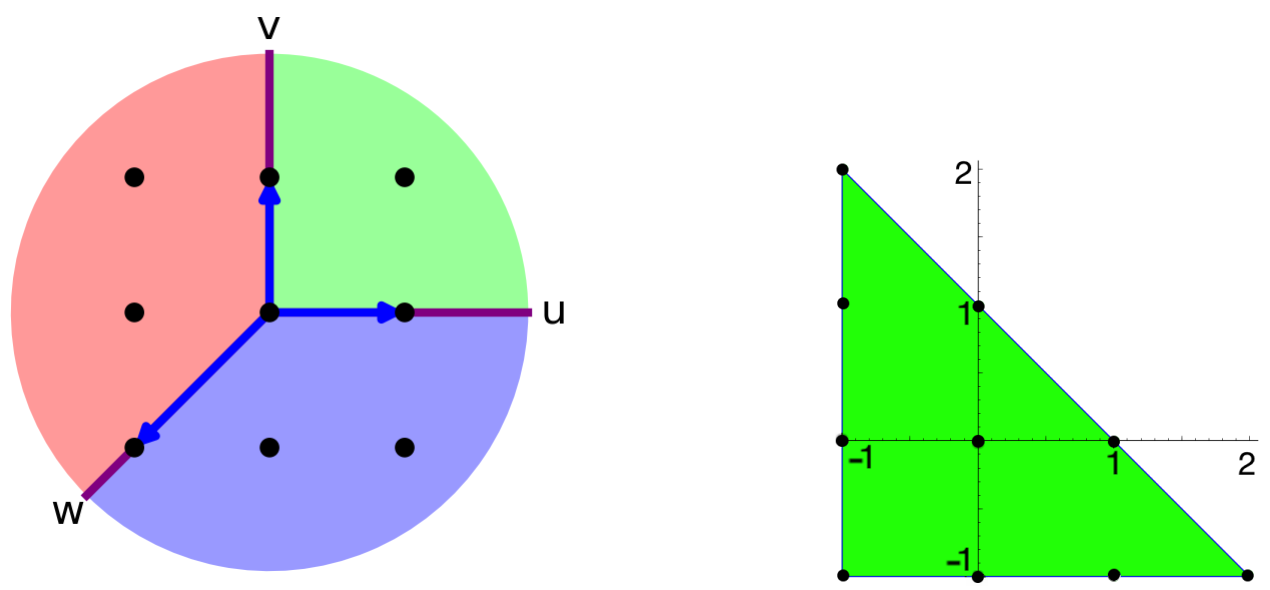

Figure 1: Fan of $\mathbb{P}^{2}$ on the left and its dual polytope on the right.

the coefficients $s_{i}$ that are generically not defined over the field $K$. Thus, we specialize the constraint 3.2 by setting the coefficients $s_{4}$ and $s_{10}$ to zero. This specialization can also be viewed as changing the toric ambient variety such that the coefficients $s_{4}$ and $s_{10}$ are automatically absent by means of the toric construction. This is achieved by going from $\mathbb{P}^{2}$ to $d P_{2}$, which is the blow-up of $\mathbb{P}^{2}$ at two generic points. In figure 2 we have depicted the polytope of $d P_{2}$ for the blow-up at $[u: v: w]=[0: 0: 1]$ and $[u: v: w]=[0: 1: 0]$. We note that the blow-ups introduces new exceptional divisors $E_{i}$ with coordinates $e_{i}, i=1,2$.
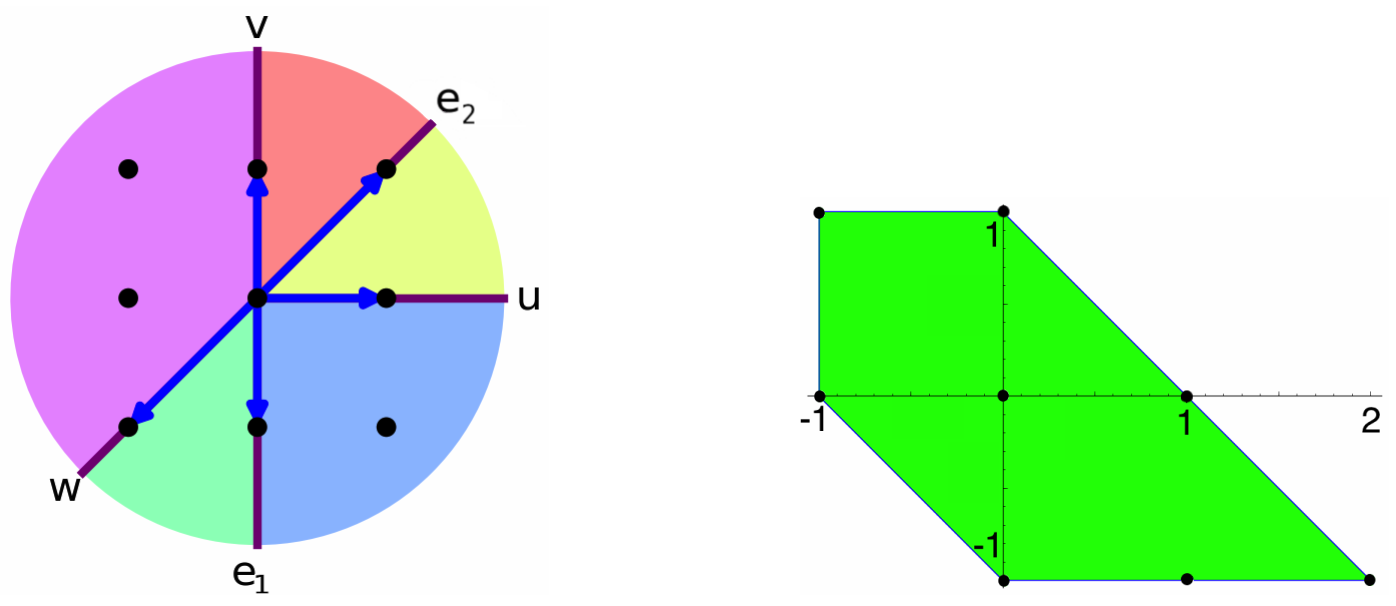

Figure 2: Fan of $d P_{2}$ on the left and its dual polytope on the right. 
This latter perspective on the specialization of the constraint $(3.2)$ as the CalabiYau hypersurface in $d P_{2}$ is of particular relevance for the construction of smooth elliptic fibrations in F-theory. However, the blown-up geometry $d P_{2}$ is of no importance for the determination of the Weierstrass model of the curve elliptic curve $\mathcal{E}$ with the rational points $Q$ and $R$, with which we proceed in the remainder of this section. Thus, we discuss the resolved geometry of $d P_{2}$ with all divisor classes including the exceptional divisors $E_{i}$ separately in section 3.2 and work for the following in the patch $e_{1}=1, e_{2}=1$.

The restricted hypersurface following from (3.2) with $s_{4}=s_{10}=0$ takes now the form

$$
p \equiv s_{1} u^{3}+s_{2} u^{2} v+s_{3} u v^{2}+s_{5} u^{2} w+s_{6} u v w+s_{7} v^{2} w+s_{8} u w^{2}+s_{9} v w^{2}=0 .
$$

Then, this hypersurface constraint specializes at $u=0$ to

$$
v w\left(s_{7} v+s_{9} w\right)=0,
$$

which vanishes at the three different points with coordinates

$$
P=[0: 0: 1], \quad Q=[0: 1: 0], \quad R=\left[0: s_{9}:-s_{7}\right] .
$$

We emphasize that the points $P$ and $Q$ coincide with precisely those points at which we blow up $\mathbb{P}^{2}$ into $d P_{2}$, see section 3.2 for a more detailed discussion.

We note that we could set even more coefficients $s_{i}$ to zero or to one by using the automorphisms of the ambient space, e.g. by shifting or rescaling variables and the equation, if we were interested in the properties of the elliptic curve alone. For example the shift symmetry of $w$ by an appropriate factor of $u$ is still unbroken in the presence of the rational points $Q$ and $R$ since these are left invariant. For example, we can perform the transformation $w \rightarrow w^{\prime}-\frac{s_{5}}{2 s_{8}} u$ to eliminate the term $u^{2} w$ in $(3.4)^{10}$. However, we use later in section 6 the elliptic curve defined by (3.4) as the general elliptic fiber in an elliptically fibered Calabi-Yau manifold. Then the coefficients $s_{i}$ are lifted to sections over the base of the fibration. In this case the embedding of the fiber into the Calabi-Yau manifold will in general forbid eliminating more $s_{i}$ than those in (3.4). This can be seen explicitly in the toric example constructed in section 6 . Therefore, we work with the most general form (3.4) of an elliptic curve with two rational points.

${ }^{10}$ The coefficients will take the form

$$
s_{1}^{\prime}=s_{1}-\frac{s_{5}^{2}}{4 s_{8}}, \quad s_{2}^{\prime}=s_{2}-\frac{s_{5} s_{6}}{2 s_{8}}+\frac{s_{5}^{2} s_{9}}{4 s_{8}^{2}}, \quad s_{3}^{\prime}=s_{3}-\frac{s_{5} s_{7}}{2 s_{8}}, \quad s_{6}^{\prime}=s_{6}-\frac{s_{5} s_{9}}{s_{8}},
$$

with the other coefficients unchanged. 
To obtain the Weierstrass equation with respect to $P$, we need to find the three sections of $H^{0}(k P)=H^{0}(k M-k Q-k R)$ for $k=1,2,3$. These sections are precisely the projective coordinates $z, \tilde{x}, \tilde{y}$ of the Weierstrass model in $\mathbb{P}^{2}(1,2,3)$. For the purpose of constructing these sections we determine sections of $H^{0}(k M)$ on the ambient space $d P_{2}$ that simultaneously vanish $k$ times both at $Q$ and $h^{11}$ when restricted to $\mathcal{E}$.

The general derivation of this birational transformation from the cubic (3.4) to the Tate model (2.1) and then the Weierstrass form (2.2) is quite lengthy. Therefore we first summarize the results and present the proof in the remainder of the section for the interested reader. The birational map from the projective coordinates $[u: v: w]$ of $d P_{2}$ to $[z: \tilde{x}: \tilde{y}]$ in the Weierstrass model $(2.2)$ reads

$$
\begin{aligned}
z= & u \\
\tilde{x}= & s_{3} s_{9} u v+\left(s_{6} s_{9}-s_{7} s_{8}\right) u w+s_{7} s_{9} v w+s_{9}^{2} w^{2}+\left(\frac{1}{12} s_{6}^{2}-\frac{1}{3} s_{3} s_{8}-\frac{1}{3} s_{5} s_{7}+\frac{2}{3} s_{2} s_{9}\right) z^{2} \\
\tilde{y}= & \frac{1}{2}\left[s_{3}\left(s_{6} s_{9}-2 s_{7} s_{8}\right) u^{2} v+\left(s_{6}^{2} s_{9}-s_{6} s_{7} s_{8}+2 s_{9}\left(-s_{5} s_{7}-s_{3} s_{8}+s_{2} s_{9}\right)\right) u^{2} w\right. \\
& +\left(s_{6} s_{7} s_{9}-2 s_{7}^{2} s_{8}+2 s_{3} s_{9}^{2}\right) u v w+\left(3 s_{6} s_{9}^{2}-4 s_{7} s_{8} s_{9}\right) u w^{2}+s_{7} s_{9}^{2} v w^{2}+2 s_{9}^{3} w^{3} \\
& \left.+\left(s_{2} s_{6} s_{9}-s_{2} s_{7} s_{8}-s_{3} s_{5} s_{9}-s_{1} s_{7} s_{9}\right) z^{3}\right] .
\end{aligned}
$$

The sections defined like this obey the Weierstrass constraint (2.2),

$$
\tilde{y}^{2}=\tilde{x}^{3}+f \tilde{x} z^{4}+g z^{6}
$$

with the parameters $f$ and $g$ determined via $(2.4)$ in terms of the three polynomials

$$
\begin{aligned}
b_{2}= & s_{6}^{2}-4 s_{5} s_{7}-4 s_{3} s_{8}+8 s_{2} s_{9}, \\
b_{4}= & 2 s_{2}^{2} s_{9}^{2}+s_{1} s_{7}\left(2 s_{7} s_{8}-s_{6} s_{9}\right)+s_{2}\left(s_{6}^{2} s_{9}-s_{6} s_{7} s_{8}-2 s_{5} s_{7} s_{9}\right) \\
& +s_{3}\left(2 s_{5} s_{7} s_{8}-s_{5} s_{6} s_{9}-2 s_{2} s_{8} s_{9}+2 s_{1} s_{9}^{2}\right), \\
b_{6}= & \left(s_{2} s_{7} s_{8}+s_{3} s_{5} s_{9}-s_{2} s_{6} s_{9}+s_{1} s_{7} s_{9}\right)^{2} \\
& -4 s_{1} s_{3}\left(s_{7}^{2} s_{8}^{2}+s_{9}^{2}\left(s_{3} s_{8}-s_{2} s_{9}\right)+s_{7} s_{9}\left(s_{5} s_{9}-s_{6} s_{8}\right)\right) .
\end{aligned}
$$

\footnotetext{
${ }^{11}$ We note that this somewhat counter-intuitive fact follows from the fact that we implicitly divide by a function $g$ vanishing at $P+Q+R$. Indeed, we recall the definition of the line bundle associated to a Cartier divisor $D, \mathcal{O}(D):=\left\{\frac{f}{g} \mid \operatorname{div}\left(\frac{f}{g}\right)+D \geq 0\right\}$ for local functions $f, g$, cf. [58]. Applying this for $\mathcal{O}(P+Q+R)$ we obtain that $g$ has three zeros precisely at $P, Q, R$, with $f$ of degree three vanishing elsewhere. For $\mathcal{O}(P)$ we obtain $g$ has to vanish at $P$ and $f$ is of degree one. Then, sections of $\mathcal{O}(P)$ are a subset of sections of $\mathcal{O}(P+Q+R)$ by demanding that $f$ in the latter vanishes precisely at $Q$ and $R$.
} 
We have summarized the somewhat lengthy expressions for $f, g$ along with the Tate coefficient $a_{i}$ and the discriminant in the appendix $\mathrm{A}$.

Along similar lines outlined below, we obtain the coordinates for the generators of the Mordell-Weil group of $\mathcal{E}$ given by the rational points $Q$ and $R$ in Weierstrass form. The result reads

$Q=\left[\tilde{x}_{Q}: \tilde{y}_{Q}: z_{Q}\right]=\left[\frac{1}{12}\left(s_{6}^{2}-4 s_{5} s_{7}+8 s_{3} s_{8}-4 s_{2} s_{9}\right), \frac{1}{2}\left(s_{3} s_{6} s_{8}-s_{2} s_{7} s_{8}-s_{3} s_{5} s_{9}+s_{1} s_{7} s_{9}\right): 1\right]$

for $Q$ and analogously for $R=\left[\tilde{x}_{R}: \tilde{y}_{R}: z_{R}\right]$,

$$
\begin{aligned}
\tilde{x}_{R}= & \frac{1}{12}\left(12 s_{7}^{2} s_{8}^{2}+s_{9}^{2}\left(s_{6}^{2}+8 s_{3} s_{8}-4 s_{2} s_{9}\right)+4 s_{7} s_{9}\left(-3 s_{6} s_{8}+2 s_{5} s_{9}\right)\right), \\
\tilde{y}_{R}= & \frac{1}{2}\left(2 s_{7}^{3} s_{8}^{3}+s_{3} s_{9}^{3}\left(-s_{6} s_{8}+s_{5} s_{9}\right)+s_{7}^{2} s_{8} s_{9}\left(-3 s_{6} s_{8}+2 s_{5} s_{9}\right)\right. \\
& +s_{7} s_{9}^{2}\left(s_{6}^{2} s_{8}+2 s_{3} s_{8}^{2}-s_{5} s_{6} s_{9}-s_{2} s_{8} s_{9}+s_{1} s_{9}^{2}\right) \\
z_{R}= & s_{9} .
\end{aligned}
$$

In the remainder of the section we derive the final results $(3.8)$ and $(3.10)$. The reader less interest in these details can safely skip to section 3.2 . Beginning with $H^{0}(M)$, we already know that the section $u=0$, by assumption, vanishes at the three points $P, Q$ and $R$. Thus we set

$$
z:=u
$$

Next we need two find a section of $H^{0}(2 M)$ with double zeros at $Q$ and $R$ on $\mathcal{E}$. There are two such sections, one of which is given by the section $u^{2}=z^{2}$. The other one is constructed from an appropriate linear combination of the other elements of $H^{0}(2 M)$. We make the ansatz

$$
x:=a v^{2}+b u v+c w^{2}+d v w+e u w .
$$

The coefficients are fixed requiring that the section $x$ vanishes at degree two on both points $Q$ and $R$. This gives us four equations. The other coefficient can be rescaled away using the scaling of $x$.

A nice pictorial way to think about these conditions on the coefficients in $x$ is to realize that $x=0$ and $p=0$ in (3.4) are two curves in the ambient space $d P_{2}$. Then the first two of the four conditions on the coefficients enforce that $x$ vanishes at the points $P, Q$, which are also automatically the intersections of $p=0$ and $x=0$. The other two conditions on the coefficients then make $Q, R$ a double zero or equivalently let the curves intersect tangentially. 
A systematic way of finding the coefficients is to solve for one of the variables $u, v$, $w$ in one of the equations $p=0, x=0$ and then to use the other one to determine the unknown coefficients in 3.14 in order to obtain the right order of vanishing. First we note that both $Q$, and $R$ can be described in the affine patch $v=1$. To solve for one of the remaining variables $u, w$ instead of dealing with radicals we can approximate the curve $\mathcal{E}$ by a Taylor expansion. We solve for $w \equiv w(u)$ order by order in $u$ by considering $f(u, 1, w(u))=0$ as an implicit function for $w$ in terms of $u$. Expanding the curve $p=0$ first around $Q$, then around $R$ with coordinates (3.6) we obtain, omitting terms of third order and higher in $u$,

$$
\begin{aligned}
& Q: w=-\frac{s_{3}}{s_{7}} u-\frac{1}{s_{7}}\left(s_{2}-\frac{s_{3} s_{6}}{s_{7}}+\frac{s_{3}^{2} s_{9}}{s_{7}^{2}}\right) u^{2} \\
& R: w=-\frac{s_{7}}{s_{9}}+\left(\frac{s_{3}}{s_{7}}+\frac{s_{7} s_{8}-s_{6} s_{9}}{s_{9}^{2}}\right) u+\left(\frac{s_{2}}{s_{7}}-\frac{s_{3} s_{6}}{s_{7}^{2}}+\frac{s_{3}^{2} s_{9}}{s_{7}^{3}}-\frac{s_{7} s_{8}^{2}-s_{6} s_{8} s_{9}+s_{5} s_{9}^{2}}{s_{9}^{3}}\right) u^{2} .
\end{aligned}
$$

Now we can pull back the section $x$ to the curve $\mathcal{E}$ by simply plugging the solution for $w(u)$ into the ansatz (3.14) for $x$. Since we require an order of vanishing of two we have to set the coefficients of $u^{0}$ and $u^{1}$ to zero. Employing the expansion (3.15) at $Q$ yields

$$
a=0, \quad b-\frac{s_{3}}{s_{7}} d=0 .
$$

Proceeding similarly around $R$ using (3.15), we obtain two further equations

$$
a+\frac{s_{7}^{2}}{s_{9}^{2}} c-\frac{s_{7}}{s_{9}} d=0, \quad b+\frac{s_{7}^{2} s_{8}-s_{6} s_{7} s_{9}+s_{3} s_{9}^{2}}{s_{7} s_{9}^{2}} d+2 \frac{s_{6} s_{7} s_{9}-s_{7}^{2} s_{8}-s_{3} s_{9}^{2}}{s_{9}^{3}} c-\frac{s_{7}}{s_{9}} e=0 .
$$

Solving these equations, setting $c=1$ by rescaling and cleaning denominators in $x$, we finally get the section

$$
x=s_{3} s_{9} u v+\left(s_{6} s_{9}-s_{7} s_{8}\right) u w+s_{7} s_{9} v w+s_{9}^{2} w^{2} .
$$

Next, we need three elements of $H^{0}(3 M)$ with triple zeros at $Q$ and $R$ on the curve $\mathcal{E}$. We know ten sections of this bundle, only nine are independent and we already know two combinations that vanish appropriately, namely $u^{3}$ and $u x$. The other possible section has to be a linear combination of the remaining seven sections and we make the ansatz

$$
y:=a u^{2} v+b v^{3}+c w^{3}+d v^{2} w+e u w^{2}+f v w^{2}+g u v w .
$$


Proceeding in the same way as before, we use the solutions (3.15) to pull the section $y$ to the curve $\mathcal{E}$, but now require that it vanishes at order $u^{3}$ both at $Q$ and $R$. This yields three conditions for each point, i.e. a total of six conditions, by demanding that the terms $u^{0}, u^{1}$ and $u^{2}$ are absent. Setting $c=1$ by rescaling $y$ and cleaning some denominators, we obtain

$$
\begin{aligned}
y= & \frac{s_{3}\left(s_{7}^{2} s_{8}^{2}-s_{6} s_{7} s_{8} s_{9}+s_{5} s_{7} s_{9}^{2}+s_{3} s_{8} s_{9}^{2}-s_{2} s_{9}^{3}\right)}{s_{6} s_{9}-s_{7} s_{8}} u^{2} v \\
& +\frac{s_{7}^{3} s_{8}^{2}-s_{6} s_{7}^{2} s_{8} s_{9}+s_{5} s_{7}^{2} s_{9}^{2}+s_{3} s_{6} s_{9}^{3}-s_{2} s_{7} s_{9}^{3}}{s_{6} s_{9}-s_{7} s_{8}} u v w+s_{7} s_{9}^{2} v w^{2} \\
& +\frac{s_{9}\left(2 s_{7}^{2} s_{8}^{2}-3 s_{6} s_{7} s_{8} s_{9}+s_{6}^{2} s_{9}^{2}+s_{5} s_{7} s_{9}^{2}+s_{3} s_{8} s_{9}^{2}-s_{2} s_{9}^{3}\right)}{s_{6} s_{9}-s_{7} s_{8}} u w^{2}+s_{9}^{3} w^{3} .
\end{aligned}
$$

Now that we know the form of the sections $x, y$ we can determine the Tate model (2.1) by plugging in the solutions (3.18), (3.20) and solving for the Tate coefficients $a_{i}$. We note that in order to find a solution we have to reduce the Tate form, which is a degree six polynomial in $u, v, w$, in the ideal generated by the original cubic constraint (3.4). We present the results for the $a_{i}$ in (A.2) of appendix A from which we immediately calculate the coefficients $b_{2}, b_{4}$ and $b_{6}$ in (3.10). From the Tate model, the Weierstrass form (2.2) can be obtained by the variable transformation (2.3) that takes the form of (3.8) as claimed. We readily obtain the functions $f$ and $g$ in the Weierstrass model and the discriminant and refer to appendix $\mathrm{A}$ for their explicit form.

Next, we focus on finding the rational points $Q$ and $R$ in Weierstrass form. Recalling that there is an implicit multiplication by a meromorphic function with poles of degree $k$ in each case, we obtain the coordinates for $Q(R)$ if we look for a section $x^{\prime}$ of degree two that vanishes three times at $Q(R)$. We have to include all elements of $H^{0}(2 M)$ :

$$
x^{\prime}:=a v^{2}+b u v+c w^{2}+d v w+e u w+\tilde{f} u^{2}, .
$$

The new parameter $\tilde{f}$ is fixed by the additional constraint from the vanishing of the coefficient of $u^{2}$, while all the other coefficients take the same values as before. Noting that $z \equiv u$, we then obtain

$$
x^{\prime}=x-\left(s_{3} s_{8}-s_{2} s_{9}\right) z^{2} \stackrel{!}{=} 0
$$

at $Q$ which fixes the $x$-coordinate of $Q$. After scaling and going to the Weierstrass coordinates we obtain the generator as in (3.11), where the $\tilde{y}$-coordinate of $Q$ is obtained by solving $(3.9)$ for $\tilde{y}$ given $\tilde{x}_{Q}$. Following the same procedure for the point $R$ we confirm the result (3.12). 


\subsection{Resolved Elliptic Curve in $d P_{2}$ and its Elliptic Fibrations}

In this section we discuss the key geometric properties of the resolution of the elliptic curve (3.4) in $d P_{2}$. To this end we have to take into account the exceptional divisors $E_{i}$ and their sections $e_{i}, i=1,2$ that we have set to one in the previous discussion. Then, smoothness of $\mathcal{E}$ is ensured by the toric construction since the curve (3.4) lifts to the generic CalabiYau onefold in $d P_{2}$. The smoothness property even holds in elliptic fibrations $X$ with general fiber given by $d P_{2}$, if the most generic toric Calabi-Yau hypersurface is considered. Indeed, as we discuss explicitly in section 4 the divisors $E_{1}, E_{2}$ resolve the singularities of the fibration at codimension two and higher. In particular, this allows us to determine the matter spectrum in F-theory from the splitting of the elliptic curve in $d P_{2}$ over codimension two from the presence of the new projective coordinates $e_{i}$.

We construct $d P_{2}$ as follows. We blow up $\mathbb{P}^{2}$ at the two points $[u: v: w]=[0: 1: 0]$ and $[u: v: w]=[0: 0: 1]$ by introducing two new projective coordinates $e_{1}, e_{2}$ as

$$
u=u^{\prime} e_{1} e_{2}, \quad v=v^{\prime} e_{2}, \quad w=w^{\prime} e_{1} .
$$

This is the blow-down map $\pi: d P_{2} \rightarrow \mathbb{P}^{2}$, where $e_{1}, e_{2}$ are holomorphic sections vanishing at the exceptional divisors $E_{1}, E_{2}$. We see that the original codimension two loci $u=$ $w=0$, respectively, $u=v=0$ in $\mathbb{P}^{2}$ are replaced by $e_{1}=0$, respectively, $e_{2}=0$, i.e. by entire divisors $E_{i}$ with the geometry of $\mathbb{P}^{1}$. We summarize the toric realization of $d P_{2}$ encoded in its polytope $\Delta_{d P_{2}}$ and its homogeneous coordinates as

\begin{tabular}{|c|rr||c|c|}
\hline & vertices & & divisor class \\
\hline$\nu_{1}$ & 1 & 0 & $u^{\prime}$ & $H-E_{1}-E_{2}$ \\
$\nu_{2}$ & 0 & 1 & $v^{\prime}$ & $H-E_{2}$ \\
$\nu_{3}$ & -1 & -1 & $w^{\prime}$ & $H-E_{1}$ \\
$\nu_{4}$ & 0 & -1 & $e_{1}$ & $E_{1}$ \\
$\nu_{5}$ & 1 & 1 & $e_{2}$ & $E_{2}$ \\
\hline
\end{tabular}

Here we denote the vertices of $\Delta_{d P_{2}}$ by $\nu_{i}$ in the first row and write them explicitly as two-dimensional row-vectors in the second and third column as depicted in the polytope in figure 2. The only integral internal point is the origin. In the third and fourth column we summarize the homogeneous coordinates on $d P_{2}$ and their divisor classes, where $H$ is the pullback of the hyperplane class on $\mathbb{P}^{2}$ and the $E_{i}$ are the exceptional divisors. This in particular implies that the anti-canonical bundle $K_{d P_{2}}^{-1}$ of $d P_{2}$, which is computed as the negative of the sum of all toric divisors, has changed to $K_{d P_{2}}^{-1}=\mathcal{O}\left(3 H-E_{1}-E_{2}\right)$, compared to $K_{\mathbb{P}^{2}}^{-1}=\mathcal{O}(3 H)$ before the blow-ups. 
We note the intersections in the star triangulation of $\Delta_{d P_{2}}$ with star given by the origin as

$$
H^{2}=1, \quad H \cdot E_{i}=0, \quad E_{i} \cdot E_{j}=-\delta_{i j} .
$$

Here we made use of the exceptional set, the Stanley-Reissner ideal $S R$ of all vertices not sharing a two dimensional cone, reading

$$
S R=\left\{u^{\prime} v^{\prime}, u^{\prime} w^{\prime}, e_{1} e_{2}, e_{1} v^{\prime}, e_{2} w^{\prime}\right\}
$$

We observe that the polytope of $\mathbb{P}^{2}$ is embedded by the first three points $\nu_{1}, \nu_{2}$ and $\nu_{3}$. The addition of the vertices $\nu_{4}, \nu_{5}$ not only introduces the divisors $E_{i}$ but also removes the corners $(-1,2)$ and $(-1,-1)$ of the dual polytope of $\mathbb{P}^{2}$, compare figures 1 and 2 . These corners correspond precisely to the monomials $s_{4} v^{3}$ and $s_{10} w^{3}$ in $\mathbb{P}^{2}$, that are no longer admissible sections of the anti-canonical bundle on $d P_{2}$. As one can see by either looking at dual polytope 2 or by writing down all sections of the anti-canonical bundle $\mathcal{O}\left(3 H-E_{1}-E_{2}\right)$ of $d P_{2}$ by hand with the charge assignments in (3.24), the generic Calabi-Yau hypersurface $p^{\prime}$ on $d P_{2}$ reads

$p^{\prime}=u^{\prime}\left(s_{1} u^{\prime 2} e_{1}^{2} e_{2}^{2}+s_{2} u^{\prime} v^{\prime} e_{1} e_{2}^{2}+s_{3} v^{\prime 2} e_{2}^{2}+s_{5} u^{\prime} w^{\prime} e_{1}^{2} e_{2}+s_{6} v^{\prime} w^{\prime} e_{1} e_{2}+s_{8} w^{\prime 2} e_{1}^{2}\right)+s_{7} v^{\prime 2} w^{\prime} e_{2}+s_{9} v^{\prime} w^{\prime 2} e_{1}$.

Now we investigate the pullback under $\pi$ of the points $P, Q$ and $R$ in (3.6) that we by abuse of notation denote by the same symbols. We readily infer from the map 3.23 that the section $u \in \mathcal{O}(P+Q+R)$ splits into three components as expected and we identify $P$ as $e_{2}=0, Q$ as $e_{1}=0$ and the image of $R$ as $D_{u^{\prime}}:=\left\{u^{\prime}=0\right\}$. The value of all coordinates in the notation $\left[u^{\prime}: v^{\prime}: w^{\prime}: e_{1}: e_{2}\right]$ is obtained by inserting these components into the hypersurface equation (3.27) and setting all coordinates to 1 that do not intersect the component under consideration due to the exceptional set (3.26). This yields

$$
\begin{gathered}
P: E_{2} \cap p^{\prime}=\left[-s_{9}: s_{8}: 1: 1: 0\right], \quad Q: E_{1} \cap p^{\prime}=\left[-s_{7}: 1: s_{3}: 0: 1\right] \\
R: D_{u^{\prime}} \cap p^{\prime}=\left[0: 1: 1:-s_{7}: s_{9}\right]
\end{gathered}
$$

for the coordinates of the points $P, Q$ and $R$ on the resolved curve in $d P_{2}$.

We emphasize the dependence of the points $P, Q, R$ on the $s_{i}$, in particular the situation when certain $s_{i}$ vanish. Note that it is manifest in the form (3.28), that the points $P, Q$ and $R$ are ill-defined if $s_{i}=s_{j}=0$ simultaneously for the combinations $(i, j)$ in (3.28) due to the Stanley-Reissner ideal (3.26). In particular, when considering 
elliptic fibrations $\hat{X}$ with elliptic fiber given by 3.27$)$ this translates to a special behavior of the corresponding sections $\hat{s}_{P}, \hat{s}_{Q}, \hat{s}_{R}$ at codimension two loci $s_{i}=s_{j}=0$. Note that the $s_{i}$ lift to sections on the base $B$ of the fibration $\hat{X}$, see the next paragraph, and can generically vanish. As we discuss in detail in the next section 4 this behavior is the key to understand the matter spectrum of an F-theory compactification on $\hat{X}$.

We would like to conclude by discussing the general construction of an elliptic fibration $\hat{X}$ over a base $B$. For concreteness we focus on fibrations over $B=\mathbb{P}^{2}$ noting that the following can readily be generalized to different bases. First, we construct all fibrations of $d P_{2}$ over $\mathbb{P}^{2}$. The total space of this fibration of toric varieties is classified only by two integers, that we denote by $n_{2}$ and $n_{12}$ for reasons that will become clear below. We denote the total space as

$$
d P_{2} \rightarrow d P_{2}\left(n_{2}, n_{12}\right) \rightarrow \mathbb{P}^{2} .
$$

It is clear that the fibration $d P_{2}\left(n_{2}, n_{12}\right)$ is only specified by two integers because we can associate each projective coordinates $u^{\prime}, v^{\prime}, w^{\prime}, e_{1}, e_{2}$ to a different line bundle $\mathcal{O}\left(k_{i} H_{B}\right)$, $i=1, \ldots, 5$ on $\mathbb{P}^{2}$, where $H_{B}$ is the hyperplane of the $\mathbb{P}^{2}$-base. However, by means of the three $\mathbb{C}^{*}$-actions on $d P_{2}$ we can always eliminate three $k_{i}$ and denote remaining two degrees as $n_{2}, n_{12}$. Then, a possible assignment is

$$
u^{\prime} \in \mathcal{O}\left(\left(n_{2}-3\right) H_{B}\right), \quad v^{\prime} \in \mathcal{O}\left(\left(n_{2}-n_{12}\right) H_{B}\right)
$$

with all other coordinates taking values in the trivial bundle on $\mathbb{P}^{2}$. Next, we note that the anti-canonical bundle of $d P_{2}\left(n_{2}, n_{12}\right)$ is calculated by adjunction as

$$
K_{d P_{2}\left(n_{2}, n_{12}\right)}^{-1}=\mathcal{O}\left(3 H-E_{1}-E_{2}+\left(2 n_{2}-n_{12}\right) H_{B}\right) .
$$

From this and the Calabi-Yau constraint (3.27) we can finally read off the degrees of the coefficients $s_{i}$, that now lift to sections of $\mathbb{P}^{2}$ as well:

\begin{tabular}{|c|c|c|c|c|c|c|c|}
\hline$s_{1}$ & $s_{2}$ & $s_{3}$ & $s_{5}$ & $s_{6}$ & $s_{7}$ & $s_{8}$ & $s_{9}$ \\
\hline $9-n_{2}-n_{12}$ & $6-n_{2}$ & $3+n_{12}-n_{2}$ & $6-n_{12}$ & 3 & $n_{12}$ & $3+n_{2}-n_{12}$ & $n_{2}$ \\
\hline
\end{tabular}

Here the second line denotes the first Chern class or in other words the degree of the section $s_{i}$ with respect to the hyperplane class $H_{B}$ on the base.

Finally, 3.32 illuminates the meaning of the integers $n_{2}$ and $n_{12}$. The sections $s_{7}$ and $s_{9}$ vanish at curves of degree $n_{2}$ respectively $n_{12}$ in $\mathbb{P}^{2}$. But as we see from (3.28) these are precisely the loci where the points $Q$ and $R$, now lifted to rational sections $\hat{s}_{Q}$, 
$\hat{s}_{R}$, respectively the points $P$, also lifted to a section $\hat{s}_{P}$, and $R$ coincide. In other words, these integers calculate the following intersections, cf. (2.17),

$$
n_{2}=\pi\left(S_{P} \cdot S_{R}\right)=S_{P} \cdot S_{R} \cdot H_{B}, \quad n_{12}=\pi\left(S_{Q} \cdot S_{R}\right)=S_{Q} \cdot S_{R} \cdot H_{B},
$$

where we denoted the divisor classes of the sections by capital letters following the conventions of section 2.2 .

\section{Matter Spectrum: Codimension Two Singularities}

In this section we determine the generic matter spectrum of a six-dimensional F-theory compactification on the elliptic threefold $\hat{X}$ over $B$ with the general elliptic fiber $\mathcal{E}$ in $d P_{2}$ found in section 3.1 . We mostly consider the case $B=\mathbb{P}^{2}$, although our discussion is readily generalizable to other geometries.

Determining the matter spectrum requires an analysis of the codimension two singularities of this three-section elliptic fibration ${ }^{12}$. We see that the singularity structure that is generic to these fibrations is completely governed by the behavior of the three sections. This study of the behavior of the sections can be conveniently performed in the Weierstrass model $(3.9)$ of the elliptic curve $\mathcal{E}$. We determine the charges in three steps in subsections $4.1,4.2$ and 4.3 . The multiplicities of matter fields are found in 4.4 . This analysis requires a careful counting of the codimension two points since the loci in the base $B$ supporting matter fields of different charges intersect. The right counting requires subtraction of multiplicities of intersecting loci according to their mutual order of vanishing, which is determined by the resultant of the polynomial systems determining the intersecting codimension two loci. Since the matter is a codimension two effect, the matter charges we find immediately carry over to four-dimensional F-theory compactifications on Calabi-Yau fourfolds. Our analysis extends the methods of [43] to elliptic fibrations with two-section models.

The following discussion is organized by the three qualitatively different codimension two singularities that can occur. The codimension two loci of the first two types are readily found in the Weierstrass model, whereas the third type has to be addressed in the birationally related $d P_{2}$-model of section 3.2 . The three types we distinguish correspond to the following behavior of the sections:

\footnotetext{
${ }^{12}$ We thank Antonella Grassi for helpful discussions on the general study of codimension two singularities of elliptic fibrations and related properties of the discriminant.
} 
- Away from the collision of a section $\hat{s}$ with the zero section $z=0$ in the Weierstrass form, the existence of the section implies the factorization (2.5) of the Weierstrass form. This factorization makes manifest the presence of conifold singularities in $X$. Their resolution leads to matter of charge 1 under the $\mathrm{U}(1)$ associated to $\sigma(\hat{s})$. We discuss the factorized Weierstrass form for the two sections $\hat{s}_{Q}, \hat{s}_{R}$ in section 4.1 .

- Two rational sections $\hat{s}, \hat{s}^{\prime}$ both away from the zero section $z=0$ can collide and contribute charge $(1,1)$-matter under the two associated $\mathrm{U}(1) \times \mathrm{U}(1)$-symmetry. This behavior is discussed in section 4.2 .

- A section $\hat{s}$ can be ill-defined at codimension two. This is easily seen in the $d P_{2^{-}}$ model of section 3.2 . The section $\hat{s}$ is ill-defined if its corresponding point $(3.28)$ in the fiber passes through the Stanley-Reissner ideal (3.26) of $d P_{2}$. This happens at codimension two loci $s_{i}=s_{j}=0$ and we have to blow-up the base $B$ at these loci. Our discussion in section 4.3 is organized according to which of these combinations vanish. Then the section wraps a $\mathbb{P}^{1}$ fiber component, which is precisely the toric divisor in $d P_{2}$ corresponding to the ray that subdivides the $2 \mathrm{~d}$-cone corresponding to the two coordinates in the Stanley-Reissner ideal (3.26) .

All these singularities have in common that their resolved fiber is of $I_{2}$-type, i.e. an $\mathrm{SU}(2)$-fiber with the original singular curve and one isolated $\mathbb{P}^{1}$. The matter hypermultiplets in six dimensions arise from the isolated rational curve $\mathbb{P}^{1}$ at the resolved codimension two singularities of the Calabi-Yau manifold. The intersection structure of the resolved fibers with the sections $\hat{s}_{P}, \hat{s}_{Q}, \hat{s}_{R}$ determines the pattern of U(1)-charges of the matter. The multiplicities of matter fields is computed by counting the number of solutions to the codimension two constraints as demonstrated in section 4.4. We summarize our findings in table 4.1 .

We begin our analysis by summarizing the basics of the construction of a (singular) elliptic fibration $X$ over $B=\mathbb{P}^{2}$ in Weierstrass form (2.2). We follow the general discussion of section 2.2. The Weierstrass form of $X$ is considered as the Calabi-Yau hypersurface in the total space of projective bundle $\mathbb{P}^{2}\left(\mathcal{O}_{B} \oplus \mathcal{L}^{2} \oplus \mathcal{L}^{3}\right)$ with coordinates $[z: x: y]^{13}$, By the Calabi-Yau condition we identify $\mathcal{L}=K_{B}^{-1}=\mathcal{O}_{\mathbb{P}^{2}}(3)$ as the anti-canonical bundle and $\mathcal{O}_{B}=\mathcal{O}_{\mathbb{P}^{2}}(0)$ as the trivial line bundle on $\mathbb{P}^{2}$. The parameters $f$ and $g$ in 2.2 lift to sections of $\mathcal{O}_{\mathbb{P}^{2}}(12)$ and $\mathcal{O}_{\mathbb{P}^{2}}(18)$. The zero section is located at $[z: x: y]=[0: 1: 1]$.

\footnotetext{
${ }^{13}$ In contrast to section 2 we denote here the coordinates in Weierstrass form by $(x, y) \equiv(\tilde{x}, \tilde{y})$.
} 


\begin{tabular}{|c||c|}
\hline$\left(q_{1}, q_{2}\right)$ & Multiplicity \\
\hline \hline$(1,0)$ & $54-15 n_{2}+n_{2}^{2}+\left(12+n_{2}\right) n_{12}-2 n_{12}^{2}$ \\
\hline$(0,1)$ & $54+2\left(6 n_{2}-n_{2}^{2}+6 n_{12}-n_{12}^{2}\right)$ \\
\hline$(1,1)$ & $54+12 n_{2}-2 n_{2}^{2}+\left(n_{2}-15\right) n_{12}+n_{12}^{2}$ \\
\hline$(-1,1)$ & $n_{12}\left(3-n_{2}+n_{12}\right)$ \\
\hline$(0,2)$ & $n_{2} n_{12}$ \\
\hline$(-1,-2)$ & $n_{2}\left(3+n_{2}-n_{12}\right)$ \\
\hline
\end{tabular}

Table 4.1: Matter spectrum with $\mathrm{U}(1) \times \mathrm{U}(1)$-charges $\left(q_{1}, q_{2}\right)$ in the first and multiplicities in the second column. The integers $n_{2}, n_{12}$ are defined in (3.33).

The two Mordell-Weil generators (3.11) and (3.12) are of the form

$$
\hat{s}: \quad[x, y, z]=\left[g_{2 n+6}, g_{3 n+9}, g_{n}\right],
$$

where the subscript indicates the degree of the polynomials on $\mathbb{P}^{2}$. The intersection of the section $\hat{s}$ with the zero section $z=0$ in the Weierstrass model is the integer $n$, and we denote it by $n_{1}, n_{2}$ for the sections $\hat{s}_{Q}$ and $\hat{s}_{R}$ respectively ${ }^{14}$. The intersection of $\hat{s}_{Q}$, $\hat{s}_{R}$ is denoted as $n_{12}$, cf. (3.33).

\subsection{Factorized Weierstrass Form: charges $(1,0)$ and $(0,1)$}

Charge one loci occur when the singular point of the degenerated fiber coincides with the point marked by a section $\hat{s}$, see figure 3 . This ensures, that on the resolution the exceptional $\mathbb{P}^{1}$ in the fiber intersects the section $\hat{s}$ and render the hypermultiplet charged under the $\mathrm{U}(1)$ corresponding to $\hat{s}$. In our case, each of the sections $\hat{s}_{Q}, \hat{s}_{R}$ can pass separately through the singular point and its resolving $\mathbb{P}^{1}$, thus, giving rise to matter of charge $(1,0)$ respectively $(0,1)$ under $\mathrm{U}(1) \times \mathrm{U}(1)$. See section 4.2 for the case when both sections go through the singular point simultaneously.

We begin by spelling out the general strategy for the identification of these loci and specialize to $\hat{s}_{Q}, \hat{s}_{R}$ below. We emphasize that the structure of the Weierstrass model of the fibration $X$ with fiber $\mathcal{E}$ with two sections implies a particular factorization. This facilitates the search for codimension two singularities in the fiber in $X$ that coincide

\footnotetext{
${ }^{14} n_{1}=0$ in our class of models.
} 

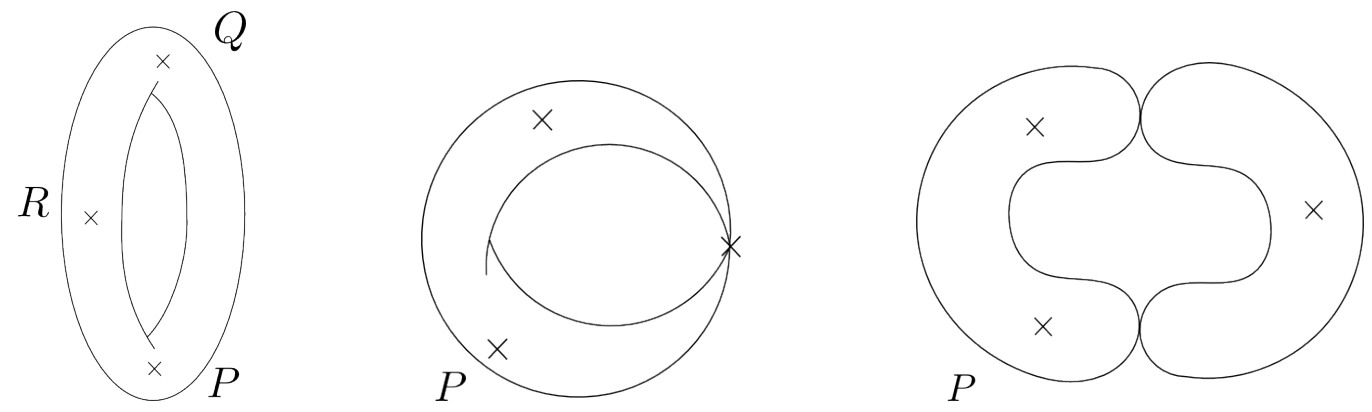

Figure 3: On the left it is shown a regular fiber with rational sections at generic points. In the center, a singular fiber is displayed with a section crossing the singularity where charged matter sits. On the right, the curve is shown after resolution. The isolated curve gives rise to an hypermultiplet charged under the corresponding Abelian gauge field.

with the point marked by the section $\hat{s}$. As we have seen in section 2.1 the presence of a rational point on $\mathcal{E}$ with $z=1$ implies the factorization (2.5). Noting the form 4.1) of a section in Weierstrass form, we expect this factorization where $g_{n} \neq 0$. Indeed, the $\mathbb{C}^{*}$-action on $[x: y: z]$ then allows us to cast the Mordell-Weil (MW) generator (4.1) as

$$
\hat{s}:[x, y, z]=\left[g_{6}, g_{9}, 1\right],
$$

where we set $g_{6}=\frac{g_{6+2 n}}{g_{n}^{2}}, g_{9}=\frac{g_{9+3 n}}{g_{n}^{3}}$. Then, the Weierstrass model factors in the form

$$
p_{1}:=-\left(y^{2}-g_{9}^{2}\right)+\left(x-g_{6}\right)\left(x^{2}+g_{6} x+g_{12}\right)=0,
$$

with appropriate $g_{12}$, cf. (2.6).

This is a singular Weierstrass model with singularities located at the points satisfying $p_{1}=d p_{1}=0$, where $d$ denotes the exterior derivative. The solutions to these equations are

$$
y=0, \quad x=g_{6}, \quad g_{9}=0, \quad \hat{g}_{12}=g_{12}+2 g_{6}^{2}=0 .
$$

We readily confirm that the discriminant (2.6) vanishes quadratically at $g_{9}=\hat{g}_{12}=0$. The singularities are conifold singularities ${ }^{15}$ that are resolved by the birational transformation (3.8). The original singularity at $y=\left(x-g_{6}\right)=0$ is replaced by a rational curve $\mathbb{P}^{1}$ and the whole fiber factors into two rational curves intersecting at two points, i.e. an $I_{2}$-curve. The original singular fiber is identified as the rational curve intersected by the zero section $\hat{s}_{P}$. Then the other curve has to be the isolated exceptional $\mathbb{P}^{1}$. We

\footnotetext{
${ }^{15}$ In the case that $g_{n}=1$ these are the 108 points in the $\mathcal{T}_{0}$ theory found in 43 .
} 
can confirm this identification by using the birational transformation to the Weierstrass form (4.3) and by checking explicitly which curve is mapped to the singular point (4.4) at $y=\left(x-g_{6}\right)=0$. Let us study this explicitly for the two sections $\hat{s}_{Q}, \hat{s}_{R}$ of $X$.

\section{Factorization at the $\hat{s}_{Q}$ section}

The MW-generator of this section has been worked out in equation (3.11). In this case, the section is located at $z=1$ and therefore $z \neq 0$ globally. The Weierstrass equation thus factorizes with

$$
\begin{aligned}
g_{12} & =\frac{1}{72}\left[-s_{6}^{4}+4 s_{6}^{2}\left(2 s_{5} s_{7}+5 s_{3} s_{8}+2 s_{2} s_{9}\right)-36 s_{6}\left(s_{2} s_{7} s_{8}+s_{3} s_{5} s_{9}+s_{1} s_{7} s_{9}\right)\right. \\
& \left.-8\left(2 s_{5}^{2} s_{7}^{2}-s_{3}^{2} s_{8}^{2}+s_{2} s_{3} s_{8} s_{9}+2 s_{2}^{2} s_{9}^{2}+s_{5} s_{7}\left(s_{3} s_{8}-5 s_{2} s_{9}\right)-9 s_{1}\left(s_{7}^{2} s_{8}+s_{3} s_{9}^{2}\right)\right)\right]
\end{aligned}
$$

and the singular loci (4.4) at codimension two are given by

$$
\begin{aligned}
g_{9}^{Q} & =\frac{1}{2}\left(s_{3} s_{6} s_{8}-s_{2} s_{7} s_{8}-s_{3} s_{5} s_{9}+s_{1} s_{7} s_{9}\right) \stackrel{!}{=} 0 \\
\hat{g}_{12}^{Q} & =\frac{1}{2}\left(2 s_{3}^{2} s_{8}^{2}+s_{7}\left(-s_{2} s_{6} s_{8}+2 s_{1} s_{7} s_{8}+2 s_{2} s_{5} s_{9}-s_{1} s_{6} s_{9}\right)\right. \\
& +s_{3}\left(s_{6}^{2} s_{8}-2 s_{5} s_{7} s_{8}-s_{5} s_{6} s_{9}-2 s_{2} s_{8} s_{9}+2 s_{1} s_{9}^{2}\right) \stackrel{!}{=} 0
\end{aligned}
$$

We can use these two constraints to solve for any two coefficients $s_{i}, s_{j}$, as long as the solutions are rational. Plugging these solutions into the resolved elliptic fiber (3.4) reveals its $I_{2}$ nature. In fact, solving and replacing $s_{1}$ and $s_{2}$ yields a split of the fiber class as $\mathcal{E}=c_{1}+c_{2}$ for two rational curves $c_{1}, c_{2}$. The equations of the rational curves read

$$
\begin{array}{ll}
c_{1}: & s_{3} u+s_{7} w=0, \\
c_{2}: & s_{5} s_{7} u^{2}-s_{3} s_{8} u^{2}+s_{6} s_{7} u v-s_{3} s_{9} u v+s_{7}^{2} v^{2}+s_{7} s_{8} u w+s_{7} s_{9} v w=0,
\end{array}
$$

from which it is clear that the curve $c_{2}$ intersects the zero section, $\hat{s}_{P}=[u=0: v=0$ : $w=1]$ and the rational section $\hat{s}_{R}=\left[u=0: v=s_{9}: w=-s_{7}\right]$. Thus $c_{2}$ is identified with the original singular fiber, which is also confirmed by the fact that it maps to the original singular curve (4.3) by the birational map (3.8). The curve $c_{1}$ does not intersect $\hat{s}_{P}$ nor $\hat{s}_{R}$ and is the isolated rational curve, see figure 3 for a depiction of this situation. This is also confirmed by noting that $c_{1}$ is blown-down to the singular point (4.4) by the map (3.8). It thus gives rise to a massless hypermultiplet. Since $c_{1}$ clearly intersects 
the rational section $\hat{s}_{Q}=[u=0: v=1: w=0]$ once, the hypermultiplet, according to 2.20 in the absence of Cartan divisors $D_{i_{I}}$, has $\mathrm{U}(1) \times \mathrm{U}(1)$-charges $\left(q_{1}, q_{2}\right)=(1,0)$ as claimed. We note that we could have performed the same analysis on the resolved geometry (3.27) with the same result.

\section{Factorization at the $\hat{s}_{R}$ section}

The coordinates of this section in Weierstrass form are given in (3.12). We have to take into account that the $z$-coordinate of the section reads $z=s_{9}$ and can vanish. When it is non-zero, the Weierstrass equation factorizes as 4.3 along the loci

$$
\begin{aligned}
g_{9+3 n_{2}}^{R} & =\frac{1}{2}\left[2 s_{7}^{3} s_{8}^{3}+s_{3} s_{9}^{3}\left(-s_{6} s_{8}+s_{5} s_{9}\right)+s_{7}^{2} s_{8} s_{9}\left(-3 s_{6} s_{8}+2 s_{5} s_{9}\right)\right. \\
& \left.+s_{7} s_{9}^{2}\left(s_{6}^{2} s_{8}+2 s_{3} s_{8}^{2}-s_{5} s_{6} s_{9}-s_{2} s_{8} s_{9}+s_{1} s_{9}^{2}\right)\right] \stackrel{!}{=} 0 \\
\hat{g}_{12+4 n_{2}}^{R} & =\frac{1}{2}\left[6 s_{7}^{4} s_{8}^{4}+4 s_{7}^{3} s_{8}^{2} s_{9}\left(2 s_{5} s_{9}-3 s_{6} s_{8}\right)+s_{3} s_{9}^{4}\left(s_{6}^{2} s_{8}+2 s_{3} s_{8}^{2}-s_{5} s_{6} s_{9}-2 s_{2} s_{8} s_{9}+2 s_{1} s_{9}^{2}\right)\right. \\
& -s_{7} s_{9}^{3}\left(s_{6}^{3} s_{8}-s_{5} s_{6}^{2} s_{9}+2 s_{5} s_{9}\left(-3 s_{3} s_{8}+s_{2} s_{9}\right)+s_{6}\left(8 s_{3} s_{8}^{2}+s_{9}\left(-3 s_{2} s_{8}+s_{1} s_{9}\right)\right)\right) \\
& \left.+s_{7}^{2} s_{9}^{2}\left(7 s_{6}^{2} s_{8}^{2}-8 s_{5} s_{6} s_{8} s_{9}+2\left(4 s_{3} s_{8}^{3}+s_{9}\left(-2 s_{2} s_{8}^{2}+s_{5}^{2} s_{9}+s_{1} s_{8} s_{9}\right)\right)\right)\right] \stackrel{!}{=} 0
\end{aligned}
$$

We find the rational solutions of these equations for $s_{1}$ and $s_{2}$. By plugging this into (3.4), we see that the fiber again splits into $\mathcal{E}=c_{1}+c_{2}$ with

$$
\begin{array}{ll}
c_{1}: & -s_{3} s_{9}^{2} u+s_{7}^{2}\left(-s_{8} u+s_{9} v\right)+s_{7} s_{9}\left(s_{6} u+s_{9} w\right)=0, \\
c_{2}: & s_{7}^{2} s_{8}^{2} u^{2}+s_{3} s_{9}^{2} u\left(s_{8} u+s_{9} v\right)+s_{7} s_{9}\left(-s_{6} s_{8} u^{2}+s_{9}\left(s_{5} u^{2}+s_{8} u w+s_{9} v w\right)\right)=0 .
\end{array}
$$

The curve $c_{2}$ intersects the zero section, $\hat{s}_{P}$ and also $\hat{s}_{Q}$, but not $\hat{s}_{R}$. It is the original singular fiber as can also be checked using the birational map (3.8). The curve $c_{1}$ intersects the rational section $\hat{s}_{R}$ at one point but does not intersect $\hat{s}_{P}$ and $\hat{s}_{Q}$. It is blown-down to the singular point 4.4 in the birational map (3.8). Thus it is an isolated rational curve and contributes a hypermultiplet of charges $\left(q_{1}, q_{2}\right)=(0,1)$ according to 2.20 .

\subsection{Doubly-Factorized Weierstrass Form: charge $(1,1)$}

Hypermultiplets charged under both sections can and should exist, both from a geometric point of view and for anomaly cancellation, see section 5. Their location in the base can 
be found by determining singular points of the elliptic fiber $\mathcal{E}$ due to a factorization 4.3 of the Weierstrass form, where in addition both sections $\hat{s}_{Q}, \hat{s}_{R}$ coincide. As we will demonstrate next, this indeed is a codimension two phenomenon.

From the perspective of the $d P_{2}$ fiber, the collision of the two sections happens when $s_{7}=0$. In the Weierstrass form there are even more loci, where the two sections collide and at which the Weierstrass model becomes automatically singular. We first summarize the structure that we find at these loci, before going into specifics. In the appropriate patch, $z=s_{9}$, we first require the coincidence condition $\hat{s}_{Q}=\hat{s}_{R}$, that reads

$$
s_{9}^{2} g_{6}^{Q}-g_{6+2 n_{2}}^{R}=0 .
$$

If this is true, then also $s_{9}^{3} g_{9}^{Q}=g_{9+3 n_{2}}^{R}$ because the $y$-coordinates of the sections $\hat{s}_{Q}, \hat{s}_{R}$ have been determined in section 3.1 by plugging in the $x$ - and $z$-coordinates into the Weierstrass form and solving for $y$. But since $s_{9}^{2} x_{Q}:=s_{9}^{2} g_{6}^{Q}=g_{6+2 n_{2}}^{R}=: x_{R}$, the equality $s_{9}^{3} g_{9}^{Q}=g_{9+3 n_{2}}^{R}$ is automatic. For the loci under consideration, it requires only a single further constraint to make the Weierstrass model singular, which reads

$$
g_{9}:=s_{9}^{3} g_{9}^{Q}=g_{9+3 n_{2}}^{R} \stackrel{!}{=} 0 .
$$

These two conditions (4.14) and (4.15) bring the Weierstrass equation (4.3), using the notation $g_{6} \equiv s_{9}^{2} g_{6}^{Q}=g_{6+2 n_{2}}^{R}$, into the form

$$
y^{2}=\left(x-g_{6}\right)^{2}\left(x-f_{6}\right),
$$

for $f_{6}=-2 g_{6}$, where the double zero at $x=g_{6}, y=0$ is manifest. We note that the polynomial $\hat{g}_{12+4 n_{2}}^{Q}=s_{9}^{4} \hat{g}_{12}^{R}=0$ in (4.6) respectively (4.10) and we see that fiber is automatically singular if the sections coincide and $g_{9}=0$. Here we denote the third root in $x$ by $f_{6}$, however the fiber is smooth $x=f_{6}$.

In our concrete situation, the difference between the polynomials can be read off from (3.11) and (3.12) and takes the form

$$
\delta g_{6}:=s_{9}^{2} g_{6}^{Q}-g_{6+2 n_{2}}^{R}=0=s_{7}\left(s_{7} s_{8}^{2}+s_{9}\left(-s_{6} s_{8}+s_{5} s_{9}\right)\right) .
$$

We see that $s_{7}=0$ is a solution as expected, but also the vanishing of the polynomial in parenthesis makes the sections collide. In the first case, $s_{7}=0$, the fiber becomes singular iff in addition either $s_{3}=0$ or $s_{9}=0$. However, these cases have to be treated differently, as discussed in section 4.3 , because the rational sections are ill-defined at 
these loci. Thus, we assume $s_{7} \neq 0$ in the following and explore the second possibility, the vanishing of the expression in the parenthesis in (4.17). By solving $\delta g_{6}=0$ we see explicitly that $g_{9} \equiv s_{9}^{3} g_{9}^{Q}=g_{9+3 n_{2}}^{R}$ now take the same form. Solving $\delta g_{6}=0$ e.g. for $s_{6}$ we determine

$$
g_{9}=\frac{1}{2} s_{7} s_{9}^{2}\left(s_{3} s_{8}^{2}-s_{2} s_{8} s_{9}+s_{1} s_{9}^{2}\right) .
$$

Noting that $s_{9} \neq 0$ by assumption, we drop now the coefficients of $s_{7}$ and $s_{9}$ in front of $\delta g_{6}$ and $g_{9}$. Then the codimension two loci for matter read

$$
\delta g_{6}^{\prime}:=s_{7} s_{8}^{2}+s_{9}\left(-s_{6} s_{8}+s_{5} s_{9}\right)=0, \quad g_{9}^{\prime}:=s_{3} s_{8}^{2}-s_{2} s_{8} s_{9}+s_{1} s_{9}^{2}=0 .
$$

To check the order of vanishing of the discriminant, cf. appendix A and section 3.1, along the codimension two locus we consider a small neighborhood around (4.14) and (4.15) defined as

$$
\delta g_{6}^{\prime}=\epsilon, \quad g_{9}^{\prime}=\epsilon, \quad s_{9} \neq 0, \quad s_{7} \neq 0 .
$$

for a small $\epsilon>0$. Again by solving for two of the $s_{i}$ coefficients we see that the discriminant factors as

$$
\Delta=\epsilon^{2} \Delta^{\prime},
$$

with $\Delta^{\prime} \neq 0$ in the limit $\epsilon \rightarrow 0$, which is equivalent to the observation $\hat{g}_{12}^{Q} \sim \hat{g}_{12+4 n_{2}}^{R}=0$ if 4.20 is obeyed. This is an indication for an $I_{2}$ singularity at $\epsilon=0$. Indeed, by solving the conditions (4.20) for $s_{3}$ and $s_{6}$ and by plugging back into (3.4) on the resolution, we obtain a split of the elliptic curve as $\mathcal{E}=c_{1}+c_{2}$ with

$$
\begin{array}{ll}
c_{1}: & s_{8} u+s_{9} v=0 . \\
c_{2}: & s_{1} s_{8} s_{9} u^{2}+s_{2} s_{8} s_{9} u v-s_{1} s_{9}^{2} u v+s_{5} s_{8} s_{9} u w+s_{7} s_{8}^{2} v w+s_{8}^{2} s_{9} w^{2}=0 .
\end{array}
$$

From this we see that the zero section $\hat{s}_{P}=[0: 0: 1]$ intersects $c_{1}$ at a point and does not intersect $c_{2}$, while the other two sections, $\hat{s}_{Q}=[0: 1: 0]$ and $\hat{s}_{R}=\left[0: s_{9},-s_{7}\right]$, intersect $c_{2}$ at a point but not $c_{1}$. This implies as before that the curve $c_{1}$ is the proper transform of the original singular curve under the birational map (3.8) while the curve $c_{2}$ is blown down to a point. It is the isolated rational curve and contributes a hypermultiplet of charges $\left(q_{1}, q_{2}\right)=(1,1)$ according to 2.20 .

\subsection{Singular Rational Sections: charges $(-1,1),(-1,-2),(0,2)$}

There is another way of looking for singularities of the elliptic fibration, that contribute charged matter in F-theory, which is the third type mentioned at the beginning of this 
section. Qualitatively, the strategy is as follows. For non-trivial sections $s_{9}$ and $s_{7}$ over $B$, the section $\hat{s}_{R}=\left[0:-s_{9}: s_{7}\right]$ can 'move' on the fiber $\mathcal{E}$ when varying the point on the base $B$. This implies that there are loci in $B$ where two or more sections intersect in the fiber $\mathcal{E}$. This is achieved when $s_{7}=0$ or $s_{9}=0$ as can be seen from 3.6$)$ in the $\mathbb{P}^{2}$-model for $\mathcal{E}$, see figure 4 . Plugging this ansatz into the discriminant $\Delta$ admits a factorization.

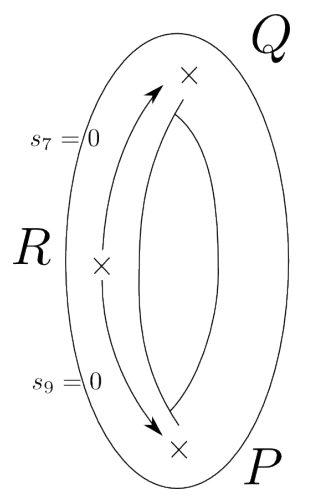

Figure 4: Fibers where two out of the three sections collide. The intersections occur when $s_{7}$ or/and $s_{9}$ vanish on the base $B$.

The results of this analysis, that are precisely the loci not discussed in section 4.2, are summarized in table 4.2 .

\begin{tabular}{|c|c|c|}
\hline Locus & Isolated Curve & Charge $\left(U(1)_{Q}, U(1)_{R}\right)$ \\
\hline$s_{3}=s_{7}=0$ & $e_{1}=0$ & $(-1,1)$ \\
$s_{7}=s_{9}=0$ & $u=0$ & $(0,2)$ \\
$s_{8}=s_{9}=0$ & $c_{2}$ & $(-1,-2)$ \\
\hline
\end{tabular}

Table 4.2: Codimension two loci with singular behavior of the sections. The identifications of the isolated curves are explained in (4.27), 4.30) and 4.34).

We emphasize that at each of these loci one section $\hat{s}_{P}, \hat{s}_{Q}, \hat{s}_{R}$ is ill-defined. This can be seen most easily on the resolved model of the elliptic curve $\mathcal{E}$ in $d P_{2}$ introduced in section 3.2 . For the convenience of the reader we recall the relevant properties of the resolved geometry. After resolutions along $u=w=0$ and $u=v=0$ the sections take the values

$$
\hat{s}_{P}=\left[-s_{9}: s_{8}: 1: 1: 0\right], \quad \hat{s}_{Q}=\left[-s_{7}: 1: s_{3}: 0: 1\right], \quad \hat{s}_{R}=\left[0: 1: 1:-s_{7}: s_{9}\right],
$$


cf. (3.28). We also recall the Stanley-Reissner ideal, of the resolution ${ }^{16}$

$$
S R=\left\{u v, u w, e_{1} e_{2}, e_{1} v, e_{2} w\right\} .
$$

Indeed, at each locus in table 4.2 one of the sections 4.24 is ill-defined since it passes through the Stanley-Reissner ideal (4.25). This requires additional blow-ups in the base $\pi_{B}: \hat{B} \rightarrow B$ as advertised in section 2.2 precisely along the loci where the sections are not well-defined. We emphasize that this implies that the birational map (3.8) is not enough to completely understand and resolve the fibration $X$, in contrast to the codimension two loci discussed in sections 4.1 and 4.2 .

To understand what is happening when one of the sections $\hat{s}_{P}, \hat{s}_{Q}, \hat{s}_{R}$, collectively denoted as $\hat{s}$ for the following paragraph, is singular, we first look at the sections at a location in the base where the fiber is not singular. In this case, after performing the blowups in the ambient space to $d P_{2}$, the exceptional $\mathbb{P}^{1}$ 's intersect the Calabi-Yau polynomial at exactly one point in the fiber with coordinates shown in 4.24). At any of the loci of table 4.2, when two coefficients, $s_{i}$ and $s_{j}$ vanish simultaneously, the two coordinates of the exceptional $\mathbb{P}^{1}$ vanish simultaneously and the coordinates stop making sense. This is another way of saying that the section passes through the Stanley-Reissner ideal (4.25). To resolve this problem we blow up the base at this locus by introducing coordinates $\left[\ell_{1}: \ell_{2}\right] \in \mathbb{P}^{1}$ and writing

$$
\ell_{1} s_{i}-\ell_{2} s_{j}=0 .
$$

The coordinates (4.24) of the section $\hat{s}$ will be now given by the point $\left[s_{j}: s_{i}\right]=\left[\ell_{1}: \ell_{2}\right]$ in $\mathbb{P}^{1}$. When the two coordinates vanish simultaneously, $s_{i}=s_{j}=0$, the values of $\ell_{1}$ and $\ell_{2}$ in 4.26 become unrestricted and we obtain the entire $\mathbb{P}^{1}$ in the fiber.

Another way of seeing this is to notice that the section $\hat{s}$ takes different values at the singular locus $s_{i}=s_{j}=0$ depending on the direction (parametrized by $\left[\ell_{1}: \ell_{2}\right]$ ) at which we approach the locus in the base. Coming in all possible directions makes the section take all possible values at the same locus: the section becomes the entire $\mathbb{P}^{1}$ of slopes.

Now that we understand that the sections can become entire $\mathbb{P}^{1}$ 's, we demonstrate the factorization of the equation (3.27) into two rational curves along all codimension two loci in table 4.2. We discuss these loci successively and refer to figure 5 to keep track of which of the three sections wraps a $\mathbb{P}^{1}$ in the resolved fiber.

\footnotetext{
${ }^{16}$ In the following we denote the coordinates on $d P_{2}$ as $\left[u: v: w: e_{1}: e_{2}\right]$ in contrast to section 3.2 .
} 

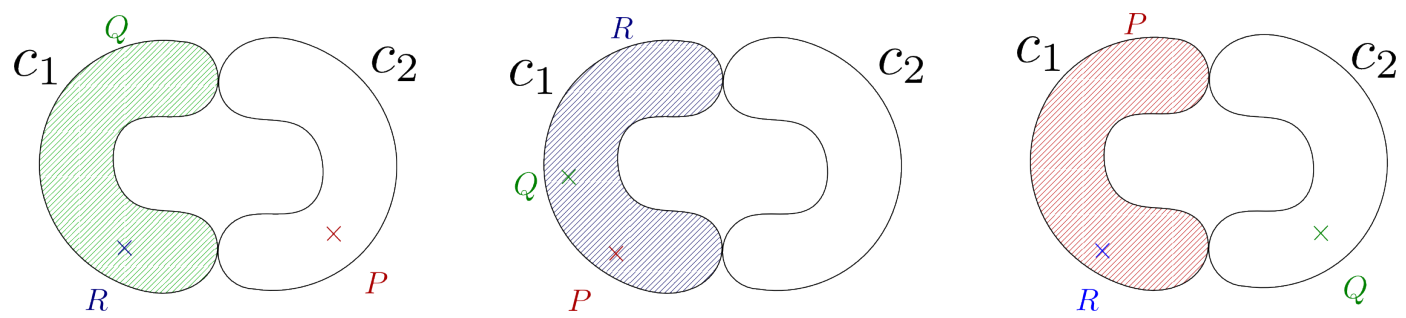

Figure 5: How the fiber degenerates at the three loci $(-1,1),(0,2)$ and $(-1,-2)$ respectively.

Singularity of $\hat{s}_{Q}$ \& blow-up at $s_{3}=s_{7}=0$ : charge $(-1,1)$

As can be seen from (4.24) at the loci $s_{3}=s_{7}=0$ the section $\hat{s}_{Q}$ passes through the ideal (4.25) and is ill-defined. It has to be blown-up and wraps an entire $\mathbb{P}^{1}$-component of the fiber, which is given by the divisor $E_{1}:=\left\{e_{1}=0\right\}$ in $d P_{2}$. In addition, the polynomial (3.27) factorizes into two rational curves, i.e. $\mathcal{E}=c_{1}+c_{2}$, reading

$$
\begin{array}{ll}
c_{1}: & e_{1}=0 \\
c_{2}: & s_{1} e_{1} e_{2}^{2} u^{3}+s_{2} e_{2}^{2} u^{2} v+s_{5} e_{1} e_{2} u^{2} w+s_{6} e_{2} u v w+s_{8} e_{1} u w^{2}+s_{9} v w^{2}=0
\end{array}
$$

We notice using (4.24), 4.25) and the toric intersections (3.25) on $d P_{2}$ the intersections of the rational sections with the two curves as

$$
S_{P} \cdot c_{1}=0, \quad S_{P} \cdot c_{2}=1, \quad S_{Q} \cdot c_{1}=-1, \quad S_{Q} \cdot c_{2}=0, \quad S_{R} \cdot c_{1}=1, \quad S_{R} \cdot c_{2}=0,
$$

where we denoted the homology classes of the sections by capital letters $S_{\bullet}$ as before. Here we note that the last two intersections follow, besides from the toric intersections (3.25), also from the defining property of a section, $1=S_{R} \cdot \mathcal{E}=S_{R} \cdot\left(c_{1}+c_{2}\right)$, which with $S_{R} \cdot c_{2}=0$ immediately yields $S_{R} \cdot c_{1}=1$.

We note that the intersections 4.29 imply that $c_{2}$ is the original singular curve since it intersects the zero section $\hat{s}_{P}$ and $c_{1}$ is the exceptional $\mathbb{P}^{1}$ contributing a hypermultiplet. Thus it follows from 2.20 that the $\mathrm{U}(1)_{Q} \times \mathrm{U}(1)_{R^{-}}$charges are $\left(q_{1}, q_{2}\right)=(-1,1)$.

\section{Singularity of $\hat{s}_{R} \&$ blow-up at $s_{7}=s_{9}=0:$ charge $(0,2)$}

Proceeding in a similar way as before we see that the section $\hat{s}_{R}$ is blown up in a $\mathbb{P}^{1}$, which we identify with the toric divisor $D_{u}:=\{u=0\}$ in $d P_{2}$. Furthermore, the polynomial 
(3.27) factorizes at $s_{7}=s_{9}=0$ into two rational curves given by

$$
\begin{array}{ll}
c_{1}: & u=0 \\
c_{2}: & s_{1} e_{1}^{2} e_{2}^{2} u^{2}+s_{2} e_{1} e_{2}^{2} u v+s_{3} e_{2}^{2} v^{2}+s_{5} e_{1}^{2} e_{2} u w+s_{6} e_{1} e_{2} v w+s_{8} e_{1}^{2} w^{2}=0
\end{array}
$$

The intersections of the sections are evaluated as before employing (4.24), (4.25) and the toric intersections 3.25 on $d P_{2}$ as

$$
S_{P} \cdot c_{1}=1, \quad S_{P} \cdot c_{2}=0, \quad S_{Q} \cdot c_{1}=1, \quad S_{Q} \cdot c_{2}=0, \quad S_{R} \cdot c_{1}=-1, \quad S_{R} \cdot c_{2}=2 .
$$

Here we have determined the intersections of $S_{R}$ by noting that due to the blow-up in the base at $s_{7}=s_{9}=0$, cf. 4.24, it wraps the entire $\mathbb{P}^{1}$ corresponding to the ray subdividing the cone formed $e_{1}, e_{2}$. As we see by recalling figure 2 this is nothing else but the toric divisor with $D_{u}$ section $u$. This then implies that $S_{P} \cdot c_{2}=2$ because the equation for $c_{2}$ has two solutions with $u=0$. From the defining property $1=S_{P} \cdot \mathcal{E}=S_{P} \cdot\left(c_{1}+c_{2}\right)=$ $S_{P} \cdot c_{1}+2$ we infer $S_{P} \cdot c_{1}=-1$ as claimed in (4.32).

From the location of the zero section we infer that $c_{1}$ is the original singular fiber and $c_{2}$ the isolated rational curve contributing a hypermultiplet. Its charges are calculated employing 2.20 as $\left(q_{1}, q_{2}\right)=(0,2)$.

\section{Singularity of $\hat{s}_{P} \&$ blow-up at $s_{8}=s_{9}=0$ : charge $(-1,-2)$}

At this locus, the zero section $\hat{s}_{P}$ is ill-defined and is blown up into a rational fiber component. It is identified with the toric divisor $E_{2}:=\left\{e_{2}=0\right\}$ in $d P_{2}$. The constraint (3.27) of the elliptic curve implies a split $\mathcal{E}=c_{1}+c_{2}$ at these loci with

$$
\begin{array}{ll}
c_{1}: & e_{2}=0 \\
c_{2}: & s_{1} e_{1}^{2} e_{2} u^{3}+s_{2} e_{1} e_{2} u^{2} v+s_{3} e_{2} u v^{2}+s_{5} e_{1}^{2} u^{2} w+s_{6} e_{1} u v w+s_{7} v^{2} w=0 .
\end{array}
$$

As before the intersections of the sections are computed from 4.24), 4.25 and the toric intersections (3.25) on $d P_{2}$ as

$$
S_{P} \cdot c_{1}=-1, \quad S_{P} \cdot c_{2}=2, \quad S_{Q} \cdot c_{1}=0, \quad S_{Q} \cdot c_{2}=1, \quad S_{R} \cdot c_{1}=1, \quad S_{R} \cdot c_{2}=0,
$$

where the intersection $S_{P} \cdot c_{2}=2$ can be obtained by setting $e_{2}=0$ in (4.34). Alternatively, it can also be calculated using $1=S_{P} \cdot \mathcal{E}=S_{P} \cdot\left(c_{1}+c_{2}\right)=-1+S_{P} \cdot c_{2}$. 
Here we have used that the blow-up in the base along $s_{8}=s_{9}=0$ has blown-up the zero section with coordinates (4.24) into $E_{2}$, which is the toric divisor of the ray subdividing the cone spanned by $u$ and $v$. The curve $c_{1}$ is part of the zero section $c_{1}$, thus it is the original singular fiber, whereas $c_{2}$ maps to the singular point of the original fiber. Thus $c_{2}$ is the isolated curve and it contributes according to (2.20) a hypermultiplet of total charge $\left(q_{1}, q_{2}\right)=(-1,-2)$. We note that this locus is special in the sense that it supports the only matter multiplet where the second term in $(2.20)$ contributes to the charge due to the ill-defined zero section $\hat{s}_{P}$.

\subsection{Calculating Matter Multiplicities}

Now that we have determined the full matter representations and their codimension two loci in the base $B$, we can count their multiplicities. The main complication that arises in these calculations is due to the intersection of different codimension two loci. In order to avoid double-counting we thus have to appropriately subtract the number of intersection points of a codimension two loci under consideration with other codimension two loci.

For this analysis, we first have to recall the counting of the multiplicity of a root in a system of two polynomials with two independent variables. Given two polynomials

$$
f(x, y)=0, \quad g(x, y)=0,
$$

we define

$$
h(y):=\operatorname{Res}_{x}(f, g)
$$

as the resultant of $f, g$ with respect to $x$. The degree of a root $\left(x_{0}, y_{0}\right)$ of 4.36 is given by the multiplicity of $y_{0}$ as a zero of $h(y)$ 17

Now that we know how to count roots of a polynomial system, the strategy is simple: take the degrees of the polynomials for the codimension two loci we are interested, multiply them and then subtract all intersecting loci we are not interested in with the appropriate multiplicity. As a warm up and a demonstration of this technique we rederive the multiplicity of the charge one loci of the elliptic fibration over $\mathbb{P}^{2}$ with elliptic fiber $B l_{(0,1,0)} \mathbb{P}^{1,1,2}$ considered in [43].

\footnotetext{
${ }^{17}$ The degree can also be defined with respect to the variable $x$, however, yielding the same multiplicity.
} 


\section{Example: Charge one multiplicity of the $B l_{(0,1,0)} \mathbb{P}^{2}(1,1,2)$ model}

The loci of the charge one hypermultiplets were given in equations (5.64) of [43]

$$
\begin{aligned}
f_{3+n}^{3}-3 f_{6} f_{3+n} b_{n}^{2}+b_{n}^{4} f_{9-n} & =0, \\
f_{3+n}^{4}-6 f_{6} f_{3+n}^{2} b_{n}^{2}+9 f_{6}^{2} b_{n}^{4}-f_{12-2 n} b_{n}^{6} & =0,
\end{aligned}
$$

with $b_{n} \neq 0$ and $f_{3+n} \neq 0$ because those were identified as the charge two loci. We see that $f_{3+n}=b_{n}=0$ obviously solves the system.

Viewing now the equations (4.38) as polynomials in the variables $f_{3+n}$ and $b_{n}$, the resultant with respect to the variable $f_{3+n}$ is given by

$$
h\left(b_{n}\right)=b_{n}^{16}\left(b_{n}^{2} f_{12-2 n}^{3}-9 f_{12-2 n}^{2} f_{6}^{2}+6 f_{12-2 n} f_{6} f_{9-n}^{2}-f_{9-n}^{4}\right),
$$

from where it can be seen that $f_{3+n}=b_{n}=0$ is a root of degree 16 of the system (4.38). Now we can calculate the multiplicity of the charge one hypermultiplets. The degrees of (4.38) are $(9+3 n)$ and $(12+4 n)$ respectively and there are $n(3+n)$ roots we are not interested in and that have to be subtracted, each of which of degree 16. Thus we obtain

$$
(9+3 n)(12+4 n)-16 n(3+n)=4(9-n)(3+n)
$$

charge one hypermultiplets.

\section{Matter multiplicities of the $d P_{2}$ model}

In this subsection we will finally apply the resultant technique to determine the multiplicity of all six codimension two matter loci found in sections 4.1, 4.2 and 4.3. We denote the multiplicities by the same variables $x_{i}, i=1, \ldots, 6$, as in (5.5) to facilitate a straightforward comparison with results from anomaly cancellation.

The basis of our analysis are the degrees of the coefficients $s_{i}$ calculated in 3.32 for the base $B=\mathbb{P}^{2}$. Let us recall that the degree of $s_{9}$ is equal to the intersection number $n_{2}$ of the zero section $\hat{s}_{P}$ and the section $\hat{s}_{R}$ and the degree $n_{12}$ of $s_{7}$ equals the intersection number of $\hat{s}_{Q}$ and $\hat{s}_{R}$, cf. (3.33). With these degrees, the multiplicities of the hypermultiplets of section 4.3 are straightforward: they are simply the multiplication of the degrees of the two coefficient that vanish, see table 4.3 . For the other hypermultiplets we have to work a little more. 


\begin{tabular}{|c|c|c|}
\hline Locus & Charge $\left(U(1)_{Q}, U(1)_{R}\right)$ & Multiplicity \\
\hline$s_{3}=s_{7}=0$ & $(-1,1)$ & $x_{4}=\left(3-n_{2}+n_{12}\right) n_{12}$ \\
$s_{7}=s_{9}=0$ & $(0,2)$ & $x_{5}=n_{12} n_{2}$ \\
$s_{8}=s_{9}=0$ & $(-1,-2)$ & $x_{6}=\left(3+n_{2}-n_{12}\right) n_{2}$ \\
\hline
\end{tabular}

Table 4.3: Matter multiplicities.

\section{Charge $(1,1)$}

The charge $(1,1)$ hypermultiplets are located at the roots of equations $(4.19)$, that we recall for convenience of the reader as

$$
\delta g_{6}^{\prime}=s_{7} s_{8}^{2}+s_{9}\left(-s_{6} s_{8}+s_{5} s_{9}\right) \stackrel{!}{=} 0, \quad g_{9}^{\prime}=s_{3} s_{8}^{2}-s_{2} s_{8} s_{9}+s_{1} s_{9}^{2} \stackrel{!}{=} 0 .
$$

They have degrees

$$
\begin{aligned}
\operatorname{deg}\left(\delta g_{6}^{\prime}\right) & =n_{12}+2\left(3+n_{2}-n_{12}\right)=6+2 n_{2}-n_{12}, \\
\operatorname{deg}\left(g_{9}^{\prime}\right) & =\left(3-n_{2}+n_{12}\right)+2\left(3+n_{2}-n_{12}\right)=9+n_{2}-n_{12} .
\end{aligned}
$$

The loci $s_{8}=s_{9}=0$ are obviously roots of these equations. The degree of this root is four as we see by working out the resultant of these constraints with respect to $s_{8}$ and $s_{9}$. No other codimension two loci satisfy the equations (4.41). Thus, the multiplicity of the hypermultiplets with charges $(1,1)$ is

$x_{3}=\left(6+2 n_{2}-n_{12}\right)\left(9+n_{2}-n_{12}\right)-4 n_{2}\left(3+n_{2}-n_{12}\right)=54+12 n_{2}-2 n_{2}^{2}+\left(n_{2}-15\right) n_{12}+n_{12}^{2}$

\section{Charge $(1,0)$}

We proceed with the calculation of the multiplicity of charge $(1,0)$ hypermultiplets. The constraints of the corresponding codimension two loci is given by equation (4.6), that takes the schematic form

$$
g_{9}^{Q}=0, \quad \hat{g}_{12}^{Q}=0 .
$$

Recalling table 4.2 we notice that all hypermultiplets with charges $(1,1),(-1,1)$ and $(-1,-2)$ satisfy these equations. The multiplicity is calculated from the resultant as one 
in each case. Thus, the multiplicity of the loci supporting charge $(1,0)$ hypermultiplets is then

$$
\begin{aligned}
x_{1} & =108-\left(54+12 n_{2}-2 n_{2}^{2}+\left(n_{2}-15\right) n_{12}+n_{12}^{2}\right)-n_{12}\left(3-n_{2}+n_{12}\right)-n_{2}\left(3+n_{2}-n_{12}\right) \\
& =54-15 n_{2}+n_{2}^{2}+12 n_{12}+n_{2} n_{12}-2 n_{12}^{2} .
\end{aligned}
$$

\section{Charge $(0,1)$}

The defining equations for the loci supporting these hypermultiplets are given by the equations in (4.10), that schematically read as

$$
g_{9+3 n_{2}}^{R}=0, \quad \hat{g}_{12+4 n_{2}}^{R}=0 .
$$

Again by looking at table 4.2 we see that all hypermultiplets with charge $(1,1),(-1,1)$, $(0,2)$ and $(-1,-2)$ satisfy the equations with multiplicities. Their multiplicities are 1 , 1,16 and 16, respectively, and by subtracting from the degree of 4.45 we obtain the multiplicity as

$$
\begin{aligned}
x_{2} & =\left(9+3 n_{2}\right)\left(12+4 n_{2}\right)-x_{3}-x_{4}-16 x_{5}-16 x_{6} \\
& =54+12 n_{2}-2 n_{2}^{2}+12 n_{12}-2 n_{12}^{2} .
\end{aligned}
$$

\section{Anomaly Cancellation: a Consistency Check}

In this section we check the consistency of the matter spectrum determined in the previous section via constraints arising from anomaly cancellation. We demonstrate that anomaly cancellation allows us to classify all F-theory compactifications with base $B=\mathbb{P}^{2}$ and the two section elliptic fiber determined in section 3.1. This confirms the geometric analysis of the previous section that has led to determination of the charges of matter fields. We also see that the computed multiplicities agree with the ones predicted by anomaly cancellation. This analysis ensure a consistent low-energy theory of F-theory in six dimensions with two Abelian gauge fields.

Anomaly cancellation in six-dimensional F-theory vacua, based on general results on 6d anomaly cancellation [59, 60], has been discussed in [55, 43], whose notations and conventions we follow here. See also the analysis in [44] of anomaly cancellation in four 
dimensional F-theory compactifications. We note that the following discussion holds more generally for any Abelian gauge theory.

We are interested in anomaly cancellation in an Abelian gauge theory with matter arising from F-theory compactifications. In this case, there are only the purely gravitational anomaly, the mixed Abelian-gravitational and the pure Abelian gauge anomalies. We assume that there are $H$ hypermultiplets, $T$ tensor multiplets and $V$ vector multiplets. The gauge and mixed anomalies induced by the six-dimensional charged matter in hypermultiplets have to be canceled by a classical effect, the generalized Green-Schwarz mechanism [61]. These cancellation conditions are the anomaly equations. The mixed Abelian-gravitational, purely Abelian and purely gravitational anomaly equations read

$$
\begin{aligned}
K_{B} \cdot b_{m n} & =-\frac{1}{6} \sum_{\underline{q}} x_{q_{m}, q_{n}} q_{m} q_{n}, \\
b_{m n} \cdot b_{k l}+b_{m k} \cdot b_{n l}+b_{m l} \cdot b_{n k}= & \sum_{\underline{q}} x_{q_{m}, q_{n}, q_{k}, q_{l}} q_{m} q_{n} q_{k} q_{l}, \\
273=H-V+29 T, & K_{B} \cdot K_{B}=9-T .
\end{aligned}
$$

Here we have labeled the $r \mathrm{U}(1)$-fields in our theory by $A^{m}$, where $r$ denotes the rank of the Mordell-Weil group of the Calabi-Yau threefold $X$. On the right hand side of (5.1), the sum runs over all hypermultiplets with charge vector $q=\left(q_{1}, \ldots, q_{r}\right)$. The integers $x_{q_{m}, q_{n}}$ respectively $x_{q_{m}, q_{n}, q_{k}, q_{l}}$ denote the number of hypermultiplets with charges $\left(q_{m}, q_{n}\right)$ under $\mathrm{U}(1)_{m} \times \mathrm{U}(1)_{n}$ respectively charges $\left(q_{m}, q_{n}, q_{k}, q_{l}\right)$ under $\mathrm{U}(1)_{m} \times \mathrm{U}(1)_{n} \times \mathrm{U}(1)_{k} \times \mathrm{U}(1)_{l}$. The left hand side of (5.1) is to be evaluated in the cohomology of the base $B$ of the fibration. $K_{B}$ denotes the canonical class of $B$, - is the intersection pairing on $B$ and the expressions $b_{m n}$ are defined as the curves

$$
\begin{aligned}
b_{m n} & =-\pi\left(\sigma\left(\hat{s}_{m}\right) \cdot \sigma\left(\hat{s}_{n}\right)\right) \\
& =-\pi\left(S_{m} \cdot S_{n}\right)-\left[K_{B}\right]+\pi\left(S_{m} \cdot S_{P}\right)+\pi\left(S_{n} \cdot S_{P}\right) .
\end{aligned}
$$

We recall that $S_{m}$ denotes the divisors class of a section $\hat{s}_{m}$ and the homology class $S_{P}$ of the zero section $\hat{s}_{P}$. Here the first line has been evaluated employing (2.16) in the presence of only Abelian gauge fields. We note that the pure gravitational anomaly even without further specification of the spectrum puts an upper bound on a theory with $T=0$, which is the case for $B=\mathbb{P}^{2}$, as

$$
\sum_{f} N_{f} \leq 275 .
$$


We now analyze the anomaly constraints (5.1) for the F-theory compactification on the elliptic fibration over $\mathbb{P}^{2}$ with two U(1) gauge fields and the matter content determined in section 4. We see that anomaly cancellation fixes the possible matter multiplicities in terms of two integers. We first evaluate the anomaly coefficients $b_{m n}$ in (5.1). In the case of $\mathbb{P}^{2}$ as the base, there is only one element generating $H_{2}(B)$, the hyperplane class $H_{B}$. The anti-canonical line bundle is $K_{\mathbb{P}^{2}}=-3 H_{B}$. Then the coefficients are just numbers, namely the coefficients of $H_{B}$, and evaluated to

$$
b_{k l}=\left\{\begin{array}{cc}
2\left(n_{k}+3\right), & k=l \\
3+n_{k}+n_{l}-n_{k l}, & k \neq l,
\end{array}\right.
$$

where we defined $n_{k l}=\pi\left(S_{k} \cdot S_{l}\right)$ and $n_{k}=\pi\left(S_{k} \cdot B\right)$. In addition, we have employed the second relation in (2.11) to replace $\pi\left(S_{k} \cdot S_{k}\right)=\left(S_{k} \cdot S_{k} \cdot H_{B}\right) H_{B}=K=3 H_{B}$. Then, we evaluate the right hand side of (5.1) using the spectrum found in section 4 with arbitrary multiplicities $x_{i}$ assigned as

\begin{tabular}{|c||c|c|c|c|c|c|}
\hline$\left(q_{1}, q_{2}\right)$ & $(1,0)$ & $(0,1)$ & $(1,1)$ & $(-1,1)$ & $(0,2)$ & $(-1,-2)$ \\
\hline multiplicity & $x_{1}$ & $x_{2}$ & $x_{3}$ & $x_{4}$ & $x_{5}$ & $x_{6}$ \\
\hline
\end{tabular}

Upon inserting this into the anomaly constraints (5.1) for two sections $S_{1} \equiv S_{Q}, S_{2} \equiv S_{R}$ and the zero section $S_{P}$ we obtain a set of linear equations for the $x_{i}$ reading

$$
\begin{gathered}
36\left(3+n_{1}\right)=12\left(3+n_{1}\right)^{2}=x_{1}+x_{3}+x_{4}+x_{6}, \\
18\left(3+n_{1}-n_{12}+n_{2}\right)=6\left(3+n_{1}\right)\left(3+n_{1}-n_{12}+n_{2}\right)=x_{3}-x_{4}+2 x_{6}, \\
12\left(3+n_{2}\right)^{2}=x_{2}+x_{3}+x_{4}+16 x_{5}+16 x_{6}, \\
6\left(3+n_{2}\right)\left(3+n_{1}-n_{12}+n_{2}\right)=x_{3}-x_{4}+8 x_{6}, \\
36\left(3+n_{2}\right)=x_{2}+x_{3}+x_{4}+4 x_{5}+4 x_{6}, \\
4\left(3+n_{1}\right)\left(3+n_{2}\right)+2\left(3+n_{1}-n_{12}+n_{2}\right)^{2}=x_{3}+x_{4}+4 x_{6} .
\end{gathered}
$$

We see that the first equation in this system immediately requires $n_{1}=\pi\left(S_{Q} \cdot S_{P}\right)=0$. This nicely agrees with the finding of (3.11) respectively (3.28) that the section $S_{Q}$ does not intersect the zero section $S_{P}$. The solutions of (5.6) then take the form

$$
\begin{array}{ll}
x_{1}=54-15 n_{2}+n_{2}^{2}+\left(12+n_{2}\right) n_{12}-2 n_{12}^{2}, & x_{4}=n_{12}\left(3-n_{2}+n_{12}\right), \\
x_{2}=54+2\left(6 n_{2}-n_{2}^{2}+6 n_{12}-n_{12}^{2}\right), & x_{5}=n_{2} n_{12}, \\
x_{3}=54+12 n_{2}-2 n_{2}^{2}+\left(n_{2}-15\right) n_{12}+n_{12}^{2}, & x_{6}=n_{2}\left(3+n_{2}-n_{12}\right) .
\end{array}
$$


This provides a full classification of the possible matter multiplicities with two U(1)-gauge fields in terms of the intersections

$$
n_{2}=\pi\left(S_{Q} \cdot S_{P}\right), \quad n_{12}=\pi\left(S_{Q} \cdot S_{R}\right)
$$

of the two sections $S_{Q}, S_{R}$ and of $S_{P}$ introduced in (2.17). It is satisfying that the matter multiplicities found in section 4.4 are reproduced by (5.7).

We conclude by evaluating two special cases. First we consider $n_{2}=n_{12}=0$ where we obtain

\begin{tabular}{|c||c|c|c|c|c|c|}
\hline$\left(q_{1}, q_{2}\right)$ & $(1,0)$ & $(0,1)$ & $(1,1)$ & $(-1,1)$ & $(0,2)$ & $(-1,-2)$ \\
\hline multiplicity & 54 & 54 & 54 & 0 & 0 & 0 \\
\hline
\end{tabular}

In this case, charge two loci are completely absent. A less trivial example can be constructed by setting $n_{2}=n_{12}=1$ in which case we obtain

\begin{tabular}{|c||c|c|c|c|c|c|}
\hline$\left(q_{1}, q_{2}\right)$ & $(1,0)$ & $(0,1)$ & $(1,1)$ & $(-1,1)$ & $(0,2)$ & $(-1,-2)$ \\
\hline multiplicity & 51 & 74 & 51 & 3 & 1 & 3 \\
\hline
\end{tabular}

The global geometries of both examples are constructed in section 6. As we see there, we will find perfect agreement with results from anomaly cancellation by using the general geometric techniques of section 4.

\section{Toric Elliptic Calabi-Yau Manifolds with Two Rational Sections}

In this section we construct explicitly toric elliptically fibered Calabi-Yau threefolds realizing concrete elliptic fibrations with two rational sections. In all examples the base of the fibration is $B=\mathbb{P}^{2}$ and the generic fiber is the elliptic curve in $d P^{2}$. The first two examples both have $\mathrm{U}(1) \times \mathrm{U}(1)$-gauge symmetry, but with rational sections of different complexity and consequently different matter sectors. In a third example we add an SU(5)-GUT sector and explicitly present the corresponding four-dimensional toric polytope.

In all examples considered below, we construct the toric ambient variety $V$ as a toric fibration of $d P_{2}$ over a toric base $B$,

$$
d P_{2} \rightarrow V \rightarrow B .
$$


The presence of a toric fibration can be detected by analyzing the toric polytope $\Delta_{V}$ defining $V$. The polytope $\Delta_{d P_{2}}$ of the fiber has to be cut out by a two-dimensional plane $E$ through the origin 0 as

$$
\Delta_{d P_{2}}=E \cap \Delta_{V} .
$$

Then a toric morphism $\varphi$ to the base $B$ is defined by first finding the two normal vectors $n_{i}, i=1,2$, to $E$ and then by defining the $2 \times 4$-matrix

$$
\varphi=\left(\begin{array}{c}
n_{1}^{T} \\
\vdots \\
n_{k-1}^{T}
\end{array}\right)
$$

This matrix acts as $\varphi^{T} \cdot p$ on the integral points $p$ of the polytope $\Delta_{V}$ yielding all the integral points of the polytope $\Delta_{B}$ of the base $B$. We denote this in the suggestive form

$$
\Delta_{B}=\varphi^{T} \cdot \Delta .
$$

\subsection{Example 1}

The first and simplest example we consider has three non-intersecting sections. As we have seen in section 4 even in this simple case, matter with charges $(1,0),(0,1)$ and $(1,1)$ can exist and we check explicitly that this is the case. The vertices of the polytope along with their divisor classes and charge vectors $\ell^{(i)}$ read:

\begin{tabular}{|c||c|c|c|c||c||c|c|c|c|}
\hline \multicolumn{1}{|c||}{ variable } & \multicolumn{3}{c||}{ vertices } & divisor class & $\ell^{(0)}$ & $\ell^{(1)}$ & $\ell^{(2)}$ & $\ell^{(3)}$ \\
\hline$z_{0}$ & 1 & 1 & 1 & 0 & $H_{B}$ & 1 & 0 & 0 & 0 \\
$z_{1}$ & -1 & 0 & 1 & 0 & $H_{B}$ & 1 & 0 & 0 & 0 \\
$z_{2}$ & 0 & -1 & 1 & 0 & $H_{B}$ & 1 & 0 & 0 & 0 \\
\hline$u$ & 0 & 0 & 1 & 0 & $-3 H_{B}+H-E_{1}-E_{2}$ & -3 & 1 & 1 & -1 \\
$v$ & 0 & 0 & 0 & 1 & $H-E_{2}$ & 0 & 1 & 0 & 0 \\
$w$ & 0 & 0 & -1 & -1 & $H-E_{1}$ & 0 & 0 & 1 & 0 \\
$e_{1}$ & 0 & 0 & 0 & -1 & $E_{1}$ & 0 & 0 & -1 & 1 \\
$e_{2}$ & 0 & 0 & 1 & 1 & $E_{2}$ & 0 & -1 & 0 & 1 \\
\hline
\end{tabular}

We readily check by the condition (6.2) that the polytope 6.5 describes indeed a toric fibration with the hyperplane $E=\{(0,0, x, y) \mid x, y \in \mathbb{R}\}$. In other words, all points of the form $(0,0, x, y)$ describe the polytope of $d P_{2}$, which are the fourth to eigth points 
in (6.5). The toric morphism $\varphi$ in (6.3) is then constructed as the projection onto the first two coordinates of the vertices and reveals the polytope of the base $\mathbb{P}^{2}$ as the first three points in (6.5). The toric Calabi-Yau hypersurface $\hat{X}$ in the toric variety $V$ specified by (6.5) has Euler number and Hodge numbers

$$
\chi(\hat{X})=-216, \quad h^{(1,1)}(\hat{X})=4, \quad h^{(2,1)}(\hat{X})=112 .
$$

It is satisfying to note that there are neither non-toric divisors nor non-toric complex structure deformations in $\hat{X}$ as expected by construction.

This model is characterized by $n_{2}=n_{12}=0$ as can be seen for example by calculating the toric intersections (3.33). Using the general formula 3.32 or by constructing explicitly the sections of the anti-canonical bundle of (6.5) we can readily determine the coefficients $s_{i}$. They are the most generic polynomials in the projective coordinates $\left[z_{0}: z_{1}: z_{2}\right]$ on $\mathbb{P}^{2}$ of the following degrees or line bundles (LB):

\begin{tabular}{|c|c|}
\hline coefficients & section of LB \\
\hline$s_{9}, s_{7}$ & $\mathcal{O}(0)$ \\
$s_{8}, s_{6}, s_{3}$ & $\mathcal{O}\left(3 H_{B}\right)$ \\
$s_{5}, s_{2}$ & $\mathcal{O}\left(6 H_{B}\right)$ \\
$s_{1}$ & $\mathcal{O}\left(9 H_{B}\right)$ \\
\hline
\end{tabular}

The three sections are given by the intersection of the Calabi-Yau hypersurface with the three toric divisors $D_{u}:=\{u=0\}, E_{1}$ and $E_{2}$. We summarize this schematically as

$$
\hat{s}_{P}:\left\{e_{2}=0\right\} \cap p \quad \hat{s}_{Q}:\left\{e_{1}=0\right\} \cap p, \quad \hat{s}_{R}:\{u=0\} \cap p,
$$

where $p$ is the polynomial of the Calabi-Yau hypersurface. All three sections intersect the fiber once and are holomorphic everywhere on the base $B=\mathbb{P}^{2}$. To be consistent with the notation in the rest of this work, we choose $\hat{s}_{P}$ as the zero section. The other two sections $\hat{s}_{Q}$ and $\hat{s}_{R}$ generate the rank two Mordell-Weill group, responsible for the Abelian $U(1) \times U(1)$-sector of the theory.

The matter content of this example can be determined explicitly in this toric example or by using the results of sections 4. We summarized our findings in equation (5.9) and emphasize that anomaly cancellation is automatic.

\subsection{Example 2}

In order to create a concrete geometry with more complicated rational sections that in particular realize all the matter content found in section 4 it is necessary to fiber the 
$d P_{2}$-polytope slightly differently over the base $\mathbb{P}^{2}$. This requires a change of the line bundle on the base $B$ under which the coordinates $\left[u: v: w ; e_{1}: e_{2}\right]$ transform. A simple way to realize this torically is by changing the location of the base polytope $\Delta_{B}$ in the polytope of the toric fibration as:

\begin{tabular}{|c||c|c|c|c|}
\hline \multicolumn{1}{|c||}{ variable } & \multicolumn{4}{c|}{ vertices } \\
\hline$z_{0}$ & 1 & 1 & 1 & 0 \\
$z_{1}$ & -1 & 0 & 0 & 0 \\
$z_{2}$ & 0 & -1 & 1 & 0 \\
\hline
\end{tabular}

The remaining points take the same form as in 6.5 of the previous example. Thus, the only change of the charge vectors occurs for $\ell^{(0)}$, whose fourth entry -3 is to be replaced by -2 . This means that the coordinate $u$ now transforms in the bundle $\mathcal{O}_{\mathbb{P}^{2}}(-2)$ over $\mathbb{P}^{2}$.

As before we check the existence of an elliptic fibration by the condition (6.2) with the hyperplane $E=\{(0,0, x, y) \mid x, y \in \mathbb{R}\}$. The toric morphism $\varphi$ in 6.3 is constructed as before as the projection onto the first two coordinates of the vertices. The toric CalabiYau hypersurface $\hat{X}$ in the toric variety $V$ specified by $(6.9)$ has Euler number and Hodge numbers

$$
\chi(\hat{X})=-174, \quad h^{(1,1)}(\hat{X})=4, \quad h^{(2,1)}(\hat{X})=91,
$$

and again there are neither non-toric divisors nor non-toric complex structure deformations in $\hat{X}$ as expected.

This model is characterized by $n_{2}=n_{12}=1$ as can be seen for example by calculating the toric intersections (3.33). By means of the general formula $(3.32)$ or by constructing explicitly the sections of its anti-canonical bundle we determine the coefficients $s_{i}$ that are the most generic polynomials in the projective coordinates $\left[z_{0}: z_{1}: z_{2}\right]$ on $\mathbb{P}^{2}$ of the following degrees:

\begin{tabular}{|c|c|}
\hline coefficients & section of LB \\
\hline$s_{9}, s_{7}$ & $\mathcal{O}\left(H_{B}\right)$ \\
$s_{8}, s_{6}, s_{3}$ & $\mathcal{O}\left(3 H_{B}\right)$ \\
$s_{5}, s_{2}$ & $\mathcal{O}\left(5 H_{B}\right)$ \\
$s_{1}$ & $\mathcal{O}\left(7 H_{B}\right)$ \\
\hline
\end{tabular}

The three rational sections are given by the intersection of the toric divisors with the Calabi-Yau hypersurface $p=0$ as

$$
\hat{s}_{P}:\left\{e_{2}=0\right\} \cap p \quad \hat{s}_{Q}:\left\{e_{1}=0\right\} \cap p, \quad \hat{s}_{R}:\{u=0\} \cap p,
$$


Since $n_{2}=n_{12}=1$ there are loci where each of the sections $\hat{s}_{P}, \hat{s}_{Q}$ and $\hat{s}_{R}$ are illdefined, cf. table4.2. Thus, the full spectrum analyzed in section 4 is realized. The matter multiplicities follow readily from the classification in section 4.4 and were calculated explicitly in (5.10). Again, it is seen that anomalies are canceled.

\subsection{Example with SU(5)}

In this concluding example we construct a concrete resolved elliptically fibered CalabiYau threefold over $\mathbb{P}^{2}$ with an $S U(5)$-GUT sector. The geometry constructed below is an extension of the geometry constructed in section 6.1 by a resolved SU(5)-singularity on the divisor $z_{2}=0$. The full gauge symmetry of the F-theory compactification is thus $\mathrm{SU}(5) \times \mathrm{U}(1) \times \mathrm{U}(1)$.

The variables, vertices of the polytope describing the toric variety $V$ and the independent toric divisor classes read:

\begin{tabular}{|c||c|c|c|c||c|}
\hline \multicolumn{1}{|c||}{ variable } & \multicolumn{4}{|c||}{ vertices } & divisor class \\
\hline$z_{0}$ & 1 & 1 & 1 & 0 & $H_{B}$ \\
$z_{1}$ & -1 & 0 & 1 & 0 & $H_{B}$ \\
$z_{2}$ & 0 & -1 & 1 & 0 & $H_{B}-D_{1}-D_{2}-D_{3}-D_{4}$ \\
\hline$d_{1}$ & 0 & -1 & 0 & 0 & $D_{1}$ \\
$d_{2}$ & 0 & -1 & 0 & -1 & $D_{2}$ \\
$d_{3}$ & 0 & -1 & -1 & -2 & $D_{3}$ \\
$d_{4}$ & 0 & -1 & -1 & -1 & $D_{4}$ \\
\hline$u$ & 0 & 0 & 1 & 0 & $-3 H_{B}+H-D_{2}-D_{3}-E_{1}-E_{2}$ \\
$v$ & 0 & 0 & 0 & 1 & $H-D_{1}-D_{2}-D_{3}-D_{4}-E_{2}$ \\
$w$ & 0 & 0 & -1 & -1 & $H-D_{1}-2 D_{2}-3 D_{3}-2 D_{4}-E_{1}$ \\
$e_{1}$ & 0 & 0 & 0 & -1 & $E_{1}$ \\
$e_{2}$ & 0 & 0 & 1 & 1 & $E_{2}$ \\
\hline
\end{tabular}

We note that the Abelian sector has not changed compared to section 6.1. The sections are still located at the following intersections of the Calabi-Yau hypersurface $p=0$,

$$
\hat{s}_{P}:\left\{e_{2}=0\right\} \cap p \quad \hat{s}_{Q}:\left\{e_{1}=0\right\} \cap p, \quad \hat{s}_{R}:\{u=0\} \cap p .
$$

A constructive way to understand the form of the polytope in $(6.13)$ is to start with the singular geometry and perform the four blow-ups necessary to resolved codimension 
one and two singularities of the fibration following [19, 20, 21. Then all one has to do is to translate the divisor classes back into toric charge vectors and find the vertices realizing these charge vectors as linear relations. This analysis is performed in the following and leads to the polytope in (6.13).

As usual an SU(5)-singularity at codimension one in the base $B$ is engineered by specializing the coefficients in the Calabi-Yau hypersurface equation $p=0$, that is specializing the form presented in (3.27). In the fibration with elliptic fiber $d P_{2}$ this means that we have to consider non-generic sections $s_{i}$ in (3.27). Concretely, we engineer an $\mathrm{SU}(5)$-singularity on the divisor $z_{2}=0$ in $\mathbb{P}^{2}$. One way to realize an $\mathrm{SU}(5)$-singularity is to consider non-generic coefficients $s_{i}$ of the form

$$
s_{1}=z_{2}^{3} s_{1}^{\prime}, \quad s_{2}=z_{2}^{2} s_{2}^{\prime}, \quad s_{3}=z_{2}^{2} s_{3}^{\prime}, \quad s_{5}=z_{2} s_{5}^{\prime},
$$

with all other $s_{i}$ generic. The sections $s_{i}$ have to take values in the bundles (6.7), which determines that $s_{1}^{\prime}, s_{2}^{\prime} s_{3}^{\prime}, s_{5}^{\prime}$ have to be generic sections of the bundles $\mathcal{O}\left(6 H_{B}\right), \mathcal{O}\left(4 H_{B}\right)$, $\mathcal{O}\left(H_{B}\right), \mathcal{O}\left(5 H_{B}\right)$, respectively.

With these definitions we can check explicitly, employing the formulae of section 2.1 for $f, g$ and $\Delta$, that the choice (6.15) realizes an SU(5)-singularity at $z_{2}=0$, i.e. $f, g$ do not vanish whereas $\Delta \sim z_{2}^{5}$ as required by the Kodaira classification for an $\mathrm{SU}(5)$ singularity [16]. In fact, by inserting (6.15) into the discriminant we observe the structure

$$
\Delta=-z_{2}^{5}\left(\beta_{5}^{4} P+z_{2} \beta_{5}^{2}\left(8 \beta_{4} P+\beta_{5} R\right)+z_{2}^{2}\left(16 \beta_{3}^{2} \beta_{4}^{3}+\beta_{5} Q\right)+z_{2}^{3} S+z_{2}^{4} T+z_{2}^{5} U+\mathcal{O}\left(z_{2}^{6}\right)\right)
$$

with the identification

$$
\beta_{5}=s_{6}, \quad P:=P_{1} P_{2} P_{3} P_{4} P_{5}=\left(s_{2} s_{5}-s_{1} s_{6}\right) s_{7}\left(-s_{3} s_{6}+s_{2} s_{7}\right) s_{8}\left(-s_{7} s_{8}+s_{6} s_{9}\right) .
$$

All the other coefficients can be determined similarly but are not shown because we are only interested in the structure at codimension two. We readily see that the form of the discriminant agrees with the expected one from the local model analysis in the literature, compare e.g. to [19] whose notation we follow. However, in a local model the discriminant (6.16) contains only terms up to order five in $z_{2}$. As expected for global models there are higher order terms in $z_{2}$ in (6.16), in this case terms up to $z_{2}^{10}$.

Next we turn to the resolution of the singular elliptic fibration over $z_{2}=0$. A completely smooth elliptic fibration is obtained by four consecutive blow-ups in the ambient space, two of which being ordinary blow-ups and the other two being small resolutions on the Calabi-Yau hypersurface. The first blow-up has to be performed at the codimension 
three locus $w=v=z_{2}=0$ in the ambient space. Upon introducing a new section $d_{1}$ corresponding to one exceptional divisor, we formulate the blow-up relation as

$$
w=d_{1} \tilde{w}, \quad v=d_{1} \tilde{v}, \quad z_{2}=d_{1} \tilde{z}_{2} .
$$

The Calabi-Yau manifold is still singular at $\tilde{w}=u=d_{1}=0$. We have to perform another blow-up and upon introducing a new section $d_{2}$ we obtain

$$
\tilde{w}=d_{2} \tilde{\tilde{w}}, \quad u=d_{2} \tilde{u}, \quad d_{1}=d_{2} \tilde{d}_{1}
$$

These blow-ups resolve all codimension one singularities in the elliptically fibered CalabiYau manifold. However, there are still codimension two singularities of the elliptic fibration that have to be resolved. This has to be done via a small resolution in order to maintain a flat fibration. In the following we will consider one of the six possible small resolutions in [19, 20]. We perform the small resolutions at $\tilde{\tilde{w}}=\tilde{d}_{1}=0$ and at $\tilde{\tilde{w}}=d_{2}=0$ by introducing two new divisor sections $d_{3}, d_{4}$. Then, the resolution map reads

$$
\tilde{\tilde{w}}=d_{3} d_{4} \tilde{\tilde{w}}, \quad \tilde{d}_{1}=d_{4} \tilde{\tilde{d}}_{1}, \quad d_{2}=d_{3} \tilde{d}_{2}
$$

It can be checked explicitly that these final resolutions render the geometry smooth. Combining all the four resolution maps, we obtain the total variable transformations

$$
w \rightarrow \tilde{\tilde{d}}_{1} \tilde{\tilde{d}}_{2}^{2} d_{3}^{3} d_{4}^{2} \tilde{\tilde{w}}, \quad v \rightarrow \tilde{\tilde{d}}_{1} \tilde{d}_{2} d_{3} d_{4} \tilde{v}, \quad z_{2} \rightarrow \tilde{\tilde{d}}_{1} \tilde{d}_{2} d_{3} d_{4} \tilde{z}_{2}, \quad u \rightarrow \tilde{d}_{2} d_{3} \tilde{u}
$$

In order to shorten our notation we will in the following drop all the tildes of the variables on the resolution by abuse of notation. A consistent assignment of divisor classes on the resolved geometry is presented in (6.13), where we note that the coordinates displayed there are the coordinates on the resolution after dropping tildes.

Finally, we use the resolution map (6.21) to obtain the proper transform of the CalabiYau polynomial $p^{\prime}$ in (3.27). The fully resolved geometry $\hat{X}$ reads, after dropping superscripts ', as

$$
\begin{aligned}
p= & s_{6}\left(e_{1} e_{2}\right) u v w+s_{7}\left(d_{1} d_{4}\right) e_{2} v^{2} w+s_{8}\left(d_{2} d_{3}^{2} d_{4}\right) e_{1}^{2} u w^{2}+s_{9}\left(d_{1} d_{2} d_{3}^{2} d_{4}^{2}\right) e_{1} v w^{2} \\
& +z_{2} s_{5}\left(d_{2} d_{3}\right) e_{1}^{2} e_{2} u^{2} w+z_{2}^{2} s_{2}\left(d_{1} d_{2}\right) e_{1} e_{2}^{2} u^{2} v+z_{2}^{2} s_{3}\left(d_{1}^{2} d_{2} d_{4}\right) e_{2}^{2} u v^{2} \\
& +z_{2}^{3} s_{1}\left(d_{1} d_{2}^{2} d_{3}\right) e_{1}^{2} e_{2}^{2} u^{3} .
\end{aligned}
$$

We conclude with some remarks on codimension two singularities and the expected matter multiplets in the resolved geometry It is well-known, see e.g. [19] for a review, that 
the $\mathrm{SU}(5)$-singularity in the fiber enhances further at the codimension two loci $z_{2}=0$ with either $\beta_{5}=0$ or $P=0$. In our case, these loci were identified in (6.17). At the loci $\beta_{5}:=s_{6} \stackrel{!}{=} 0$ the fiber enhances to a $D_{5}$-type singularity which are the loci of 10-matter representations in F-theory. As we see from (6.17) the $\beta_{5}$ is generic. Thus there is only one 10-matter representation in an model with an additional $\mathrm{U}(1)^{2}$-sector at $\beta_{5}=0$. In contrast, the loci $P=0$, where the fiber enhances to an $I_{6}$-singularity and where 5 matter is located, split into five different curves described by $P_{i}=0, i=1, \ldots, 5$. Thus in an F-theory compactification with $\mathrm{SU}(5) \times \mathrm{U}(1)^{2}$ gauge symmetry there will be five different 5-matter representations with differing U(1)-charges. It would be interesting to investigate these geometries further and check anomaly cancellation in six dimensions by working out matter multiplicities along the lines of section 4.4 .

\section{Equivalence of $B l_{(1,0,0)} \mathbb{P}^{2}(1,2,3)$ and $B l_{(0,1,0)} \mathbb{P}^{2}(1,1,2)$ Elliptic Fibrations}

In this section we illustrate the power of birational transformations to show the equivalence of two different representations of an elliptic curve with one rational point. One representation is the $\mathrm{U}(1)$-restricted Tate model [39] in the blow-up $B l_{(1,0,0)} \mathbb{P}^{2}(1,2,3)$ and the other one is the hypersurface in $B l_{(0,1,0)} \mathbb{P}^{2}(1,1,2)$ studied in [43]. We show that elliptic fibrations constructed from these models agree via a birational map and are related to models with no rational section by extremal transitions. We strongly suspect that the logic presented in the following holds more generally and can also be applied to prove the birational equivalence with $d P_{1}$-model studied in [46] and equivalences of models with multiple sections. Our following discussion is based on the extremal transitions studied in 51$]^{18}$.

We start by motivating the equivalence of the U(1)-restricted Tate model, which can be realized as the toric hypersurface in $B l_{(1,0,0)} \mathbb{P}^{2}(1,2,3)$, with the toric hypersurface in $B l_{(0,1,0)} \mathbb{P}^{2}(1,1,2)$ by studying the two-dimensional toric ambient varieties.

\footnotetext{
${ }^{18}$ We also acknowledge very useful discussions with Antonella Grassi about the toric blow-up in $\mathbb{P}^{4}(1,1,1,3,6)$ and its effect on the toric Calabi-Yau hypersurface.
} 
The toric variety $B l_{(1,0,0)} \mathbb{P}^{2}(1,2,3)$ is represented by a polytope with vertices

\begin{tabular}{|rr||c|c||rr|r|}
\hline \multicolumn{2}{|c|}{ vertices } & & divisor class & $\ell^{(1)}$ & $\ell^{(2)}$ & $\tilde{\ell}^{(1)}$ \\
\hline-2 & -3 & $z$ & $H$ & 1 & 0 & 1 \\
1 & 0 & $x_{1}$ & $2 H-E_{1}$ & -1 & 1 & 0 \\
0 & 1 & $y_{1}$ & $3 H-E_{1}$ & 0 & 1 & 1 \\
1 & 1 & $e_{1}$ & $E_{1}$ & 3 & -1 & 2 \\
\hline
\end{tabular}

with only interior point the origin. We note that there are three points interior to the one-dimensional faces of the polytope. As before we presented the vertices as row-vectors in the first two columns, introduced the projective coordinates in the third column and displayed the toric divisor classes in the fourth column. $H$ denotes the proper transform of the hyperplane class on $\mathbb{P}^{2}(1,2,3)$ and $E_{1}$ is the exceptional divisor. The charge vectors $\ell^{(i)}$ in the fifth and sixth columns are the generators of the Mori cone, that describe the scaling relations among the coordinates. We note that the charge vector of $\mathbb{P}^{2}(1,2,3)$ is obtained as $\ell^{(1)}+3 \ell^{(2)}=(1,2,3,0)^{T}$. In terms of the coordinates in (7.1) the blow-down map $\pi$ to $\mathbb{P}^{2}(1,2,3)$ with coordinates $[x: y: z]$ reads

$$
\pi:\left[z: x_{1}: y_{1}: e_{1}\right] \mapsto[x: y: z]=\left[x_{1} e_{1}: y_{1} e_{1}: z\right]
$$

We also obtain the charge vector $\tilde{\ell}^{(1)}=\ell^{(1)}+\ell^{(2)}=(1,0,1,2)^{T}$, as denoted in the last column of $(7.1)$, with respect to which $\left[z: 1: y_{1}: e_{1}\right]$ scale as the homogeneous coordinates $[u: v: w]$ in $\mathbb{P}^{(2)}(1,1,2)$. In addition, we can make this manifest by forming the following $\mathbb{C}^{*}$-covariant combination of the coordinates $\left[z: x_{1}: y_{1}: e_{1}\right]$ as

$$
U \equiv \frac{z}{x_{1}}, \quad V \equiv \frac{y_{1}}{x_{1}}, \quad W \equiv e_{1}, \quad T \equiv x_{1}
$$

We readily check using (7.1) that these coordinates enjoy the $\mathbb{C}^{*}$-actions

\begin{tabular}{|l|rrrr|}
\hline & $U$ & $V$ & $W$ & $T$ \\
\hline \hline$\tilde{\ell}^{(1)}$ & 1 & 1 & 2 & 0 \\
$\tilde{\ell}^{(2)}$ & -1 & 0 & -1 & 1 \\
\hline
\end{tabular}

which are precisely the $\mathbb{C}^{*}$-actions defining $B l_{(0,1,0)} \mathbb{P}^{2}(1,1,2)$, cf. (7.6). Thus, we have a second blow-down map $\pi^{\prime}$ to $\mathbb{P}^{(2)}(1,1,2)$ resolving $[u: v: w]=[0: 1: 0]$ is obvious from (7.3) reading

$$
\pi^{\prime}:[U: V: W: T] \mapsto[u: v: w]=[U T: V: W T]
$$


In fact, by writing down all sections of the anti-canonical bundle of $B l_{(1,0,0)} \mathbb{P}^{2}(1,2,3)$ in the coordinates $(7.3)$ we precisely reproduce all sections of the canonical bundle of $B l_{(0,1,0)} \mathbb{P}^{(2)}(1,1,2)$. Thus, we see the birational equivalence of the two models. In the following we work out the birational maps in detail and recover the birational map, that was found in [43] algebraically, by a straightforward toric analysis and one toric blow-up.

Before proceeding we present the toric data of $B l_{(0,1,0)} \mathbb{P}^{(2)}(1,1,2)$. Its polytope reads

\begin{tabular}{|rr||c|c||rr|}
\hline \multicolumn{2}{|c|}{ vertices } & & divisor class & $\ell^{\prime(1)}$ & $-\tilde{\ell}^{(2)}$ \\
\hline 1 & 0 & $U$ & $H-E_{1}$ & -1 & 1 \\
-1 & -2 & $V$ & $H$ & 1 & 0 \\
0 & 1 & $W$ & $2 H-E_{1}$ & 0 & 1 \\
1 & 1 & $T$ & $E_{1}$ & 2 & -1 \\
\hline
\end{tabular}

where again the only interior point is the origin and there is one point interior to an one-dimensional face. The homogeneous coordinates in the third column scale under the relations displayed in the last two columns, that form a basis of the two-dimensional Moricone. We recover the scaling relation of $B l_{(0,1,0)} \mathbb{P}^{(2)}(1,1,2)$ in 7.4 as $\ell^{\prime(1)}=\tilde{\ell}^{(1)}+2 \tilde{\ell}^{(2)}$.

We perform our derivation of the birational equivalence of these two presentation of elliptic curves by first matching their respective singular Tate models and then by performing the toric resolution. We begin with recalling the U(1)-restricted Tate model. The Tate form (2.1) of the elliptic curve takes the form

$$
y^{2}+a_{1} x y z+a_{3} y z^{3}=x^{3}+a_{2} x^{2} z^{2}+a_{4} x z^{4}
$$

where we work in the affine patch with the coordinate of the exceptional divisor $e_{1}=1$. The coefficient $a_{6}$ has been removed torically, $a_{6} \equiv 0$, by adding the last point in (7.1) of the exceptional divisor to the polytope of $\mathbb{P}^{2}(1,2,3)$, which removes the point in the dual polytope to (7.1) corresponding to the monomial $a_{6} z^{6}$. Thus, an elliptically fibered Calabi-Yau manifold over a base $B$ with the global Tate model of the form (7.7) with generic coefficients $a_{i} \in \mathcal{O}\left(-i K_{B}\right)$ is readily constructed as the Calabi-Yau hypersurface or complete intersection in the ambient space obtained by fibering $B l_{(1,0,0)} \mathbb{P}^{2}(1,2,3)$ over the base $B$. In particular if $B$ admits a toric description, i.e. is either a toric variety or a toric complete intersection, we can construct this ambient space torically by fibering the polytope (7.1) over the polytope describing $B$. We refer to section 6 for the required structure of the polytope to construct such a toric fibration

Now we turn to the hypersurface in $B l_{(0,1,0)} \mathbb{P}^{(2)}(1,1,2)$. It has been shown in [43] 
that its Tate form reads

$$
y^{2}+\tilde{a}_{1} x^{\prime} y z+\tilde{a}_{3} y z^{3}=x^{\prime 3}+\tilde{a}_{2} x^{\prime 2} z^{2}+\tilde{a}_{4} x^{\prime} z^{4}+\tilde{a}_{6} z^{6}
$$

with the Tate coefficients $\tilde{a}_{i}$ given by

$$
\tilde{a}_{1}=\frac{2 c_{3}}{b}, \quad \tilde{a}_{2}=\frac{b^{2} c_{2}-c_{3}^{2}}{b^{2}}, \quad \tilde{a}_{3}=b c_{1}, \quad \tilde{a}_{4}=-b^{2} c_{0}, \quad \tilde{a}_{6}=\tilde{a}_{2} \tilde{a}_{4}=-b^{2} c_{0}\left(b^{2} c_{2}-c_{3}^{2}\right) .
$$

We note immediately that these coefficients are not generic, namely we have five different coefficients parametrized by only four independent variables $\tilde{a}_{1}, \tilde{a}_{2}, \tilde{a}_{3}$ and $\tilde{a}_{4}$. Indeed we can eliminate this redundancy by a simple variable transformation,

$$
x^{\prime}=x-\tilde{a}_{2} z^{2}
$$

which is a subgroup of the automorphisms of $\mathbb{P}^{2}(1,2,3)$. The Tate coefficients $a_{i}^{\prime}$ with respect to the new coordinates $\left[x^{\prime}: y: z\right]$ read

$$
a_{1}^{\prime}=\tilde{a}_{1}, \quad a_{2}^{\prime}=-2 \tilde{a}_{2}, \quad a_{3}^{\prime}=\tilde{a}_{3}-\tilde{a}_{1} \tilde{a}_{2}, \quad a_{4}^{\prime}=\tilde{a}_{2}^{2}+\tilde{a}_{4}, \quad a_{6}^{\prime}=0 .
$$

When considering an elliptic fibration, these coefficients are identified with generic sections of appropriate line bundles on the base $B$ of the fibration. We then identify $a_{i}^{\prime} \equiv a_{i}$ in the U(1)-restricted Tate model (7.7). Thus we have shown by matching the Tate forms (7.7) and (7.8) that the elliptic curves in $B l_{(1,0,0)} \mathbb{P}^{2}(1,2,3)$, i.e. the $\mathrm{U}(1)$-restricted Tate model, and in $B l_{(0,1,0)} \mathbb{P}^{2}(1,1,2)$ are birationally equivalent.

We emphasize that this equivalence does not hold for general elliptically fibered Calabi-Yau manifolds. We claim that the equivalence only holds if the Calabi-Yau constraint with general fiber in $B l_{(0,1,0)} \mathbb{P}^{2}(1,1,2)$ takes globally the form

$$
w^{2}+b w v^{2}=u\left(c_{0} u^{3}+c_{1} u^{2} v+c_{2} u v^{2}+c_{3} v^{3}\right)
$$

where it is crucial that the coefficient of $w^{2}$ is one, which puts certain restrictions on the construction of the elliptic fibration. Fibrations of these type have been studied in [43] for the example of $B=\mathbb{P}^{2}$. In this case the coefficients were identified as

$$
b \equiv b_{n}, \quad c_{3}=f_{3+n}, \quad c_{2}=3 f_{6}, \quad c_{1}=2 f_{9-n}, \quad c_{0}=f_{12-2 n} .
$$

where the integers in the subscript denote the degree of the polynomial on $\mathbb{P}^{2}$. We see with these identifications that the Tate coefficients (7.11) are indeed general polynomials 
and the fibration becomes equivalent to a generic U(1)-restricted Tate model (7.7) as claimed.

After having proved this equivalence of the two elliptic fibrations with Tate models of the form (7.7) and (7.8) let us rederive the birational transformation between (7.7) in $B l_{(1,0,0)} \mathbb{P}^{2}(1,2,3)$ and the elliptic curve 7.12 in $B l_{(0,1,0)} \mathbb{P}^{2}(1,1,2)$. As we will demonstrate momentarily this will involve the single toric blow-up $B l_{(1,0,0)} \mathbb{P}^{2}(1,2,3) \rightarrow$ $\mathbb{P}^{2}(1,2,3)$ at $x=y=0$, the identification of coordinates of coordinates $(7.3)$ and the action of a simple automorphism bringing the constraint into the form (7.6).

First we note that the Tate model (7.7) is singular at codimension two in the base when $a_{3}=a_{4}=0$, or equivalently in the parametrization (7.11) when

$$
\frac{1}{2} b c_{1}-c_{2} \frac{c_{3}}{b}+\frac{c_{3}^{3}}{b^{3}}=\left(\frac{c_{3}^{2}}{b^{2}}-c_{2}\right)^{2}-b^{2} c_{0}=0
$$

All these singular loci in the base are contained in the intersection of the surface $x=y=0$ with the elliptic curve (7.8). Thus all these singularities are resolved by a single toric blow-up along at $x=y=0$. We perform the toric blow-up in (7.7) by setting

$$
x=x_{1} e_{1}=W T, \quad y=y_{1} e_{1}=V W T, \quad z=U T
$$

where we have also used the coordinates $(7.3)$ and have at the same time performed the coordinate transformation (7.10) to eliminate $\tilde{a}_{6}$ in (7.8). Inserting this into (7.7) we obtain

$$
\begin{aligned}
& W^{2} T-V^{2} W-2 \frac{c_{3}}{b} U V W T+2\left(\frac{c_{3}^{2}}{b^{2}}-c_{2}\right) U^{2} W T^{2} \\
= & U\left(\left(b^{2} c_{0}-c_{2}^{2}+2 c_{2} \frac{c_{3}^{2}}{b^{2}}-\frac{c_{3}^{4}}{b^{4}}\right)(U T)^{3}+\left(b c_{1}-2 c_{2} \frac{c_{3}}{b}+2 \frac{c_{3}^{3}}{b^{3}}\right) V(U T)^{2}\right),
\end{aligned}
$$

where we have canceled a factor $T^{2} W$. We readily observe that this constraint is mapped under the blow-down map (7.5) to the quartic in $\mathbb{P}^{2}(1,1,2)$ as claimed. Then we perform the simple variable transformation

$$
W=W^{\prime}+\frac{c_{3}}{b} U V+\left(c_{2}-\frac{c_{3}^{2}}{b^{2}}\right) T U^{2}, \quad T=T^{\prime} / b
$$

in order to bring 7.16 into the form 7.12 ,

$$
W^{2} T-b V^{2} W=U\left(c_{0} U^{3} T^{3}+c_{1} V U^{2} T^{2}+c_{2} V^{2} U T+c_{3} V^{3}\right),
$$


where we multiplied by $b$ and, by abuse of notation, denoted the new coordinates again by $W$ and $T$. In these coordinates 7.15 reads

$$
\begin{aligned}
x & =\frac{1}{b^{2}} T\left(b W+c_{3} U V+\left(c_{2}-\frac{c_{3}^{2}}{b^{2}}\right) T U^{2}\right), \\
y & =\frac{1}{b^{2}} V T\left(b W+c_{3} U V+\left(c_{2}-\frac{c_{3}^{2}}{b^{2}}\right) T U^{2}\right) \\
z & =\frac{1}{b} T U
\end{aligned}
$$

where we again denote the shifted coordinates 7.17 by $W$ and $T$.

Finally, we relate the coordinates $\tilde{x}, \tilde{y}$ defined in $(2.3)$ that bring the $\mathrm{U}(1)$-restricted Tate model (7.7) into Weierstrass form to the coordinates of $[U: V: W: T]$ of $B l_{(0,1,0)} \mathbb{P}^{2}(1,1,2)$. This amounts to simply writing out the transformation $(2.3)$ in terms of $x$ and $y$ given by (7.19). First, we evaluate using the Tate form (7.7) the parameters

$$
b_{2}=12\left(\frac{c_{3}^{2}}{b^{2}}-\frac{2}{3} c_{2}\right), \quad a_{1}=2 \frac{c_{3}}{b}, \quad a_{3}=2\left(\frac{1}{2} b c_{1}-c_{2} \frac{c_{3}}{b}+\frac{c_{3}^{3}}{b^{3}}\right)
$$

Then we readily plug this into 2.3 with 7.19 to obtain

$$
\begin{aligned}
\tilde{x} & =\frac{1}{b^{2}} T\left(c_{3} U V+\frac{1}{3} c_{2} T U^{2}+b W\right), \\
\tilde{y} & =\frac{1}{b^{3}} T\left(b c_{3} U V^{2}+b c_{2} T U^{2} V+\frac{1}{2} b c_{1} T^{2} U^{3}+c_{3} T U W+b^{2} V W\right), \\
z & =\frac{1}{b} T U
\end{aligned}
$$

where, as before, $W$ and $T$ are to be understood as the shifted coordinates in $(7.17)$. We note that we can use the $\mathbb{C}^{*}$-action on $[z: \tilde{x}: \tilde{y}]$ to cancel the prefactors $\frac{1}{b^{k}}, k=1,2,3$.

We readily recognize the birational transformation ${ }^{19}(7.21)$ as the birational map worked out in [43] to map the Weierstrass model of the elliptic fibration under consideration to the quartic 7.12 . In fact, by using the $\mathbb{C}^{*}$-action we can eliminate the prefactor of $T$ in 7.21 in front of $z$. Then we identify the coordinates of $\mathbb{P}^{2}(1,1,2)$ in the conventions of [43] as

$$
u:=U, \quad v:=\frac{V}{T}, \quad w:=\frac{W}{T} .
$$

\footnotetext{
${ }^{19}$ Its inverse is readily constructed by simply solving 7.21 for $U, V, W$.
} 
Thus, we have found the same birational map from a simple toric resolution $(7.15)$ and identification of charge vectors. Although both maps (7.15) and (7.21) are blow-ups at $x=y=0$ respectively $\tilde{x}=\tilde{y}=0$, the former map is considerably more natural.

\section{Outlook}

In this work we have analyzed F-theory vacua with $\mathrm{U}(1) \times \mathrm{U}(1)$ gauge group from compactifications on elliptically fibered Calabi-Yau manifolds with rank two Mordell-Weil group. We have derived from first principles that one has to depart from the Tate and Weierstrass form to represent an elliptic curve $\mathcal{E}$ with two rational points and its resolved elliptic fibrations: the appropriate presentation is the toric Calabi-Yau one-fold in $d P_{2}$. We have found the birational maps to its Tate and Weierstrass model and the coordinates of its two rational points in Weierstrass form. In addition we have explicitly studied its resolved elliptic fibrations $\hat{X}$ over a base $B$, that we have classified for $B=\mathbb{P}^{2}$. We have seen explicitly that one has to give up the paradigm of a holomorphic zero section in F-theory. This has also been confirmed by analyzing the codimension two singularities of the fibration $\hat{X}$, that determine the F-theory matter spectrum. The spectrum that we work out in detail and in generality is only consistent with six-dimensional anomaly cancellation if the zero section wraps fiber components over codimension two loci.

We have also constructed explicitly resolved toric Calabi-Yau threefolds with MordellWeil group of rank two for concrete six-dimensional F-theory compactifications with $\mathrm{U}(1) \times \mathrm{U}(1)$-sectors. We have two examples with only $\mathrm{U}(1) \times \mathrm{U}(1)$-gauge group, that differ in the complexity of their rational sections and matter sector. In both cases we determine the full anomaly-free matter spectrum. In a third example we add the nonAbelian sector of an SU(5)-gauge theory, i.e. construct an F-theory compactification with $\mathrm{SU}(5) \times \mathrm{U}(1) \times \mathrm{U}(1)$ gauge group. We explicitly write down the corresponding fourdimensional polytope. We observe a splitting of the 5 -matter curve into five different components, in other words matter in the 5 -representation splits into five matter representation $\mathbf{5}_{\left(q_{1}, q_{2}\right)}$ differing by their $\mathrm{U}(1)^{2}$-charges. The $\mathbf{1 0}$ matter curves does not split. We conclude by an analysis of extremal transitions and birational equivalences of elliptic fibrations with different fiber types. We employ these techniques to relate the U(1)-

restricted Tate model with fiber in $B l_{(1,0,0)} \mathbb{P}^{2}(1,2,3)$ to the fibration with general elliptic fiber in $B l_{(0,1,0)} \mathbb{P}^{2}(1,1,2)$.

It would be interesting to explore the 'landscape' of elliptic fibrations with different 
fiber types in F-theory. A possible starting point to study such different fibrations is to construct and analyze elliptic fibrations with general elliptic fiber realized as a CalabiYau onefold in all the different toric varieties corresponding to the 16 two-dimensional reflexive polytopes. This might also be relevant for a partial classification of Abelian sectors in F-theory. Furthermore, the systematic inclusion of non-Abelian sectors as well as the study of their matter spectrum and anomaly cancellation along the lines of sections 4 and 5 would be desirable. In particular, the general check of the gravitational anomaly, done here only for examples, can be used to prove the completeness of the study of codimension two singularities. Mathematically this translates into the Euler number of the resolution $\hat{X}$ [62]. We also refer to the early study of [63] on the connection between the rank of the Mordell-Weil group and the Euler number for elliptic fibrations of different fiber types. Finally and of immediate importance for the full understanding of four-dimensional F-theory vacua is the systematic extension of our techniques to CalabiYau fourfolds, codimension three phenomena and the structure of $G_{4}$-fluxes. We will return to the above issues in a future work [64].

\section{Acknowledgments}

We gratefully acknowledge discussions and correspondence with Lasha Berezhiani,Yi-Zen Chu, Thomas Grimm, Albrecht Klemm, Daniel Park and Maximilian Poretschkin. We thank especially Antonella Grassi for many useful discussions and comments. DK thanks the Bethe Center for Theoretical Physics Bonn for hospitality. This work is supported in part by DOE grant DE-SC0007901, the Fay R. and Eugene L. Langberg Endowed Chair and the Slovenian Research Agency (ARRS).

\section{A More Details on the Elliptic Curve in $d P_{2}$}

In this appendix we present the results of the explicit calculation of the Tate and Weierstrass model for the elliptic curve (3.4) in $d P_{2}$ that we omitted in the main text in section 3.1 .

First we summarize the coefficients $a_{i}$ of the Tate form

$$
y^{2}-x^{3}+a_{1} x y z-a_{2} x^{2} z^{2}+a_{3} y z^{3}-a_{4} x z^{4}-a_{6} z^{6}=0 .
$$


Using the results 3.18 and 3.20 for the sections $x$ and $y$ in Tate form we obtain

$$
\begin{aligned}
a_{1}= & \frac{1}{s_{7} s_{8}-s_{6} s_{9}}\left(s_{6} s_{7} s_{8}-s_{6}^{2} s_{9}+2 s_{5} s_{7} s_{9}+2 s_{3} s_{8} s_{9}-2 s_{2} s_{9}^{2}\right), \\
a_{2}= & -\frac{1}{\left(s_{7} s_{8}-s_{6} s_{9}\right)^{2}}\left(s_{5} s_{7}^{3} s_{8}^{2}+s_{3} s_{7}^{2} s_{8}^{3}-s_{5} s_{6} s_{7}^{2} s_{8} s_{9}-s_{3} s_{6} s_{7} s_{8}^{2} s_{9}-2 s_{2} s_{7}^{2} s_{8}^{2} s_{9}\right. \\
& +s_{5}^{2} s_{7}^{2} s_{9}^{2}+2 s_{3} s_{5} s_{7} s_{8} s_{9}^{2}+3 s_{2} s_{6} s_{7} s_{8} s_{9}^{2}+s_{3}^{2} s_{8}^{2} s_{9}^{2}-s_{2} s_{6}^{2} s_{9}^{3}-2 s_{2} s_{5} s_{7} s_{9}^{3} \\
& \left.-2 s_{2} s_{3} s_{8} s_{9}^{3}+s_{2}^{2} s_{9}^{4}\right), \\
a_{3}= & -s_{2} s_{7} s_{8}-s_{3} s_{5} s_{9}+s_{2} s_{6} s_{9}-s_{1} s_{7} s_{9}, \\
a_{4}= & \frac{1}{s_{7} s_{8}-s_{6} s_{9}}\left(s_{3} s_{5} s_{7}^{2} s_{8}^{2}+s_{1} s_{7}^{3} s_{8}^{2}-s_{3} s_{5} s_{6} s_{7} s_{8} s_{9}-s_{1} s_{6} s_{7}^{2} s_{8} s_{9}+s_{3} s_{5}^{2} s_{7} s_{9}^{2}\right. \\
& \left.+s_{1} s_{5} s_{7}^{2} s_{9}^{2}+s_{3}^{2} s_{5} s_{8} s_{9}^{2}+2 s_{1} s_{3} s_{7} s_{8} s_{9}^{2}-s_{2} s_{3} s_{5} s_{9}^{3}-s_{1} s_{3} s_{6} s_{9}^{3}-s_{1} s_{2} s_{7} s_{9}^{3}\right), \\
a_{6}= & s_{1} s_{3}\left(-s_{7}^{2} s_{8}^{2}+s_{6} s_{7} s_{8} s_{9}-s_{5} s_{7} s_{9}^{2}-s_{3} s_{8} s_{9}^{2}+s_{2} s_{9}^{3}\right) .
\end{aligned}
$$

From this we readily obtain the polynomials

$$
\begin{aligned}
b_{2}= & s_{6}^{2}-4 s_{5} s_{7}-4 s_{3} s_{8}+8 s_{2} s_{9}, \\
b_{4}= & 2 s_{2}^{2} s_{9}^{2}+s_{1} s_{7}\left(2 s_{7} s_{8}-s_{6} s_{9}\right)+s_{2}\left(s_{6}^{2} s_{9}-s_{6} s_{7} s_{8}-2 s_{5} s_{7} s_{9}\right) \\
& +s_{3}\left(2 s_{5} s_{7} s_{8}-s_{5} s_{6} s_{9}-2 s_{2} s_{8} s_{9}+2 s_{1} s_{9}^{2}\right), \\
b_{6}= & \left(s_{2} s_{7} s_{8}+s_{3} s_{5} s_{9}-s_{2} s_{6} s_{9}+s_{1} s_{7} s_{9}\right)^{2} \\
& -4 s_{1} s_{3}\left(s_{7}^{2} s_{8}^{2}+s_{9}^{2}\left(s_{3} s_{8}-s_{2} s_{9}\right)+s_{7} s_{9}\left(s_{5} s_{9}-s_{6} s_{8}\right)\right),
\end{aligned}
$$

in terms of which we calculate the polynomials $f, g$ for the Weierstrass model according 
to $(2.4)$ as

$$
\begin{aligned}
f & =\frac{1}{48}\left[-s_{6}^{4}+8 s_{6}^{2}\left(s_{5} s_{7}+s_{3} s_{8}+s_{2} s_{9}\right)-24 s_{6}\left(s_{2} s_{7} s_{8}+s_{3} s_{5} s_{9}+s_{1} s_{7} s_{9}\right)\right. \\
& \left.+16\left(-s_{5}^{2} s_{7}^{2}+3 s_{1} s_{7}^{2} s_{8}-s_{3}^{2} s_{8}^{2}+s_{2} s_{3} s_{8} s_{9}-s_{2}^{2} s_{9}^{2}+3 s_{1} s_{3} s_{9}^{2}+s_{5} s_{7}\left(s_{3} s_{8}+s_{2} s_{9}\right)\right)\right] \\
g & =\frac{1}{864}\left[s_{6}^{6}-12 s_{6}^{4}\left(s_{5} s_{7}+s_{3} s_{8}+s_{2} s_{9}\right)+36 s_{6}^{3}\left(s_{2} s_{7} s_{8}+s_{3} s_{5} s_{9}+s_{1} s_{7} s_{9}\right)\right. \\
& +24 s_{6}^{2}\left(2 s_{5}^{2} s_{7}^{2}+2 s_{3}^{2} s_{8}^{2}+s_{2} s_{3} s_{8} s_{9}+2 s_{2}^{2} s_{9}^{2}+s_{5} s_{7}\left(s_{3} s_{8}+s_{2} s_{9}\right)-3 s_{1}\left(s_{7}^{2} s_{8}+s_{3} s_{9}^{2}\right)\right) \\
& +8\left(-8 s_{5}^{3} s_{7}^{3}-72 s_{1} s_{3} s_{7}^{2} s_{8}^{2}-8 s_{3}^{3} s_{8}^{3}+27 s_{1}^{2} s_{7}^{2} s_{9}^{2}-72 s_{1} s_{3}^{2} s_{8} s_{9}^{2}-8 s_{2}^{3} s_{9}^{3}+3 s_{2}^{2} s_{8}\left(9 s_{7}^{2} s_{8}+4 s_{3} s_{9}^{2}\right)\right. \\
& +6 s_{5} s_{7}\left(6 s_{1} s_{7}^{2} s_{8}+2 s_{3}^{2} s_{8}^{2}+s_{2} s_{3} s_{8} s_{9}+2 s_{2}^{2} s_{9}^{2}-3 s_{1} s_{3} s_{9}^{2}\right)+6 s_{2} s_{9}\left(-3 s_{1} s_{7}^{2} s_{8}+2 s_{3}^{2} s_{8}^{2}+6 s_{1} s_{3} s_{9}^{2}\right) \\
& \left.+3 s_{5}^{2}\left(4 s_{3} s_{7}^{2} s_{8}+4 s_{2} s_{7}^{2} s_{9}+9 s_{3}^{2} s_{9}^{2}\right)\right)-144 s_{6}\left(s_{2}^{2} s_{7} s_{8} s_{9}+\left(s_{1} s_{5} s_{7}^{2}+s_{3}^{2} s_{5} s_{8}+s_{3} s_{7}\left(s_{5}^{2}-5 s_{1} s_{8}\right)\right) s_{9}\right. \\
& \left.\left.+s_{2}\left(s_{5} s_{7}^{2} s_{8}+s_{3} s_{7} s_{8}^{2}+s_{3} s_{5} s_{9}^{2}+s_{1} s_{7} s_{9}^{2}\right)\right)\right] .
\end{aligned}
$$

With these results the discriminant is straightforward and can be provided upon request.

\section{B Nagell's Algorithm: Cubic to Weierstrass}

Any Calabi-Yau hypersurfaces in a two-dimensional toric variety is an elliptic curve $\mathcal{E}$. In the following we will find the birational map of the cubic in $\mathbb{P}^{2}$ to Weierstrass form. In contrast to the method used in the main text in section 3.1 we employ Nagell's algorithm 20 , which is an alternative way to obtain the discriminant of $\mathcal{E}$.

Before delving into the details of this algorithm, let us summarize the general idea on which it is based. Every elliptic curve $\mathcal{E}$ can be described as a double cover of an $\mathbb{P}^{1}$ with all information about the curve $\mathcal{E}$ encoded in the four branch points and the two branch cuts in-between. Such a double cover is generically described as

$$
\xi^{2}=q_{4}(\tau)=\left(t-t_{1}\right)\left(t-t_{2}\right)\left(t-t_{3}\right)\left(t-t_{4}\right)
$$

where $t$ is the coordinate on $\mathbb{P}^{1}$ and the $\tau_{i}$ are the branch points. By the action of $\operatorname{SL}(2, \mathbb{C})$ we can always move these points to 0,1 and $\infty$ with only one movable branch point that specifies the complex structure of $\mathcal{E}$. If we move only one of the branch points to infinity, we recover upon identifying $\xi \equiv y$ and $t \equiv x$ the cubic polynomial on the right hand side of the Weierstrass form (2.2) in the affine patch $z$. Thus, finding the Weierstrass model

\footnotetext{
${ }^{20}$ We note that the following results have been worked out independently by Maximilian Poretschkin.
} 
in all of the above cases reduces to finding an appropriate map to a $\mathbb{P}^{1}$ such that the Calabi-Yau equation takes the form of a double cover over this $\mathbb{P}^{1}$. Upon mapping one branch point in $t$ to infinity one immediately recovers the Weierstrass model.

The application of this idea to the cubic curve is the foundation of Nagell's algorithm that we present in the following. Consider a cubic curve in $\mathbb{P}^{2}$ with projective coordinates $[u: v: w]$ of the form

$$
p=s_{1} u^{3}+s_{2} u^{2} v+s_{3} u v^{2}+s_{4} v^{3}+s_{5} u^{2} w+s_{6} u v w+s_{7} v^{2} w+s_{8} u w^{2}+s_{9} v w^{2}+s_{10} w^{3} .
$$

For the moment we assume that the coefficients $s_{i}$ are in a field $K$, e.g. $K=\mathbb{C}$. Then, for generic $s_{i}$ the cubic $(\overline{B .2})$ defines a smooth elliptic curve $\mathcal{E}$. In applications to elliptic fibrations $\pi: X \rightarrow B$ of $\mathcal{E}$ over a base $B$, the $s_{i}$ are sections of a line bundle $\mathcal{L}^{i}$ and are locally represented by polynomials in local coordinates on the base $B$.

The cubic (B.2) can be brought into Weierstrass form by application of Nagell's algorithm, where we follow the exposition of [65]. In the case at hand, the above mentioned map to an appropriate $\mathbb{P}^{1}$, which turns the cubic $(B .2)$ into a double cover of the form (B.1), is given by mapping to the slopes $t$ of lines through a given point $Q$ on $\mathcal{E}$. Concretely, we first assume that $s_{10}=0$ which can be achieved by a coordinate transformation ${ }^{21}$ of $u$ and $v$. Then the point $P=[0,0,1]$ is on the curve (B.2). Next we assume that $s_{9} \neq 0$ without loss of generality since we can always interchange $u$ and $v$ and both $s_{8}=s_{9}=0$ implies that the corresponding elliptic curve $\mathcal{E}$ is singular at $P$.

Then we define

$$
p=F_{3}(u, v)+F_{2}(u, v) w+F_{1}(u, v) w^{2},
$$

i.e. $F_{i}(u, v)$ is the coefficient polynomial of the term $w^{3-i}$ in $(\bar{B} .2)$. Every line in $\mathbb{P}^{2}$ meets the curve $(\overline{B .2})$ in three points. The tangent at $P$ is the line meeting $\mathcal{E}$ twice at $P$. The point $P$ becomes a double point of $(\bar{B} .2)$ precisely when $F_{1}=0$ along the line $v=t_{0} u$, thus, the tangent at $P$ is described by the equation

$$
F_{1}=s_{8} u+s_{9} v=0 .
$$

In other words, the slope $t_{0}$ of the tangent at $P$ is $t_{0}=-\frac{s_{8}}{s_{9}}$. The tangent meets $p$ in another point $Q$, see figure 6 for an illustration of this situation. Its coordinates are $Q=\left[-e_{2} s_{9}: e_{2} s_{8}: e_{3}\right]$ where we defined $e_{i}=F_{i}\left(s_{9},-s_{8}\right)^{22}$. If $e_{2}=0$, then $P=Q$

\footnotetext{
${ }^{21}$ In general, however, this coordinate transformation involves the third roots of the coefficients $b_{i}$ which might not be in the field $K$ under consideration.

${ }^{22}$ Note that the $e_{i}$ here should not be confused with the coordinates $e_{1}, e_{2}$ of the exceptional divisors in $d P_{2}$ in the main text.
} 


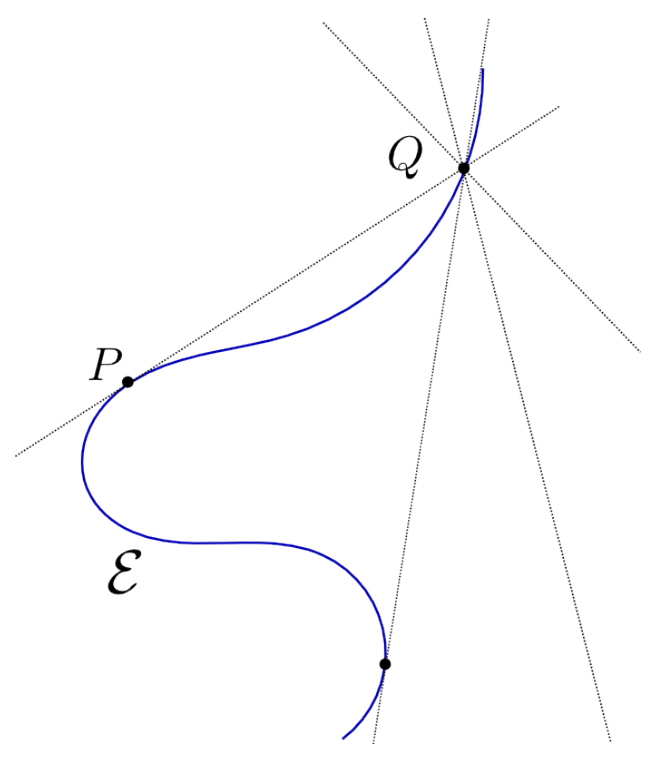

Figure 6: Cubic curve $\mathcal{E}$ (in blue) with the two points $P$ and $Q$. The tangent at $P$ intersects $\mathcal{E}$ at the point $Q$. This implies that the slope $t_{0}$ of the tangent is a root of the discriminant $\delta(t)$ in (B.8), since $\delta(t)=0$ for double points of $\mathcal{E}$ along lines through $Q$.

is a triple point or flex of $\mathcal{E}$ and if $e_{3}=0$, then $Q=\left[-s_{9}, s_{8}, 0\right]$ is at infinity. Both $e_{2}=e_{3}=0$ implies that $p=0$ along the line $F_{1}=0$ or $p=F_{1}(u, v) q_{2}(u, v, w)$ for a quadratic polynomial in $[u: v: w]$. This means that the curve $\mathcal{E}$ is reducible with components $\mathbb{P}^{1}$ and the quadric in $\mathbb{P}^{2}$, thus, not elliptic.

Next we perform a variable transformation that centers $Q$ at $u^{\prime}=v^{\prime}=0$. The variable change reads

$$
\begin{aligned}
& e_{3} \neq 0: \quad u=u^{\prime}-s_{9} \frac{e_{2}}{e_{3}} w, \quad v=v^{\prime}+s_{8} \frac{e_{2}}{e_{3}} w, \\
& e_{3}=0: \quad u=u^{\prime}-s_{9} w, \quad v=v^{\prime}+s_{8} w,
\end{aligned}
$$

where we have to distinguish again the cases $e_{3} \neq 0$ and $e_{3}=0$. We denote the constraint $p$ in the new coordinates as

$$
p^{\prime}=f_{3}\left(u^{\prime}, v^{\prime}\right)+f_{2}\left(u^{\prime}, v^{\prime}\right) w+f_{1}\left(u^{\prime}, v^{\prime}\right) w^{2}
$$

where $f_{i}$ denote homogeneous polynomials of degree $i$ in $\left(u^{\prime}, v^{\prime}\right)$. Now we consider the lines $v^{\prime}=t u^{\prime}$ through $Q$. Their intersections with $\mathcal{E}$ in generically two other points are 
determined by the intersection with the constraint $p^{\prime}$ reading

$$
u^{\prime}\left(\phi_{3}(t) u^{\prime 2}+\phi_{2}(t) u^{\prime} w+\phi_{1}(t) w^{2}\right)=0 .
$$

Here we readily cancel the factor $u^{\prime}=0$ which is the point $Q$ itself. Furthermore, we have defined $\phi_{i}(t)=f_{i}(1, t)$. We determine the two roots of this quadratic equation as a function of the slope $t$ by multiplying $(\overline{B .7})$ with $4 \phi_{3}(t)$. Then we complete the square yielding

$$
\left(2 \phi_{3}(t) u^{\prime}+\phi_{2}(t) w\right)^{2}=\delta(t) w^{2}, \quad \delta(t)=\phi_{2}^{2}(t)-4 \phi_{1}(t) \phi_{3}(t)
$$

where in the case at hand the $\phi_{i}$ are lengthy polynomials in $t$ that we omit. The double roots are the zeros $t_{i}$ of the discriminant $\delta(t)$ of the quadratic equation in $u^{\prime}$, of which there are generically four as $\delta(t)$ is a fourth order polynomial in $t$. Geometrically, the lines with the slopes $t_{i}$ are those lines through $Q$, that are a tangent or in other words are a double point of $\mathcal{E}$ at a different point. By construction, we already know one such line that is the tangent at $P$ given by (B.4) with slope $t_{0}=-\frac{s_{8}}{s_{9}}$, cf. figure 6

In other words we know the linear factor $\left(t-t_{0}\right)$ of $\delta(t)$. Thus we can perform the variable transformation $t=t_{0}+\frac{1}{\tau}$ to move this root to infinity $\tau=\infty$. Consequently we obtain a third order polynomial

$$
\rho(\tau)=\tau^{4} \delta\left(t_{0}+\frac{1}{\tau}\right)=c \tau^{3}+d \tau^{2}+e \tau+k,
$$

for coefficients $c, d, e$ and $k$, that in the case at hand read

$$
\begin{aligned}
& c=4\left(s_{2} s_{8}+\frac{s_{4} 3_{8}^{3}}{s_{9}^{2}}-\frac{s_{3} s_{8}^{2}}{s_{9}}-s_{1} s_{9}\right), \quad d=s_{6}^{2}-4 s_{5} s_{7}+8 s_{3} s_{8}-\frac{12 s_{4} s_{8}^{2}}{s_{9}}-4 s_{2} s_{9}, \\
& e=\frac{2}{s_{4} s_{8}^{3}-s_{3} s_{8}^{2} s_{9}+s_{2} s_{8} s_{9}^{2}-s_{1} s_{9}^{3}}\left(6 s_{4}^{2} s_{8}^{4}-s_{4} s_{6}^{2} s_{8}^{2} s_{9}+4 s_{4} s_{5} s_{7} s_{8}^{2} s_{9}-8 s_{3} s_{4} s_{8}^{3} s_{9}-s_{4} s_{5} s_{6} s_{8} s_{9}^{2}\right. \\
& +s_{3} s_{6}^{2} s_{8} s_{9}^{2}-2 s_{3} s_{5} s_{7} s_{8} s_{9}^{2}-s_{2} s_{6} s_{7} s_{8} s_{9}^{2}+2 s_{1} s_{7}^{2} s_{8} s_{9}^{2}+2 s_{3}^{2} s_{8}^{2} s_{9}^{2}+6 s_{2} s_{4} s_{8}^{2} s_{9}^{2}+2 s_{4} s_{5}^{2} s_{9}^{3}-s_{3} s_{5} s_{6} s_{9}^{3} \\
& \left.+2 s_{2} s_{5} s_{7} s_{9}^{3}-s_{1} s_{6} s_{7} s_{9}^{3}-2 s_{2} s_{3} s_{8} s_{9}^{3}-6 s_{1} s_{4} s_{8} s_{9}^{3}+2 s_{1} s_{3} s_{9}^{4}\right), \\
& k=\frac{-s_{9}}{\left(s_{4} s_{8}^{3}-s_{3} s_{8}^{2} s_{9}+s_{2} s_{8} s_{9}^{2}-s_{1} s_{9}^{3}\right)^{2}}\left(4 s_{4}^{3} s_{8}^{6}-s_{4}^{2} s_{6}^{2} s_{8}^{4} s_{9}+4 s_{4}^{2} s_{5} s_{7} s_{8}^{4} s_{9}-8 s_{3} s_{4}^{2} s_{8}^{5} s_{9}-2 s_{4}^{2} s_{5} s_{6} s_{8}^{3} s_{9}^{2}\right. \\
& +2 s_{3} s_{4} s_{6}^{2} s_{8}^{3} s_{9}^{2}-4 s_{3} s_{4} s_{5} s_{7} s_{8}^{3} s_{9}^{2}-2 s_{2} s_{4} s_{6} s_{7} s_{8}^{3} s_{9}^{2}+4 s_{1} s_{4} s_{7}^{2} s_{8}^{3} s_{9}^{2}+4 s_{3}^{2} s_{4} s_{8}^{4} s_{9}^{2}+8 s_{2} s_{4}^{2} s_{8}^{4} s_{9}^{2} \\
& +3 s_{4}^{2} s_{5}^{2} s_{8}^{2} s_{9}^{3}-s_{3}^{2} s_{6}^{2} s_{8}^{2} s_{9}^{3}+6 s_{2} s_{4} s_{5} s_{7} s_{8}^{2} s_{9}^{3}+2 s_{2} s_{3} s_{6} s_{7} s_{8}^{2} s_{9}^{3}-6 s_{1} s_{4} s_{6} s_{7} s_{8}^{2} s_{9}^{3}-s_{2}^{2} s_{7}^{2} s_{8}^{2} s_{9}^{3} \\
& -8 s_{2} s_{3} s_{4} s_{8}^{3} s_{9}^{3}-8 s_{1} s_{4}^{2} s_{8}^{3} s_{9}^{3}-2 s_{3} s_{4} s_{5}^{2} s_{8} s_{9}^{4}+2 s_{3}^{2} s_{5} s_{6} s_{8} s_{9}^{4}-4 s_{2} s_{4} s_{5} s_{6} s_{8} s_{9}^{4}+4 s_{1} s_{4} s_{6}^{2} s_{8} s_{9}^{4} \\
& -2 s_{2} s_{3} s_{5} s_{7} s_{8} s_{9}^{4}+2 s_{1} s_{4} s_{5} s_{7} s_{8} s_{9}^{4}-2 s_{1} s_{3} s_{6} s_{7} s_{8} s_{9}^{4}+2 s_{1} s_{2} s_{7}^{2} s_{8} s_{9}^{4}+4 s_{2}^{2} s_{4} s_{8}^{2} s_{9}^{4}+8 s_{1} s_{3} s_{4} s_{8}^{2} s_{9}^{4} \\
& \left.-s_{3}^{2} s_{5}^{2} s_{9}^{5}+4 s_{2} s_{4} s_{5}^{2} s_{9}^{5}-4 s_{1} s_{4} s_{5} s_{6} s_{9}^{5}+2 s_{1} s_{3} s_{5} s_{7} s_{9}^{5}-s_{1}^{2} s_{7}^{2} s_{9}^{5}-8 s_{1} s_{2} s_{4} s_{8} s_{9}^{5}+4 s_{1}^{2} s_{4} s_{9}^{6}\right) \text {. }
\end{aligned}
$$


This yields the sought for cubic on the right hand side of (2.1). Finally upon the variable transformation $\tau=\frac{x}{c}$ the quadric B.8 reads, after multiplying by $c^{2} \tau^{4}$

$$
y^{2} \equiv\left[\frac{1}{c} x^{2}\left(2 \phi_{3}(t) u^{\prime}+\phi_{2}(t) w\right)\right]^{2}=x^{3}+d x^{2}+e c x+k c^{2} .
$$

This describes the double cover over $\mathbb{P}^{1}$ with coordinate $y$, that finally brings $(\mathrm{B} .11)$ in the Tate form (2.1). In the concrete calculation we obtain the coefficients $c, d, e$ as well as the Tate coefficients $a_{i}$ in (2.1) as

$$
\begin{aligned}
a_{2}= & -s_{6}^{2}+4 s_{5} s_{7}-8 s_{3} s_{8}+\frac{12 s_{4} s_{8}^{2}}{s_{9}}+4 s_{2} s_{9}, \\
a_{4}= & -\frac{1}{s_{9}^{2}} 8\left(6 s_{4}^{2} s_{8}^{4}-s_{4} s_{9}\left(s_{6}^{2} s_{8}^{2}-4 s_{5} s_{7} s_{8}^{2}+8 s_{3} s_{8}^{3}+s_{5} s_{6} s_{8} s_{9}-6 s_{2} s_{8}^{2} s_{9}-2 s_{5}^{2} s_{9}^{2}+6 s_{1} s_{8} s_{9}^{2}\right)\right. \\
& +s_{9}^{2}\left(2 s_{3}^{2} s_{8}^{2}+s_{7}\left(-s_{2} s_{6} s_{8}+2 s_{1} s_{7} s_{8}+2 s_{2} s_{5} s_{9}-s_{1} s_{6} s_{9}\right)\right. \\
& \left.\left.+s_{3}\left(s_{6}^{2} s_{8}-2 s_{5} s_{7} s_{8}-s_{5} s_{6} s_{9}-2 s_{2} s_{8} s_{9}+2 s_{1} s_{9}^{2}\right)\right)\right) \\
& \frac{1}{a_{6}} 16\left(4 s_{4}^{3} s_{8}^{6}-s_{9}^{3}\left(s_{7}\left(s_{2} s_{8}-s_{1} s_{9}\right)+s_{3}\left(-s_{6} s_{8}+s_{5} s_{9}\right)\right)^{2}\right. \\
& s_{9}^{3} \\
& +s_{4}^{2} s_{8}^{2} s_{9}\left(s_{6}^{2} s_{8}^{2}-4 s_{5} s_{7} s_{8}^{2}+2 s_{5} s_{6} s_{8} s_{9}-3 s_{5}^{2} s_{9}^{2}+8 s_{8}\left(s_{3} s_{8}^{2}-s_{2} s_{8} s_{9}+s_{1} s_{9}^{2}\right)\right) \\
& +2 s_{4} s_{9}^{2}\left(2 s_{3}^{2} s_{8}^{4}+2 s_{2}^{2} s_{8}^{2} s_{9}^{2}+s_{3} s_{8}\left(s_{6}^{2} s_{8}^{2}-2 s_{5} s_{7} s_{8}^{2}-s_{5}^{2} s_{9}^{2}+4 s_{8} s_{9}\left(-s_{2} s_{8}+s_{1} s_{9}\right)\right)\right. \\
& +s_{1}\left(2 s_{7}^{2} s_{8}^{3}+s_{7} s_{8} s_{9}\left(-3 s_{6} s_{8}+s_{5} s_{9}\right)+2 s_{9}^{2}\left(s_{6}^{2} s_{8}-s_{5} s_{6} s_{9}+s_{1} s_{9}^{2}\right)\right) \\
& \left.\left.+s_{2}\left(3 s_{5} s_{7} s_{8}^{2} s_{9}+2 s_{5}^{2} s_{9}^{3}-4 s_{1} s_{8} s_{9}^{3}-s_{6}\left(s_{7} s_{8}^{3}+2 s_{5} s_{8} s_{9}^{2}\right)\right)\right)\right)
\end{aligned}
$$

with all $a_{i}=0$ for odd $i=1,3$.

The birational coordinate transformation from $(u, v)$ to $(x, y)$ can be determined by going backwards through the relations (B.11) and (B.5) using

$$
t=-\frac{s_{8}}{s_{9}}+\frac{c}{x}=\frac{v^{\prime}}{u^{\prime}}
$$

We readily obtain from this and the identification of $y$ in (B.11)

$$
\begin{aligned}
& x=c \frac{u+s_{9} \frac{e_{2}}{e_{3}} w}{v+\frac{s_{8}}{s_{9}} u} \\
& y=c \frac{u^{\prime 2}\left(2 \phi_{3}(t) u^{\prime}+\phi_{2}(t) w\right)}{\left(v+\frac{s_{8}}{s_{9}} u\right)^{2}}=\left.c \frac{-f_{2}\left(u^{\prime}, v^{\prime}\right) w-2 f_{1}\left(u^{\prime}, v^{\prime}\right) w^{2}}{\left(v+\frac{s_{8}}{s_{9}} u\right)^{2}}\right|_{u^{\prime}=u+s_{9} \frac{e_{2}}{e_{3}}, v^{\prime}=v-s_{8} \frac{e_{2}}{e_{3}}}
\end{aligned}
$$


with $f_{i}$ defined in (B.6). Here we have used the elementary relation $2 f_{3}+f_{2}=-f_{2}-2 f_{1}$ that directly follows from (B.6) to rewrite the numerator of $y$. In the case at hand we have

$$
e_{2}=s_{7} s_{8}^{2}-s_{6} s_{8} s_{9}+s_{5} s_{9}^{2}, \quad e_{3}=-s_{4} s_{8}^{3}+s_{3} s_{8}^{2} s_{9}-s_{2} s_{8} s_{9}^{2}+s_{1} s_{9}^{3} .
$$

This implies

$$
\begin{aligned}
& x=-\frac{4\left(s_{9}\left(s_{7} s_{8}^{2}+s_{9}\left(-s_{6} s_{8}+s_{5} s_{9}\right)\right) w+\left(-s_{4} s_{8}^{3}+s_{9}\left(s_{3} s_{8}^{2}-s_{2} s_{8} s_{9}+s_{1} s_{9}^{2}\right)\right) u\right)}{s_{9}\left(u s_{8}+v s_{9}\right)} . \\
& y=-4 w \frac{m_{1} u^{2}+m_{2} u v+m_{3} v^{2}+m_{4} u w+m_{5} v w}{\left(u s_{8}+v s_{9}\right)^{2}}
\end{aligned}
$$

where the coefficients $m_{i}$ are given by

$$
\begin{aligned}
& m_{1}=s_{4} s_{5} s_{8}^{3}+s_{2} s_{8}^{2}\left(-s_{7} s_{8}+s_{6} s_{9}\right)+s_{9}\left(-s_{3} s_{5} s_{8}^{2}+s_{1}\left(3 s_{7} s_{8}^{2}-3 s_{6} s_{8} s_{9}+2 s_{5} s_{9}^{2}\right)\right), \\
& m_{2}=s_{4} s_{6} s_{8}^{3}+s_{3} s_{8}\left(-2 s_{7} s_{8}^{2}+s_{9}\left(s_{6} s_{8}-2 s_{5} s_{9}\right)\right)+s_{9}\left(-s_{1} s_{6} s_{9}^{2}+s_{2}\left(2 s_{7} s_{8}^{2}-s_{6} s_{8} s_{9}+2 s_{5} s_{9}^{2}\right)\right), \\
& m_{3}=-\left(s_{9}^{2}\left(s_{7}\left(-s_{2} s_{8}+s_{1} s_{9}\right)+s_{3}\left(s_{6} s_{8}-s_{5} s_{9}\right)\right)+s_{4} s_{8}\left(2 s_{7} s_{8}^{2}+3 s_{9}\left(-s_{6} s_{8}+s_{5} s_{9}\right)\right)\right), \\
& m_{4}=s_{6}^{2} s_{8}^{2} s_{9}-s_{6}\left(s_{7} s_{8}^{3}+3 s_{5} s_{8} s_{9}^{2}\right)+2\left(s_{4} s_{8}^{4}+s_{9}\left(s_{5} s_{7} s_{8}^{2}-s_{3} s_{8}^{3}+s_{2} s_{8}^{2} s_{9}+s_{5}^{2} s_{9}^{2}-s_{1} s_{8} s_{9}^{2}\right)\right), \\
& m_{5}=-2 s_{7}^{2} s_{8}^{3}+s_{7} s_{8} s_{9}\left(3 s_{6} s_{8}-2 s_{5} s_{9}\right)+s_{9}\left(2 s_{4} s_{8}^{3}+s_{9}\left(-s_{6}^{2} s_{8}-2 s_{3} s_{8}^{2}+s_{5} s_{6} s_{9}+2 s_{2} s_{8} s_{9}-2 s_{1} s_{9}^{2}\right)\right) .
\end{aligned}
$$

We readily obtain the inverse variable transformation determining $u$ and $v$ in terms of $x$, $y$ by inverting (B.16). Equivalently, one can directly invert the relation (B.11) to obtain

$$
u_{1 / 2}^{\prime}=\left.\frac{-x^{3} \phi_{2}(t) \pm c x y}{2 x^{3} \phi_{3}(t)}\right|_{t=-\frac{s_{8}}{s_{9}}+\frac{c}{x}}=-\left.\frac{2 \phi_{1}(t) x^{2}}{\phi_{2}(t) x^{2} \pm c y}\right|_{t=-\frac{s_{8}}{s_{9}}+\frac{c}{x}}, \quad v^{\prime}=u^{\prime}\left(-\frac{s_{8}}{s_{9}}+\frac{c}{x}\right) .
$$

Here we have made us of the equation (B.6) to arrive at the second equality for $u^{\prime}$ and inverted (B.13) to obtain $v^{\prime}$. In order to simply the expressions on the right side we have to use the Weierstrass equation (B.11) and we obtain for $u$ and $v$, employing (B.5),

$$
u=2 s_{9} \frac{q_{1} x+q_{2} y+q_{3}}{r_{1} x^{2}+r_{2} x+r_{3} y+r_{4}}, \quad v=2 s_{9} \frac{n_{1} x+n_{2} y+n_{3}}{p_{1} x^{2}+p_{2} x+p_{3} y+p_{4}},
$$

where the explicit form of $q_{i}, r_{j}, n_{k}$ and $p_{l}$ follow from (B.19) and are provided upon request. 


\section{References}

[1] R. Donagi and M. Wijnholt, "Model Building with F-Theory," arXiv:0802.2969 [hep-th].

[2] C. Beasley, J. J. Heckman, and C. Vafa, "GUTs and Exceptional Branes in F-theory - I," JHEP 01 (2009) 058, arXiv:0802.3391 [hep-th].

[3] C. Beasley, J. J. Heckman, and C. Vafa, "GUTs and Exceptional Branes in F-theory - II: Experimental Predictions," JHEP 01 (2009) 059, arXiv:0806.0102 [hep-th].

[4] R. Donagi and M. Wijnholt, "Breaking GUT Groups in F-Theory," Adv.Theor.Math.Phys. 15 (2011) 1523-1604, arXiv:0808.2223 [hep-th].

[5] R. Blumenhagen, T. W. Grimm, B. Jurke, and T. Weigand, "Global F-theory GUTs," Nucl.Phys. B829 (2010) 325-369, arXiv:0908.1784 [hep-th].

[6] J. Marsano, N. Saulina, and S. Schafer-Nameki, "Compact F-theory GUTs with U(1) (PQ)," JHEP 1004 (2010) 095, arXiv:0912.0272 [hep-th].

[7] C.-M. Chen, J. Knapp, M. Kreuzer, and C. Mayrhofer, "Global SO(10) F-theory GUTs," JHEP 1010 (2010) 057, arXiv:1005.5735 [hep-th].

[8] T. W. Grimm, S. Krause, and T. Weigand, "F-Theory GUT Vacua on Compact Calabi-Yau Fourfolds," JHEP 1007 (2010) 037, arXiv:0912.3524 [hep-th].

[9] J. Knapp and M. Kreuzer, "Toric Methods in F-theory Model Building," Adv.High Energy Phys. 2011 (2011) 513436, arXiv:1103.3358 [hep-th].

[10] J. J. Heckman, "Particle Physics Implications of F-theory," Ann.Rev.Nucl.Part.Sci. 60 (2010) 237-265, arXiv:1001.0577 [hep-th].

[11] T. Weigand, "Lectures on F-theory compactifications and model building," Class.Quant.Grav. 27 (2010) 214004, arXiv:1009.3497 [hep-th].

[12] A. Maharana and E. Palti, "Models of Particle Physics from Type IIB String Theory and F-theory: A Review," arXiv:1212.0555 [hep-th].

[13] C. Vafa, "Evidence for F theory," Nucl.Phys. B469 (1996) 403-418, arXiv:hep-th/9602022 [hep-th]. 
[14] D. R. Morrison and C. Vafa, "Compactifications of F theory on Calabi-Yau threefolds. 1," Nucl.Phys. B473 (1996) 74-92, arXiv:hep-th/9602114 [hep-th].

[15] D. R. Morrison and C. Vafa, "Compactifications of F theory on Calabi-Yau threefolds. 2.," Nucl.Phys. B476 (1996) 437-469, arXiv: hep-th/9603161 [hep-th].

[16] K. Kodaira, "On compact analytic surfaces: Ii," The Annals of Mathematics $\mathbf{7 7}$ no. 3, (1963) 563-626.

[17] J. Tate, "Algorithm for determining the type of a singular fiber in an elliptic pencil," Modular functions of one variable IV (1975) 33-52.

[18] M. Bershadsky, K. A. Intriligator, S. Kachru, D. R. Morrison, V. Sadov, et al., "Geometric singularities and enhanced gauge symmetries," Nucl.Phys. B481 (1996) 215-252, arXiv:hep-th/9605200 [hep-th].

[19] M. Esole and S.-T. Yau, "Small resolutions of SU(5)-models in F-theory," arXiv:1107.0733 [hep-th].

[20] J. Marsano and S. Schafer-Nameki, "Yukawas, G-flux, and Spectral Covers from Resolved Calabi-Yau's," JHEP 1111 (2011) 098, arXiv:1108.1794 [hep-th].

[21] C. Lawrie and S. Schafer-Nameki, "The Tate Form on Steroids: Resolution and Higher Codimension Fibers," arXiv:1212.2949 [hep-th].

[22] P. Candelas and A. Font, "Duality between the webs of heterotic and type II vacua," Nucl.Phys. B511 (1998) 295-325, arXiv:hep-th/9603170 [hep-th].

[23] P. Candelas, E. Perevalov, and G. Rajesh, "Toric geometry and enhanced gauge symmetry of F theory / heterotic vacua," Nucl.Phys. B507 (1997) 445-474, arXiv:hep-th/9704097 [hep-th].

[24] V. Braun, "Toric Elliptic Fibrations and F-Theory Compactifications," JHEP 1301 (2013) 016, arXiv:1110.4883 [hep-th].

[25] R. Donagi and M. Wijnholt, "Higgs Bundles and UV Completion in F-Theory," arXiv:0904.1218 [hep-th]. 
[26] J. Marsano, N. Saulina, and S. Schafer-Nameki, "Monodromies, Fluxes, and Compact Three-Generation F-theory GUTs," JHEP 0908 (2009) 046, arXiv:0906.4672 [hep-th].

[27] E. Dudas and E. Palti, "Froggatt-Nielsen models from E(8) in F-theory GUTs," JHEP 1001 (2010) 127, arXiv:0912.0853 [hep-th].

[28] M. Cvetic, I. Garcia-Etxebarria, and J. Halverson, "Global F-theory Models: Instantons and Gauge Dynamics," JHEP 1101 (2011) 073, arXiv:1003.5337 [hep-th].

[29] E. Dudas and E. Palti, "On hypercharge flux and exotics in F-theory GUTs," JHEP 1009 (2010) 013, arXiv:1007.1297 [hep-ph].

[30] M. J. Dolan, J. Marsano, N. Saulina, and S. Schafer-Nameki, "F-theory GUTs with U(1) Symmetries: Generalities and Survey," Phys.Rev. D84 (2011) 066008, arXiv:1102.0290 [hep-th].

[31] J. Marsano, H. Clemens, T. Pantev, S. Raby, and H.-H. Tseng, "A Global SU(5) F-theory model with Wilson line breaking," JHEP 1301 (2013) 150, arXiv:1206.6132 [hep-th].

[32] A. Néron, "Modeles minimaux des variétés abéliennes sur les corps locaux et globaux," Publications Mathématiques de L'IHÉS 21 no. 1, (1964) 5-125.

[33] T. Shioda, "Mordell-Weil lattices and Galois representation. I," Proc. Japan Acad. 65A (1989) 268-271.

[34] T. Shioda, "On the Mordell-Weil lattices," Comment. Math. Univ. St. Paul 39 no. 2, (1990) 211-240.

[35] R. Wazir, "Arithmetic on Elliptic Threefolds," arXiv:math/0112259 [math.NT].

[36] J. H. Silverman, The arithmetic of elliptic curves, vol. 106. Springer, 2009.

[37] P. S. Aspinwall and D. R. Morrison, "Nonsimply connected gauge groups and rational points on elliptic curves," JHEP 9807 (1998) 012, arXiv:hep-th/9805206 [hep-th]. 
[38] P. S. Aspinwall, S. H. Katz, and D. R. Morrison, "Lie groups, Calabi-Yau threefolds, and F theory," Adv.Theor.Math.Phys. 4 (2000) 95-126, arXiv:hep-th/0002012 [hep-th].

[39] T. W. Grimm and T. Weigand, "On Abelian Gauge Symmetries and Proton Decay in Global F-theory GUTs," Phys.Rev. D82 (2010) 086009, arXiv:1006.0226 [hep-th].

[40] A. P. Braun, A. Collinucci, and R. Valandro, "G-flux in F-theory and algebraic cycles," Nucl.Phys. B856 (2012) 129-179, arXiv:1107.5337 [hep-th].

[41] S. Krause, C. Mayrhofer, and T. Weigand, " $G_{4}$ flux, chiral matter and singularity resolution in F-theory compactifications," Nucl.Phys. B858 (2012) 1-47, arXiv:1109.3454 [hep-th].

[42] T. W. Grimm and H. Hayashi, "F-theory fluxes, Chirality and Chern-Simons theories," JHEP 1203 (2012) 027, arXiv:1111.1232 [hep-th].

[43] D. R. Morrison and D. S. Park, "F-Theory and the Mordell-Weil Group of Elliptically-Fibered Calabi-Yau Threefolds," JHEP 1210 (2012) 128, arXiv:1208.2695 [hep-th].

[44] M. Cvetic, T. W. Grimm, and D. Klevers, "Anomaly Cancellation And Abelian Gauge Symmetries In F-theory," JHEP 1302 (2013) 101, arXiv:1210.6034 [hep-th].

[45] C. Mayrhofer, E. Palti, and T. Weigand, "U(1) symmetries in F-theory GUTs with multiple sections," arXiv:1211.6742 [hep-th].

[46] V. Braun, T. W. Grimm, and J. Keitel, "New Global F-theory GUTs with U(1) symmetries," arXiv:1302.1854 [hep-th].

[47] H. Hayashi, T. Kawano, Y. Tsuchiya, and T. Watari, "More on Dimension-4 Proton Decay Problem in F-theory - Spectral Surface, Discriminant Locus and Monodromy," Nucl.Phys. B840 (2010) 304-348, arXiv:1004.3870 [hep-th].

[48] J. Marsano, "Hypercharge Flux, Exotics, and Anomaly Cancellation in F-theory GUTs," Phys.Rev.Lett. 106 (2011) 081601, arXiv:1011.2212 [hep-th].

[49] A. Grassi and V. Perduca, "Weierstrass models of elliptic toric K3 hypersurfaces and symplectic cuts," arXiv:1201.0930 [math.AG]. 
[50] V. V. Batyrev, "Dual polyhedra and mirror symmetry for Calabi-Yau hypersurfaces in toric varieties," J.Alg.Geom. 3 (1994) 493-545, arXiv:alg-geom/9310003 [alg-geom].

[51] A. Klemm, P. Mayr, and C. Vafa, "BPS states of exceptional noncritical strings," arXiv:hep-th/9607139 [hep-th].

[52] E. P. J. Borchmann, C. Mayrhofer and T. Weigand, "Elliptic fibrations for SU(5) x U(1) x U(1) F-theory vacua," arXiv:1303.5054 [hep-th].

[53] S. Katz, D. R. Morrison, S. Schafer-Nameki, and J. Sully, "Tate's algorithm and F-theory," JHEP 1108 (2011) 094, arXiv:1106.3854 [hep-th].

[54] T. W. Grimm, "The N=1 effective action of F-theory compactifications," Nucl.Phys. B845 (2011) 48-92, arXiv:1008.4133 [hep-th].

[55] D. S. Park, "Anomaly Equations and Intersection Theory," JHEP 1201 (2012) 093, arXiv:1111.2351 [hep-th].

[56] T. W. Grimm and R. Savelli, "Gravitational Instantons and Fluxes from M/F-theory on Calabi-Yau fourfolds," Phys.Rev. D85 (2012) 026003, arXiv:1109.3191 [hep-th].

[57] F. Bonetti and T. W. Grimm, "Six-dimensional $(1,0)$ effective action of F-theory via M-theory on Calabi-Yau threefolds," JHEP 1205 (2012) 019, arXiv:1112.1082 [hep-th].

[58] P. Griffiths and J. Harris, Principles of algebraic geometry, vol. 52. Wiley-interscience, 2011.

[59] J. Erler, "Anomaly cancellation in six-dimensions," J.Math.Phys. 35 (1994) 1819-1833, arXiv:hep-th/9304104 [hep-th].

[60] G. Honecker, "Massive U(1)s and heterotic five-branes on K3," Nucl.Phys. B748 (2006) 126-148, arXiv:hep-th/0602101 [hep-th].

[61] V. Sadov, "Generalized Green-Schwarz mechanism in F theory," Phys.Lett. B388 (1996) 45-50, arXiv:hep-th/9606008 [hep-th].

[62] A. Grassi and D. R. Morrison, "Anomalies and the Euler characteristic of elliptic Calabi-Yau threefolds," arXiv:1109.0042 [hep-th]. 
[63] A. Klemm, B. Lian, S. Roan, and S.-T. Yau, "Calabi-Yau fourfolds for M theory and F theory compactifications," Nucl.Phys. B518 (1998) 515-574, arXiv:hep-th/9701023 [hep-th].

[64] M. Cvetič, A. Grassi, D. Klevers, and H. Piragua, "Chiral Four-Dimensional F-Theory Compactifications With SU(5) and Multiple U(1)-Factors," arXiv:1306.3987 [hep-th].

[65] I. Connell, "Elliptic curve handbook," Preprint (1996) . 